\title{
PARABOLIC QUASI-VARIATIONAL INEQUALITIES WITH GRADIENT-TYPE CONSTRAINTS*
}

\author{
MICHAEL HINTERMÜLLER ${ }^{\dagger}$ AND CARLOS N. RAUTENBERG ${ }^{\ddagger}$
}

\begin{abstract}
This paper considers a class of nonlinear evolution quasi-variational inequality (QVI) problems with pointwise gradient constraints in vector-valued function spaces. The existence and approximation of solutions is addressed based on a combination of $C_{0}$-semigroup methods, Mosco convergence, and monotone operator techniques developed by Brézis. An algorithm based on semidiscretization in time is proposed and its numerical implementation based on a penalty approach and semismooth Newton methods is studied. This paper ends with a report on numerical tests which involve the $p$-Laplacian and several types of nonlinear constraints.
\end{abstract}

Key words. evolution quasi-variational inequality, gradient constraints, semismooth Newton method

AMS subject classifications. 35K86, 47J20, 49J40, 49M15, 65J15, 65K10

DOI. $10.1137 / 120874308$

1. Introduction. Variational inequalities (VIs) were first introduced in the context of linear elastostatics in the form of the Signorini problem by Fichera in [14]. In the mathematical literature, however, VIs were introduced by Lions and Stampacchia in [28], who established the first abstract results on existence, uniqueness, and continuity with respect to parameters. A systematic approach to time-dependent problems based on pseudomonotone operators and $C_{0}$-semigroups to describe derivatives with respect to the time variable was established by Brézis in [7], and in [25] an account of this approach with generalizations and several applications is found.

Quasi-variational inequalities (QVIs), of both elliptic and parabolic type, respectively, were introduced by Bensoussan and Lions in [26] and [4]. This problem class arises in the mathematical formulation of diverse phenomena in the applied sciences such as game theory, superconductivity, and solid or continuum mechanics; see, for example, $[5,12,16,24,27,30,32]$ and the monographs [3, 23] as well as the references therein. Although methods of approximation have been developed for QVIs when the constraint set is determined by pointwise bounds on the state variable, the literature is rather scarce (exceptions to this are $[24,35,36]$ ) when constraint sets are related to (pointwise) bounds on the gradient of the state variable.

In this paper we study a general abstract version of a parabolic QVI which is a generalization of the VIs described in [25] and where the class of constraint sets that involve nonconstant (in time and space) bounds on the norm of the gradient is included. We establish a novel approximation theorem which is suitable for the analysis

*Received by the editors April 20, 2012; accepted for publication (in revised form) June 4, 2013; published electronically October 24, 2013. This work was supported by the Austrian Ministry of Science and Education and the Austrian Science Fund FWF through START-Project Y305 "Interfaces and Free Boundaries," the German Research Foundation DFG Research Center MATHEON through Projects C28 and C31, and DFG Priority Program SPP 1253 through subproject "Elliptic Mathematical Programs with Equilibrium Constraints (MPECs) in Function Space: Optimality Conditions and Numerical Realization."

http://www.siam.org/journals/siopt/23-4/87430.html

${ }^{\dagger}$ Department of Mathematics, Humboldt-University of Berlin, 10099 Berlin, Germany (hint@ math.hu-berlin.de).

${ }^{\ddagger}$ Department of Mathematics and Scientific Computing, Karl-Franzens-University of Graz, A-8010 Graz, Austria (carlos.rautenberg@uni-graz.at).

2090 
of our numerical approximation scheme, and it also allows us to prove existence of a solution to the parabolic QVI problem. Our approach is based on a combination of the approximation methods developed in $C_{0}$-semigroup theory (mainly for the analytical forms of the Trotter-Kato theorem), the techniques based on monotonicity developed by Brézis in [7], and developments related to Mosco convergence for closed, convex sets. The approximate problems (semidiscrete in time) reduce to sequences of a finite number of elliptic QVIs of the type studied in [19]. Numerically, a solution to these problems is obtained by a fixed-point iteration that involves the resolution of penalized subproblems by a semismooth Newton solver. For discussing the practical behavior of our algorithm, we provide examples involving time realizations of the $p$-Laplace operator and several types of gradient constraints.

The rest of this paper is organized as follows. In section 2 we state the class of parabolic QVI problems under investigation and provide the most important examples. In section 3 we establish the necessary structure for the approximate spaces and a type of convergence (called $\mathbf{\Lambda}$-convergence) that is used in Theorem 1, which constitutes the main result of this paper. Conditions on the approximate set-valued constraint mappings and infinitesimal generators are given in section 3.1. Subsequently, section 4 is devoted to studying the regularity (in time) of the solutions to the parabolic QVI problem. The main result is established in Theorem 2, and examples of applications are given in section 4.1. In section 5 we provide a concrete characterization of the semigroup of interest, the set-valued maps that determine the constraints in the case when these are given by gradient-type constraints, and an approach to initial value problems. The algorithm is studied in section 6 where a crucial result of strong convergence of the approximate infinitesimal generators is established in Proposition 6.1. Finally, in section 7 we report on numerical tests and details of the implementation of our algorithm.

1.1. Notation. Throughout this paper, for a Banach space $X$ its norm is written as $|\cdot|_{X}$, and for $f \in X^{\prime}$ (the topological dual of $X$ ) we denote $f(x):=(f, x)_{X}$ for $x \in X$. If $H$ is a Hilbert space, we identify its dual $H^{\prime}$ with $H$, and denote the duality pairing as $\langle f, x\rangle_{H}$ for $f \in H^{\prime}$ and $x \in H$. We denote by $\mathscr{L}(V, W)$ the vector space of linear bounded operators between the normed vector spaces $V$ and $W$ with norm $|G|_{\mathscr{L}(V, W)}=\sup _{|v|_{V=1}}|G v|_{W}$ for $G \in \mathscr{L}(V, W)$.

The natural numbers, integers, real, and complex numbers are denoted by $\mathbb{N}, \mathbb{Z}$, $\mathbb{R}$, and $\mathbb{C}$, respectively, and by $\mathbb{R}^{+}$we denote the set of positive real numbers and $\mathbb{R}_{0}^{+}=\mathbb{R}^{+} \cup\{0\}$.

For $v_{0} \in X$ and $R>0$ we write $B_{R}\left(v_{0}\right):=\left\{v \in X:\left|v-v_{0}\right|_{X}<R\right\}$ (or $\left.B_{R}\left(v_{0} ; X\right)\right)$ and its closure in $X$ is written as $\bar{B}_{R}\left(v_{0}\right)$ (or $\bar{B}_{R}\left(v_{0} ; X\right)$ ). We denote the strong convergence of a sequence $\left\{u_{n}\right\} \subset X$ to $u \in X$ by $u_{n} \rightarrow u$. Weak convergence is written as $u_{n} \rightarrow u$. The Lebesgue measure of a measurable set $\Omega$ is denoted by $|\Omega|$, and we say that a property holds "almost everywhere (a.e.) in $\Omega$ " if it is true in $\Omega$ except for a subset $\Omega_{0} \subset \Omega$ such that $\left|\Omega_{0}\right|=0$. For a real-valued function $v$, we define $v^{+}=\max (0, v)$ in the pointwise sense, that is, for $x \in \Omega, v^{+}(x)=v(x)$ if $v(x) \geq 0$ and $v^{+}(x)=0$ otherwise.

Let $\Omega \subset \mathbb{R}^{N}$ with $N \geq 2$ be a bounded, open domain with Lipschitz boundary $\partial \Omega$. We write $L^{p}(\Omega)$ (or $L^{p}(\Omega ; \mathbb{R})$ ), with $1 \leq p \leq \infty$ for the usual Lebesgue spaces of real-valued functions, and $L_{\nu}^{\infty}(\Omega):=\left\{v \in L^{\infty}(\Omega): v(\mathbf{x}) \geq \nu>0\right.$ a.e. $\left.\mathbf{x} \in \Omega\right\}$. We say that $p^{\prime}$ is the Hölder conjugate of $p$ if $\frac{1}{p}+\frac{1}{p^{\prime}}=1$. We denote by $W_{0}^{1, p}(\Omega)$ for $1<p<\infty$ the Sobolev space of weakly differentiable functions in $L^{p}(\Omega)$ with zero value at the boundary $\partial \Omega$ (in the sense of the trace), whose weak derivatives also 
belong to $L^{p}(\Omega)$ (see [1] for a definition of the Sobolev space), endowed with norm $|v|_{W_{0}^{1, p}(\Omega)}=\left(\int_{\Omega}|\nabla v(\mathbf{x})|_{\mathbb{R}^{N}}^{p} \mathrm{~d} \mathbf{x}\right)^{1 / p}$; we also denote $H_{0}^{1}(\Omega):=W_{0}^{1,2}(\Omega)$.

Let $\mathrm{I}=(0, T)$ with $0<T \leq \infty$ and let $X$ be a Banach space. Then, a function $f: \mathrm{I} \rightarrow X$ is Bochner measurable if there is a sequence $\left\{f_{n}\right\}$ of simple $X$-valued functions such that $\lim _{n \rightarrow \infty} f_{n}(t)=f(t)$ a.e. in I (see [17]). We denote by $L^{p}(\mathrm{I} ; X)$ the (Lebesgue-Bochner) space of Bochner measurable $X$-valued mappings with domain I such that $\int_{\mathrm{I}}|f(t)|_{X}^{p} \mathrm{~d} t<\infty$ and the integral is taken in the Lebesgue sense.

Since we will deal with convergence of closed and convex subsets of reflexive Banach spaces, we make use of Mosco convergence (see [29, 34]).

Definition 1.1 (Mosco convergence). Let $K$ and $K_{n}$, for each $n \in \mathbb{N}$, be nonempty, closed, and convex subsets of $X$, a reflexive Banach space. We say that the sequence $\left\{K_{n}\right\}$ converges to $K$ in the Mosco sense (and denote this by $K_{n} \rightarrow K$ ) if:

i. $\forall v \in K, \exists v_{n} \in K_{n}: v_{n} \rightarrow v$ in $X$.

ii. If $v_{n} \in K_{n}$ and $v_{n} \rightarrow v$ in $X$ with $n \in \hat{\mathbb{N}} \subset \mathbb{N}$, then $v \in K$.

2. Problem formulation. Let $\mathscr{V}$ be a reflexive separable Banach space, let $\mathscr{V}^{\prime}$ be its topological dual, and let $\mathscr{H}$ be a separable Hilbert space. Suppose $\left(\mathscr{V}, \mathscr{H}, \mathscr{V}^{\prime}\right)$ is a Gelfand triple; that is, we have the embeddings $\mathscr{V} \hookrightarrow \mathscr{H} \hookrightarrow \mathscr{V}^{\prime}$, where we identify $\mathscr{H}$ with its dual $\mathscr{H}^{\prime}$ and the embedding $\mathscr{V} \hookrightarrow \mathscr{H}$ is dense and continuous (and hence also is $\mathscr{H} \hookrightarrow \mathscr{V}^{\prime}$; see [10]). For $f \in \mathscr{V}^{\prime}$ and $v \in \mathscr{V}$ the duality pairing $(f, v)_{\mathscr{V}}$ is supposed to be the continuous extension of $\langle\cdot, \cdot\rangle_{\mathscr{H}}$ on $\mathscr{H} \times \mathscr{V}$, so that there is a sequence $\left\{h_{n}\right\} \subset \mathscr{H}$ for which $(f, v)_{\mathscr{V}}=\lim _{n \rightarrow \infty}\left\langle h_{n}, v\right\rangle_{\mathscr{H}}$ uniformly on bounded sets of $\mathscr{V}$.

Suppose $\left(V, H, V^{\prime}\right)$ denotes a Gelfand triple (with $V$ a separable, reflexive Banach space and $H$ a separable Hilbert space). Then, for $p \geq 2$ and $\mathrm{I}=(0, T)$ with $0<T<\infty,\left(\mathscr{V}, \mathscr{H}, \mathscr{V}^{\prime}\right)$ is also a Gelfand triple, where $\mathscr{V}=L^{p}(\mathrm{I} ; V)$ and $\mathscr{H}=$ $L^{2}(\mathrm{I} ; H)$. In this case, $\mathscr{V}^{\prime}=L^{p^{\prime}}\left(\mathrm{I} ; V^{\prime}\right)$ since I is $\sigma$-finite (see Theorem 1.5, Chapter III in [37]) and with $p^{\prime}$ the Hölder conjugate of $p$. If $T=\infty$, then the previous holds for $p=2$. These are the cases most frequently used in what follows. A typical example for $\left(V, H, V^{\prime}\right)$ is given by $\left(H_{0}^{1}(\Omega), L^{2}(\Omega), H^{-1}(\Omega)\right)$.

We assume that the (usually nonlinear) map $\mathcal{A}: \mathscr{V} \rightarrow \mathscr{V}^{\prime}$ is as follows:

H1. uniformly monotone, i.e., there are constants $c>0$ and $r>1$ such that,

$$
(\mathcal{A}(u)-\mathcal{A}(v), u-v)_{\mathscr{V}} \geq c|u-v|_{\mathscr{V}}^{r} \quad \forall u, v \in \mathscr{V}
$$

H2. hemicontinuous, i.e., the real-valued function $\zeta \mapsto(\mathcal{A}(u+\zeta v), w)_{\mathscr{V}}$ is continuous for $\zeta \in[0,1]$ for all $u, v, w \in \mathscr{V}$;

H3. bounded, i.e., it maps bounded sets in $\mathscr{V}$ into bounded sets in $\mathscr{V}^{\prime}$.

Since $\mathscr{V}$ is assumed to be reflexive, then $\mathrm{H} 1$ together with $\mathrm{H} 2$ imply that $\mathcal{A}$ is pseudomonotone, i.e., if $u_{n} \rightarrow u$ and $\varlimsup_{n \rightarrow \infty}\left(\mathcal{A}\left(u_{n}\right), u_{n}-u\right)_{\mathscr{V}} \leq 0$, then $(\mathcal{A}(u), u-$ $v)_{\mathscr{V}} \leq \underline{\lim }_{n \rightarrow \infty}\left(\mathcal{A}\left(u_{n}\right), u_{n}-v\right)_{\mathscr{V}}$ for all $v \in \mathscr{V}$. If also $\mathrm{H} 3$ is assumed or $\mathscr{V}$ is a real Banach space (see Proposition 26.4 in [38]), then $\mathcal{A}$ is demicontinuous, i.e., if $u_{n} \rightarrow u$ in $\mathscr{V}$, then $\mathcal{A}\left(u_{n}\right) \rightarrow \mathcal{A}(u)$ in the weak-star topology and hence $\mathcal{A}\left(u_{n}\right) \rightarrow \mathcal{A}(u)$ in $\mathscr{V}^{\prime}$ (due to the reflexivity of $\mathscr{V}$ ).

Mappings $\mathcal{A}: \mathscr{V} \rightarrow \mathscr{V}^{\prime}$ that satisfy $\mathrm{H} 1, \mathrm{H} 2$, and $\mathrm{H} 3$ are very common in applications. For example, they can be obtained as time realizations on $\mathscr{V}=L^{p}(\mathrm{I} ; V)$ of operators $A: V \rightarrow V^{\prime}$ that satisfy $\mathrm{H} 1, \mathrm{H} 2$, and (a slightly stronger condition than) $\mathrm{H} 3$ over $V$. By time realization we mean $\mathcal{A}(w)(t)=A(w(t))$ for all $w \in L^{p}(\mathrm{I} ; V)$. This is shown in the following proposition (a similar but less general result can be found in Lemma 4.1, Chapter III in [37]). 
Proposition 2.1. Let $\mathscr{V}=L^{p}(\mathrm{I} ; V)$ for $p \geq 2$ with $\mathrm{I}=(0, T)$ and $0<T \leq \infty$, and let $A: V \rightarrow V^{\prime}$ (with $V$ some reflexive separable Banach space) satisfy $\mathrm{H} 1$ with $r=p$, H2 over $V$, and $|A(v)|_{V^{\prime}} \leq C|v|_{V}^{p-1}$ for all $v \in V$. Then, the map $\mathcal{A}: \mathscr{V} \rightarrow \mathscr{V}^{\prime}$, defined as

$$
(\mathcal{A}(v), w)_{\mathscr{V}}=\int_{\mathrm{I}}(A(v(t)), w(t))_{V} \mathrm{~d} t \quad \forall v, w \in \mathscr{V},
$$

satisfies $\mathrm{H} 1, \mathrm{H} 2$, and $\mathrm{H} 3$.

Proof. Since $A$ is monotone, hemicontinuous, and bounded, it is demicontinuous; the reflexivity of $V$ implies that if $v_{n} \rightarrow v$ in $V$, then $A\left(v_{n}\right) \rightarrow A(v)$ in $V^{\prime}$. Let $w \in \mathscr{V}$. Then, since $w$ is Bochner measurable, there is a sequence of simple functions $\left\{w_{n}\right\}$ such that $w_{n}(t) \rightarrow w(t)$ a.e. $t \in \mathrm{I}$. Consequently, for each $n \in \mathbb{N}, t \mapsto A\left(w_{n}(t)\right)$ is a simple $V^{\prime}$-valued function and $A\left(w_{n}(t)\right) \rightarrow A(w(t))$ a.e. $t \in \mathrm{I}$; that is, $t \mapsto A(w(t))$ is weakly measurable, and since $V^{\prime}$ is separable $t \mapsto A(w(t))$ is measurable (see Corollary 1.1.2. in [2]). Also, $\int_{\mathrm{I}}|A(w(t))|_{V^{\prime}}^{p /(p-1)} \mathrm{d} t \leq C \int_{\mathrm{I}}|w(t)|_{V}^{p} \mathrm{~d} t$, and hence $\mathcal{A}(w) \in L^{p^{\prime}}\left(\mathrm{I} ; V^{\prime}\right)$, with $\frac{1}{p}+\frac{1}{p^{\prime}}=1$, for each $w \in L^{p}(\mathrm{I} ; V)$ and H3 is satisfied. Further, for $u, v \in \mathscr{V}$, we have $(A(u(t))-A(v(t)), u(t)-v(t))_{V} \geq c|u(t)-v(t)|_{V}^{p}$ for a.e. $t \in \mathrm{I}$, and integration gives $(\mathcal{A}(u)-\mathcal{A}(v), u-v)_{\mathscr{V}} \geq c|u-v|_{\mathscr{V}}^{p}$, i.e., $\mathcal{A}$ satisfies H1. Let $u, v, w \in \mathscr{V}$ and $\eta \in[0,1]$; then $\left|(A(u(t)+\eta v(t)), w(t))_{V}\right| \leq C\left(|u(t)|_{V}+\eta|v(t)|_{V}\right)^{p-1}|w(t)|_{V}$, and hence, by Young's inequality,

$$
\begin{aligned}
\left|(A(u(t)+\eta v(t)), w(t))_{V}\right| & \leq C\left(\frac{\left(|u(t)|_{V}+\eta|v(t)|_{V}\right)^{p}}{p^{\prime}}+\frac{|w(t)|_{V}^{p}}{p}\right) \\
& \leq C\left(\frac{\left(|u(t)|_{V}+|v(t)|_{V}\right)^{p}}{p^{\prime}}+\frac{|w(t)|_{V}^{p}}{p}\right) .
\end{aligned}
$$

Finally, since the right-hand side of the above inequality belongs to $L^{1}(\mathrm{I} ; \mathbb{R})$ and if $\eta_{n} \rightarrow \eta$, then $\left(A\left(u(t)+\eta_{n} v(t)\right), w(t)\right)_{V} \rightarrow(A(u(t)+\eta v(t)), w(t))_{V}$ as $n \rightarrow \infty$ a.e. $t \in \mathrm{I}$ by the hemicontinuity of $A: V \rightarrow V^{\prime}$. The hemicontinuity of $\mathcal{A}$ (H2) follows from an application of the analogue of Lebesgue's bounded convergence theorem for the Bochner integral.

A typical example for the previous proposition is the following.

Example 2.2. Let $V=W_{0}^{1, p}(\Omega)$ with $p \geq 2, \Omega$ some bounded domain, and let $A=-\Delta_{p}: V \rightarrow V^{\prime}$, the negative $p$-Laplacian, i.e., $-\Delta_{p}$ is defined as

$$
\left(-\Delta_{p}(u), v\right)_{V}=\int_{\Omega}|\nabla u(\mathbf{x})|^{p-2}(\nabla u(\mathbf{x}) \cdot \nabla v(\mathbf{x})) \mathrm{d} \mathbf{x} \quad \forall u, v \in W_{0}^{1, p}(\Omega) .
$$

The map $A=-\Delta_{p}$ satisfies all hypotheses of the previous proposition. In fact, hypothesis H1 follows from the vector inequality $\left\langle|\mathbf{x}|^{p-2} \mathbf{x}-|\mathbf{y}|^{p-2} \mathbf{y}, \mathbf{x}-\mathbf{y}\right\rangle_{\mathbb{R}^{N}} \geq c \mid \mathbf{x}-$ $\left.\mathbf{y}\right|_{\mathbb{R}^{N}} ^{p}$ for some $c>0$ and for all $\mathbf{x}, \mathbf{y} \in \mathbb{R}^{N}$. The map $u \mapsto-\Delta_{p}(u)$ is Fréchet differentiable (as a mapping from $W_{0}^{1, p}(\Omega)$ with an image in $W^{-1, p^{\prime}}(\Omega)$; see [19]) and hence H2 holds true. Further, we have $\left|\left(-\Delta_{p}(u), v\right)_{W_{0}^{1, p}(\Omega)}\right| \leq \int_{\Omega}|\nabla u(\mathbf{x})|^{p-1}|\nabla v(\mathbf{x})| \mathrm{d} \mathbf{x} \leq$ $|u|_{W_{0}^{1, p}(\Omega)}^{p-1}|v|_{W_{0}^{1, p}(\Omega)}$, which proves the stronger version of H3 used in the previous proposition. Therefore, the time realization $(\mathcal{A}(v))(t)=-\Delta_{p}(v(t))$ for $v \in L^{p}\left(\mathrm{I} ; W_{0}^{1, p}\right.$ $(\Omega))$, i.e.,

$$
(\mathcal{A}(u), v)_{\mathscr{V}}=\int_{\mathrm{I}}\left(\int_{\Omega}|(\nabla u(t))(\mathbf{x})|^{p-2}((\nabla u(t))(\mathbf{x}) \cdot(\nabla v(t))(\mathbf{x})) \mathrm{d} \mathbf{x}\right) \mathrm{d} t
$$

for all $u, v \in L^{p}\left(\mathrm{I} ; W_{0}^{1, p}(\Omega)\right)$, satisfies $\mathrm{H} 1, \mathrm{H} 2$, and $\mathrm{H} 3$. 
In order to introduce some form of "time derivative," we make use of $C_{0}$-semigroup theory. More specifically, we consider a general framework which includes the "time derivative" as one possible operator choice. For that purpose, we assume in the following that $-L$ is the infinitesimal generator of a $C_{0}$-semigroup $S(\tau)$ in $\mathscr{V}, \mathscr{H}, \mathscr{V}^{\prime}$ (of contractions in $\mathscr{H}$, i.e., $|S(\tau)|_{\mathscr{H}} \leq 1$ for $\tau \geq 0$ ) with domains $\mathscr{D}(L ; \mathscr{V}), \mathscr{D}(L ; \mathscr{H})$ and $\mathscr{D}\left(L ; \mathscr{V}^{\prime}\right)$, respectively; see [31] for the concept of a $C_{0}$-semigroup. We have,

$$
\mathscr{D}(L ; \mathscr{V}):=\left\{v \in \mathscr{V}: \lim _{\tau \downarrow 0} \frac{S(\tau) v-v}{\tau} \text { exists in } \mathscr{V}\right\}
$$

where $\mathscr{D}(L ; \mathscr{H})$ and $\mathscr{D}\left(L ; \mathscr{V}^{\prime}\right)$ are defined similarly.

Example 2.3. Let $\mathscr{V}=L^{p}(\mathrm{I} ; X)$, for $\mathrm{I}=(0, T)$ with $0<T \leq \infty$, with $X$ a Banach space. For $f \in \mathscr{V}$, let $S(\tau)$ be defined by

$$
(S(\tau) f)(t)= \begin{cases}f(t-\tau), & \tau<t<T \\ 0 & \text { otherwise }\end{cases}
$$

Clearly $S(0)=I=\mathrm{id}, S\left(\tau_{1}+\tau_{2}\right)=S\left(\tau_{1}\right) S\left(\tau_{2}\right)$, and $\lim _{\tau \downarrow 0} S(\tau) f=f$ in $\mathscr{V}$. Hence $S(\tau)$ is a $C_{0}$-semigroup over $\mathscr{V}$. Moreover, $S(\tau)$ is a $C_{0}$-semigroup of contractions since $|S(\tau) f|_{\mathscr{V}} \leq|f|_{\mathscr{V}}$, which is not uniformly continuous.

Suppose that $\mathscr{C}$ is a closed and convex subset of $\mathscr{V}$ and $0 \in \mathscr{C}$. Consider a mapping $\mathscr{K}: \mathscr{C} \rightarrow 2^{\mathscr{V}}$ such that for each $v \in \mathscr{C}, \mathscr{K}(v)$ is a closed and convex subset of $\mathscr{V}$ and $0 \in \mathscr{K}(v)$. Let $f \in \mathscr{V}^{\prime}$ and $\mathcal{A}: \mathscr{V} \rightarrow \mathscr{V}^{\prime}$; then we define the problem (P $\left.\mathrm{P}_{\mathrm{QVI}}\right)$ as the following parabolic QVI.

\section{Problem $\left(\mathrm{P}_{\mathrm{QVI}}\right)$.}

$\left(\mathrm{P}_{\mathrm{QVI}}\right) \quad$ Find $u \in \mathscr{K}(u) \cap \mathscr{D}\left(L ; \mathscr{V}^{\prime}\right):(L u+\mathcal{A}(u)-f, v-u)_{\mathscr{V}} \geq 0 \quad \forall v \in \mathscr{K}(u)$.

The space $\mathscr{V}$ usually is considered to be a Banach space of mappings of the type $f: \mathrm{I} \rightarrow V$, where $\mathrm{I}=(0, T)$ with $0<T \leq \infty$ and $V$ is a separable reflexive Banach space. Then a general form of $\mathscr{K}(\cdot)$ is given by

$$
\mathscr{K}(v)=\{w \in \mathscr{V}: w(t) \in \mathbf{K}(v, t) \text { a.e. } t \in \mathrm{I}\},
$$

where $\mathbf{K}: \mathscr{V} \times \mathrm{I} \rightarrow 2^{V}$ and, for each $w \in \mathscr{V}$ and $t \in \mathrm{I}, \mathbf{K}(w, t)$ is a closed, convex subset of $V$ with $0 \in \mathbf{K}(w, t)$.

The most common form of $\mathbf{K}$ is the following. Let $G \in \mathscr{L}(V, W)$, where $W$ is a Banach space of functions over some domain $\Omega \subset \mathbb{R}^{N}$ and with range in $\mathbb{R}^{l}, l \in \mathbb{N}$. Let $\Phi: \mathscr{V} \times \mathrm{I} \rightarrow L_{\nu}^{\infty}(\Omega)$ so that $(\Phi(v, t))(\mathbf{x}) \geq \nu>0$ a.e. $t \in \mathrm{I}, \mathbf{x} \in \Omega$, and

$$
\mathbf{K}(v, t):=\left\{y \in V:|(G y)(\mathbf{x})|_{\mathbb{R}^{l}} \leq(\Phi(v, t))(\mathbf{x}) \text { a.e. in } \Omega\right\} .
$$

Example 2.4. Let $\mathscr{V}=L^{2}(\mathrm{I} ; V)$ and $\mathscr{H}=L^{2}(\mathrm{I} ; H)$, where $\left(V, H, V^{\prime}\right) \equiv\left(H_{0}^{1}(\Omega)\right.$, $\left.L^{2}(\Omega), H^{-1}(\Omega)\right)$ is a Gelfand triple. Consider $G \equiv \nabla \in \mathscr{L}\left(H_{0}^{1}(\Omega), L^{2}(\Omega)^{N}\right)$, and let $\Phi(v, t) \equiv \varphi \in L_{\nu}^{\infty}(\Omega)$ (i.e., $\Phi(v, t)$ is constant over $\mathscr{V} \times \mathrm{I}$ ). Hence, we have $\mathbf{K}(v, t) \equiv \mathbf{K}$ and

$$
\mathbf{K}:=\left\{y \in H_{0}^{1}(\Omega):|(\nabla y)(\mathbf{x})|_{\mathbb{R}^{N}} \leq \varphi(\mathbf{x}) \text { a.e. in } \Omega\right\} .
$$

Copyright $@$ by SIAM. Unauthorized reproduction of this article is prohibited. 
3. Theoretical framework. For practical realizations and the numerical solution of $\left(\mathrm{P}_{\mathrm{QVI}}\right)$, we are interested in approximating elements in the spaces of the Gelfand triple $\left(\mathscr{V}, \mathscr{H}, \mathscr{V}^{\prime}\right)$. For this purpose, we consider approximating spaces. Indeed, suppose that for each $n \in \mathbb{N}, \mathscr{V}_{n}$ is a reflexive separable Banach space and $\mathscr{H}_{n}$ is a separable Hilbert space for which $\left(\mathscr{V}_{n}, \mathscr{H}_{n}, \mathscr{V}_{n}^{\prime}\right)$ is also a Gelfand triple (where we also identify $\mathscr{H}_{n}$ with its dual $\mathscr{H}_{n}^{\prime}$ ). We invoke the following assumption that relates $\left(\mathscr{V}_{n}, \mathscr{H}_{n}, \mathscr{V}_{n}^{\prime}\right)$ to $\left(\mathscr{V}, \mathscr{H}, \mathscr{V}^{\prime}\right)$. that

Assumption 1. For $n \in \mathbb{N}$, there are $P_{n} \in \mathscr{L}\left(\mathscr{V}, \mathscr{V}_{n}\right)$ and $E_{n} \in \mathscr{L}\left(\mathscr{V}_{n}, \mathscr{V}\right)$ such

A1. $\exists M_{1}, M_{2}>0$ with $\left|P_{n}\right|_{\mathscr{L}\left(\mathscr{V}, \mathscr{V}_{n}\right)} \leq M_{1}$ and $\left|E_{n}\right|_{\mathscr{L}\left(\mathscr{V}_{n}, \mathscr{V}\right)} \leq M_{2}$ for all $n \in \mathbb{N}$;

A2. $\left|E_{n} P_{n} v-v\right|_{\mathscr{V}} \rightarrow 0$ as $n \rightarrow \infty$ for all $v \in \mathscr{V}$;

A3. $P_{n} E_{n}$ is the identity operator in $\mathscr{V}_{n}$.

The operators $P_{n}$ and $E_{n}$ are usually referred to as the "projection" and "extension" operators, respectively, and they define the relation between $\mathscr{V}$ and $\mathscr{V}_{n}$. Assumptions A1-A3 are common in analytical forms of the Trotter-Kato approximation theorem (see, for example, section 3.6 in [31] and Chapter 4 in [20]). We shall see at the end of this section that these operators are useful when approximating solutions to $\left(\mathrm{P}_{\mathrm{QVI}}\right)$. If there are projection and extension operators for $V$ and $V_{n}$ (some Banach spaces), then the same structure permeates through to the "time dependent" spaces $\mathscr{V}=L^{p}(\mathrm{I}, V)$ and $\mathscr{V}_{n}=L^{p}\left(\mathrm{I} ; V_{n}\right)$ as we observe in the following result.

Proposition 3.1. Let $V$ and $V_{n}$, with $n \in \mathbb{N}$, be Banach spaces. Suppose that $\pi_{n} \in \mathscr{L}\left(V, V_{n}\right)$ and $\varepsilon_{n} \in \mathscr{L}\left(V_{n}, V\right)$ are the corresponding projection and extension operators (fulfilling hypotheses A1-A3). Suppose also that $\mathscr{V}=L^{p}(\mathrm{I} ; V)$, with $1 \leq$ $p<\infty$ and $\mathrm{I}=(0, T)$ for $0<T \leq \infty$, and $\mathscr{V}_{n}=L^{p}\left(\mathrm{I} ; V_{n}\right)$ (for each $n \in \mathbb{N}$ ). Then, $\left(P_{n}(v)\right)(t)=\left(\pi_{n} v(t)\right)$ and $\left(E_{n}(w)\right)(t)=\left(\varepsilon_{n} w(t)\right)$ for $v \in \mathscr{V}$ and $w \in \mathscr{V}_{n}$ are the projection and extension operators associated with $\mathscr{V}$ and $\mathscr{V}_{n}$.

Proof. We first prove that if $v \in L^{p}(I ; V)$, then $P_{n} v \in L^{p}\left(I ; V_{n}\right)$. If $v: \mathrm{I} \rightarrow V$ is Bochner measurable, then there exists a sequence $\left\{v_{k}\right\}$ of simple functions $v_{k}: \mathrm{I} \rightarrow V$ such that $v_{k}(t) \rightarrow v(t)$ a.e. $t \in \mathrm{I}$ as $k \rightarrow \infty$. Clearly $t \mapsto\left(\pi_{n} v_{k}(t)\right)$ is a $V_{n}$-valued simple function and $\lim _{k \rightarrow \infty}\left|\pi_{n} v_{k}(t)-\pi_{n} v(t)\right|_{V_{n}} \leq M_{1} \lim _{k \rightarrow \infty}\left|v_{k}(t)-v(t)\right|_{V}=0$ a.e. $t \in \mathrm{I}$ (because $\left|\pi_{n}\right|_{\mathscr{L}\left(V, V_{n}\right)} \leq M_{1}$ by hypothesis A1). This implies the measurability of $t \mapsto \pi_{n} v(t)$, and since $\left|\pi_{n} v(t)\right|_{V_{n}}^{p} \leq M_{1}^{p}|v(t)|_{V}^{p}$, we have $\left(P_{n}(v)\right)(t)=\left(\pi_{n} v(t)\right) \in$ $L^{p}\left(\mathrm{I} ; V_{n}\right)$ for each $v \in L^{p}(\mathrm{I} ; V)$ and furthermore $\left|P_{n}\right|_{\mathscr{L}\left(\mathscr{V}, \mathscr{V}_{n}\right)} \leq M_{1}$. A similar argument proves the analogous result for $\left(E_{n}(v)\right)(t)=\left(\varepsilon_{n} w(t)\right)$ and $\left|E_{n}\right|_{\mathscr{L}\left(\mathscr{V}, \mathscr{V}_{n}\right)} \leq M_{2}$ (where $|\varepsilon|_{\mathscr{L}\left(V_{n}, V\right)} \leq M_{2}$ ), i.e., $P_{n}$ and $E_{n}$ satisfy A1.

If $v \in L^{p}(\mathrm{I} ; V)$, then $\left|\varepsilon_{n} \pi_{n} v(t)-v(t)\right|_{V}^{p} \rightarrow 0$ a.e. $t \in \mathrm{I}$ as $n \rightarrow \infty$ and $\mid \varepsilon_{n} \pi_{n} v(t)-$ $\left.v(t)\right|_{V} ^{p} \leq\left(M_{1} M_{2}+1\right)^{p}|v(t)|_{V}^{p} \in L^{1}(\mathrm{I} ; \mathbb{R})$, and by Lebesgue's bounded convergence theorem, we have A2. Finally, A3 follows readily.

We show that a solution to $\left(\mathrm{P}_{\mathrm{QVI}}\right)$ in $\mathscr{V}$ may be approximated by similar, but easier-to-solve, QVI-problems in $\mathscr{V}_{n}$. For this purpose we invoke hypotheses on $\mathcal{A}_{n}$ and $L_{n}$, the operators that play the analogous roles of $\mathcal{A}$ and $L$, but on $\mathscr{V}_{n}$. We first establish structural and stability properties of the sequences $\left\{\mathcal{A}_{n}\right\}$ and $\left\{L_{n}\right\}$.

We assume that we are given a sequence of mappings $\left\{\mathcal{A}_{n}\right\}$ such that for each $n \in \mathbb{N}$ we have that $\mathcal{A}_{n}: \mathscr{V}_{n} \rightarrow \mathscr{V}_{n}^{\prime}$ satisfies hypotheses H2 and H3. Moreover, we assume the following:

H1-n. There are sequences $\left\{c_{n}\right\}$ and $\left\{r_{n}\right\}$ such that $c_{n} \geq \bar{c}>0, r_{n} \geq \bar{r}>1$, and

$$
\left(\mathcal{A}_{n}(u)-\mathcal{A}_{n}(v), u-v\right)_{\mathscr{V}_{n}} \geq c_{n}|u-v|_{\mathscr{V}_{n}}^{r_{n}} \quad \forall u, v \in \mathscr{V}_{n} \text { and all } n \in \mathbb{N} .
$$

A sequence $\left\{L_{n}\right\} \subset \mathscr{L}\left(\mathscr{V}_{n}^{\prime}, \mathscr{V}_{n}^{\prime}\right)$ is used to approximate $L$. For each $L_{n}$ we allow a rather general form as specified next. 
H4-n. There exists $\left\{\rho_{n}\right\} \subset \mathbb{R}^{+}$such that $\lim _{n \rightarrow \infty} \rho_{n}=0$, and $\left\{F\left(\rho_{n}\right)\right\}$, a sequence of operators with $F\left(\rho_{n}\right) \in \mathscr{L}\left(\mathscr{V}_{n}^{\prime}, \mathscr{V}_{n}^{\prime}\right), F\left(\rho_{n}\right) \in \mathscr{L}\left(\mathscr{H}_{n}, \mathscr{H}_{n}\right)$, and $\left|F\left(\rho_{n}\right)\right|_{\mathscr{L}\left(\mathscr{H}_{n}\right)} \leq 1$ for all $n \in \mathbb{N}$. Suppose $L_{n}: \mathscr{V}_{n}^{\prime} \rightarrow \mathscr{V}_{n}^{\prime}$ is defined as

$$
L_{n}:=\frac{I-F\left(\rho_{n}\right)}{\rho_{n}} .
$$

Hypotheses H1-n and H4-n allow a wide range of possible sequences $\left\{\mathcal{A}_{n}\right\}$ and $\left\{L_{n}\right\}$. The simplest application is given in the following example.

Example 3.2. Let $S(\tau)$ be a $C_{0}$-semigroup over each of the spaces of the Gelfand triple $\left(\mathscr{V}, \mathscr{H}, \mathscr{V}^{\prime}\right)$ and of contractions over $\mathscr{H}$. Suppose also that $\mathcal{A}: \mathscr{V} \rightarrow \mathscr{V}^{\prime}$ satisfies H1, H2, and H3. Let $\left\{\rho_{n}\right\} \subset \mathbb{R}^{+}$such that $\lim _{n \rightarrow \infty} \rho_{n}=0$, and let $\mathscr{V}_{n}=\mathscr{V}$ and $\mathscr{H}_{n}=\mathscr{H}$ for each $n \in \mathbb{N}$. Then $L_{n}$ can be chosen as $L_{n}=\frac{I-S\left(\rho_{n}\right)}{\rho_{n}}$ and $\mathcal{A}_{n}=\mathcal{A}$. Furthermore, in this case if $v \in \mathscr{D}\left(L ; \mathscr{V}^{\prime}\right) \cap \mathscr{V}$, then $L_{n} v \rightarrow L v$ and $\mathcal{A}_{n} v \rightarrow \mathcal{A} v$, both in $\mathscr{V}^{\prime}$, as $n \rightarrow \infty$.

The situation considered in Example 3.2 is generalized by the following definition. In fact, we now define the type of convergence required for $L_{n}, \mathcal{A}_{n}$, and $f_{n}$ that is used below in the proof of existence and approximation of QVIs.

DeFinition 3.3 ( $\mathbf{\Lambda}$-convergence). Let $-L$ be a linear operator on $\mathscr{V}^{\prime}$ with domain $\mathscr{D}\left(L ; \mathscr{V}^{\prime}\right), f \in \mathscr{V}^{\prime}$, and $\mathcal{A}: \mathscr{V} \rightarrow \mathscr{V}^{\prime}$. For each $n \in \mathbb{N}$, let $-L_{n} \in \mathscr{L}\left(\mathscr{V}_{n}^{\prime}, \mathscr{V}_{n}^{\prime}\right), f_{n} \in \mathscr{V}_{n}^{\prime}$, and $\mathcal{A}_{n}: \mathscr{V}_{n} \rightarrow \mathscr{V}_{n}^{\prime}$. Also, let $P_{n}^{\prime} \in \mathscr{L}\left(\mathscr{V}_{n}^{\prime}, \mathscr{V}^{\prime}\right)$ denote the transpose (or conjugate) operator of $P_{n} \in \mathscr{L}\left(\mathscr{V}, \mathscr{V}_{n}\right)$. We say that the sequence of triples $\left\{\left(L_{n}, f_{n}, \mathcal{A}_{n}\right)\right\} \mathbf{\Delta}$ converges to $(L, f, \mathcal{A})$ and denote this by $\left(L_{n}, \mathcal{A}_{n}, f_{n}\right) \stackrel{\mathbf{\Delta}}{\longrightarrow}(L, \mathcal{A}, f)$ if

i. $\lim _{n \rightarrow \infty} P_{n}^{\prime} L_{n} P_{n} v=L v$ in $\mathscr{V}^{\prime} \forall v \in \mathscr{D}\left(L ; \mathscr{V}^{\prime}\right) \cap \mathscr{V}$;

ii. $\lim _{n \rightarrow \infty} P_{n}^{\prime} f_{n}=f$, in $\mathscr{V}^{\prime}$;

iii. the map $\mathcal{A}_{n}: \mathscr{V} \rightarrow \mathscr{V}^{\prime}$ defined as $\tilde{\mathcal{A}}_{n}(w):=P_{n}^{\prime} \mathcal{A}_{n}\left(P_{n} w\right)$ for $w \in \mathscr{V}$ satisfies

$$
\lim _{n \rightarrow \infty} \tilde{\mathcal{A}}_{n}(v)=\mathcal{A}(v) \quad \text { in } \mathscr{V}^{\prime}
$$

uniformly on bounded sets for $v \in \mathscr{V}$.

Condition $\mathrm{i}$ in the above definition is used in the approximation theory of $C_{0^{-}}$ semigroups (see section 3.6 in [31] and Chapter 4 in [20]) and determines that the realization of $-L$ (on part of its domain) can be approximated using $-L_{n}$. Condition ii in Definition 3.3 is a form of strong convergence of the forcing terms, and iii in Definition 3.3 is a form of uniform convergence of the maps $\mathcal{A}_{n}$ to $\mathcal{A}$ as can be observed in the following lemma.

Lemma 1. Let $\left\{\left(L_{n}, f_{n}, \mathcal{A}_{n}\right)\right\}$ be defined as in Definition 3.3, and assume that $\left(L_{n}, \mathcal{A}_{n}, f_{n}\right)$

$\stackrel{\Delta}{\longrightarrow}(L, \mathcal{A}, f)$ and that Assumption 1 holds. Then, we have the following:

(1) The sequence $\left\{\left|f_{n}\right|_{\mathscr{V}_{n}^{\prime}}\right\}$ is uniformly bounded.

(2) If $\mathcal{A}$ satisfies $\mathrm{H} 3, v_{n} \rightarrow v$, and $w_{n} \rightarrow w$ in $\mathscr{V}$, respectively, then

$$
\varlimsup_{n \rightarrow \infty}\left(\mathcal{A}\left(v_{n}\right), v_{n}-w\right)_{\mathscr{V}}=\varlimsup_{n \rightarrow \infty}\left(\tilde{\mathcal{A}}_{n}\left(v_{n}\right), v_{n}-w_{n}\right)_{\mathscr{V}}
$$

and $\underline{\lim }_{n \rightarrow \infty}\left(\mathcal{A}\left(v_{n}\right), v_{n}-w\right)_{\mathscr{V}}=\underline{\lim }_{n \rightarrow \infty}\left(\tilde{\mathcal{A}}_{n}\left(v_{n}\right), v_{n}-w_{n}\right)_{\mathscr{V}}$.

Proof. Since $P_{n} E_{n}$ is the identity operator in $\mathscr{V}_{n}$ (A2 in Assumption 1), we observe that

$$
\left|f_{n}\right|_{\mathscr{V}_{n}^{\prime}}=\sup _{|z|_{\mathscr{V}_{n}}=1}\left|\left(f_{n}, z\right)_{\mathscr{V}_{n}}\right|=\sup _{|z|_{\mathscr{V}_{n}}=1}\left|\left(P_{n}^{\prime} f_{n}, E_{n} z\right)_{\mathscr{V}}\right|
$$

Copyright (c) by SIAM. Unauthorized reproduction of this article is prohibited. 
Also, $\left|\left(P_{n}^{\prime} f_{n}, E_{n} z\right)_{\mathscr{V}}\right| \leq\left|P_{n}^{\prime} f_{n}\right|_{\mathscr{V}^{\prime}}\left|E_{n}\right|_{\mathscr{L}\left(\mathscr{V}_{n}, \mathscr{V}\right)}|z|_{\mathscr{V}_{n}}$, and then $\left|f_{n}\right|_{\mathscr{V}_{n}^{\prime}} \leq M_{2}\left|P_{n}^{\prime} f_{n}\right|_{\mathscr{V}^{\prime}}$ (because $\left|E_{n}\right|_{\mathscr{L}\left(\mathscr{V}_{n}, \mathscr{V}\right)} \leq M_{2}$ for all $n \in \mathbb{N}$ due to A1). Finally, $P_{n}^{\prime} f_{n} \rightarrow f$ in $\mathscr{V}^{\prime}$ implies that $\left\{\left|P_{n}^{\prime} f_{n}\right|_{\mathscr{V}^{\prime}}\right\}$ is uniformly bounded and the same holds true for $\left\{\left|f_{n}\right|_{\mathscr{Y}_{n}^{\prime}}\right\}$. This proves (1).

We clearly have

$$
\begin{aligned}
& \left(\mathcal{A}\left(v_{n}\right), v_{n}-w\right)_{\mathscr{V}} \\
& \quad=\left(\tilde{\mathcal{A}}_{n}\left(v_{n}\right), v_{n}-w_{n}\right)_{\mathscr{V}}+\left(\tilde{\mathcal{A}}_{n}\left(v_{n}\right), w_{n}-w\right)_{\mathscr{V}}+\left(\mathcal{A}\left(v_{n}\right)-\tilde{\mathcal{A}}_{n}\left(v_{n}\right), v_{n}-w\right)_{\mathscr{V}} .
\end{aligned}
$$

Since $v_{n} \rightarrow v$ in $\mathscr{V}$, the sequence $\left\{v_{n}\right\}$ is uniformly bounded and, therefore, we have $\lim _{n \rightarrow \infty}\left|\tilde{\mathcal{A}}_{n}\left(v_{n}\right)-\mathcal{A}\left(v_{n}\right)\right|_{\mathscr{V}^{\prime}}=0$ (by iii in Definition 3.3). Also, $\left\{\mathcal{A}\left(v_{n}\right)\right\}$ is bounded in $\mathscr{V}^{\prime}$ (because of H3) and hence $\left|\tilde{\mathcal{A}}_{n}\left(v_{n}\right)\right|_{\mathscr{V}^{\prime}} \leq C$ for some $C>0$ and all $n \in \mathbb{N}$. Then, from (3.2), we have

$$
\left(\mathcal{A}\left(v_{n}\right), v_{n}-w\right)_{\mathscr{V}} \leq\left(\tilde{\mathcal{A}}_{n}\left(v_{n}\right), v_{n}-w_{n}\right)_{\mathscr{V}}+C\left|w_{n}-w\right|_{\mathscr{V}}+o(1)\left|v_{n}-w\right|_{\mathscr{V}}
$$

and

$$
\left(\tilde{\mathcal{A}}_{n}\left(v_{n}\right), v_{n}-w_{n}\right)_{\mathscr{V}} \leq\left(\mathcal{A}\left(v_{n}\right), v_{n}-w\right)_{\mathscr{V}}+C\left|w_{n}-w\right|_{\mathscr{V}}+o(1)\left|v_{n}-w\right|_{\mathscr{V}}
$$

Since $w_{n} \rightarrow w$ in $\mathscr{V}$ and $\left|v_{n}-w\right|_{\mathscr{V}}$ is uniformly bounded, we have that

$$
\varlimsup_{n \rightarrow \infty}\left(\mathcal{A}\left(v_{n}\right), v_{n}-w\right)_{\mathscr{V}} \leq \varlimsup_{n \rightarrow \infty}\left(\tilde{\mathcal{A}}_{n}\left(v_{n}\right), v_{n}-w_{n}\right)_{\mathscr{V}}
$$

and

$$
\varlimsup_{n \rightarrow \infty}\left(\tilde{\mathcal{A}}_{n}\left(v_{n}\right), v_{n}-w_{n}\right)_{\mathscr{V}} \leq \varlimsup_{n \rightarrow \infty}\left(\mathcal{A}\left(v_{n}\right), v_{n}-w\right)_{\mathscr{V}}
$$

from (3.3) and (3.4), respectively. The same holds when "im" is exchanged for "lim." This proves (2).

3.1. Conditions on $\mathscr{K}_{n}(\cdot), \mathscr{K}(\cdot)$, and $S(\tau)$. As we have already defined the type of convergence needed for $\left\{L_{n}\right\},\left\{\mathcal{A}_{n}\right\}$, and $\left\{f_{n}\right\}$ in Definition 3.3, we next specify the notions required for the convergence of the sequences of set-valued mappings $\left\{\mathscr{K}_{n}(\cdot)\right\}$ towards $\mathscr{K}(\cdot)$. We divide the convergence assumptions into two different sets of conditions. Assumption 2 concerns solely $\left\{\mathscr{K}_{n}(\cdot)\right\}$ and $\mathscr{K}(\cdot)$ whereas Assumption 3 also considers certain compatibility conditions among $\left\{\mathscr{K}_{n}(\cdot)\right\}, \mathscr{K}(\cdot)$, and the $C_{0^{-}}$ semigroup $S(\tau)$ generated by $-L$.

Throughout this section, we assume that $\mathscr{K}: \mathscr{C} \subset \mathscr{V} \rightarrow 2^{\mathscr{V}}$, where $\mathscr{C}$ is a closed, convex subset of $\mathscr{V}$ with $0 \in \mathscr{C}$. Also for each $v \in \mathscr{C}, \mathscr{K}(v)$ is a closed, convex subset of $\mathscr{V}$ with $0 \in \mathscr{K}(v)$. Similarly, for the sequence $\left\{\mathscr{K}_{n}(\cdot)\right\}$, we suppose that for each $n \in \mathbb{N}, \mathscr{K}_{n}: \mathscr{C}_{n} \subset \mathscr{V}_{n} \rightarrow 2^{\mathscr{V}_{n}}$, where $\mathscr{C}_{n}$ is a closed, convex subset of $\mathscr{V}_{n}$ such that $0 \in \mathscr{C}_{n}$, and for each $v \in \mathscr{C}_{n}, \mathscr{K}_{n}(v)$ is also a closed, convex subset of $\mathscr{V}_{n}$ with $0 \in \mathscr{K}_{n}(v)$. For the sake of simplicity, we suppose that the following assumption holds.

H5. There exists a sequence $\left\{R_{n}\right\} \subset \mathbb{R}^{+}$such that $\bar{B}_{R_{n}}\left(0 ; \mathscr{V}_{n}\right) \subset \mathscr{C}_{n}$ for all $n \in \mathbb{N}$. In addition, we invoke the following assumption.

Assumption 2.

A4. If the sequence $\left\{v_{k}\right\} \subset \mathscr{C}_{n}$ satisfies $v_{k} \rightarrow v$ in $\mathscr{V}_{n}$ as $k \rightarrow \infty$, then $\mathscr{K}_{n}\left(v_{k}\right) \rightarrow$ $\mathscr{K}_{n}(v)$ in the Mosco sense as $k \rightarrow \infty$ (see Definition 1.1).

A5. If $v_{n} \in \mathscr{K}_{n}\left(v_{n}\right)$ and $E_{n} v_{n} \rightarrow v$ in $\mathscr{V}$ for $n \in \hat{\mathbb{N}} \subset \mathbb{N}$, then $v \in \mathscr{K}(v)$. 
If we consider that $\mathscr{V}_{n}$ is a finite dimensional subset of $\mathscr{V}$, then assumption A4 is reduced to a much weaker form: $\mathscr{K}_{n}\left(v_{k}\right) \rightarrow \mathscr{K}_{n}(v)$ in the Mosco sense when $v_{k} \rightarrow v$ as $k \rightarrow \infty$ in $\mathscr{V}_{n}$. In the case when $\mathscr{V}_{n} \subset \mathscr{V}$, then assumption A5 is only one of the well-known conditions of the Mosco convergence of the sets $\mathscr{K}_{n}\left(v_{n}\right)$ towards $\mathscr{K}(v)$ (see Definition 1.1).

The following lemma will be used later; a proof may be found in section 9.2, Chapter 2 in [25]. Besides its analytical implications, it basically sets a bound on $\tau \mapsto \inf _{w \in \mathscr{K}}|S(\tau) v-w|$ uniformly for $v \in \mathscr{K}$.

LEMMA 2. Let $\mathscr{K}$ be a nonempty, closed, and convex subset of $\mathscr{V}$ and suppose

$$
\exists a>0 \text { such that } \quad e^{-a \tau} S(\tau) \mathscr{K} \subset \mathscr{K} \quad \forall \tau \geq 0 .
$$

Then the net $\left\{v_{\epsilon}\right\}$, with $\epsilon \in(0,1 / a)$, defined as $v_{\epsilon}=(1-a \epsilon)(I+\epsilon L)^{-1} v$ for $v \in \mathscr{K}$, satisfies $v_{\epsilon} \in \mathscr{D}\left(L ; \mathscr{V}^{\prime}\right) \cap \mathscr{K}$ and

(1) $\lim _{\epsilon \downarrow 0} v_{\epsilon}=v$ in $\mathscr{V}$

(2) $\left(L v_{\epsilon}, v_{\epsilon}-v\right)_{\mathscr{V}} \leq 0$ for $\epsilon \in(0,1 / a)$.

If $S(\tau)$ and $\mathscr{K}$ are such that for each $v \in \mathscr{K}$ there exists a net $\left\{v_{\epsilon}\right\}$ with $v_{\epsilon} \in$ $\mathscr{D}\left(L ; \mathscr{V}^{\prime}\right) \cap \mathscr{K}$ and (1) and (2) satisfied, then we call $S(\tau)$ and $\mathscr{K}$ compatible or say that they satisfy the compatibility conditions. Weaker conditions for this to hold are described in [7]. However, condition (3.5) is used in this work. We are now in place to determine the required assumptions on $\left\{\mathscr{K}_{n}(\cdot)\right\}, \mathscr{K}(\cdot)$, and $-L$ in order to obtain a useful approximating scheme.

Assumption 3.

A6. For each $v \in \mathscr{C}, \mathscr{K}(v)$ is compatible with the semigroup $S(\tau)$.

A7. If $\left\{v_{n}\right\} \subset \mathscr{V}_{n}$ such that $E_{n} v_{n} \rightarrow v$ in $\mathscr{V}$ for $n \in \hat{\mathbb{N}} \subset \mathbb{N}$ and $w \in \mathscr{D}\left(L ; \mathscr{V}^{\prime}\right) \cap$ $\mathscr{K}(v)$, then there is a sequence $\left\{\eta_{n}\right\} \subset \mathbb{R}^{+}$such that $\lim _{n \rightarrow \infty} \eta_{n}=1$ and $\eta_{n} P_{n} w \in$ $\mathscr{K}_{n}\left(v_{n}\right)$ for $n \in \hat{\mathbb{N}} \subset \mathbb{N}$.

Assumption A6 is a crucial part of the approximating scheme and it was first invoked (to the best of our knowledge) by Brézis [7] and Lions [25]. Assumption A7 is a form of a stability condition and it is simple to verify for a wide range of gradienttype constraint sets (in the elliptic case) as shown in [19]. We are now able to state the approximation theorem.

TheOREm 1. Suppose that $\mathcal{A}: \mathscr{V} \rightarrow \mathscr{V}^{\prime}$ satisfies $\mathrm{H} 1, \mathrm{H} 2$, and $\mathrm{H} 3$, that $\mathcal{A}_{n}: \mathscr{V}_{n} \rightarrow$ $\mathscr{V}_{n}^{\prime}$ satisfies $\mathrm{H} 1, \mathrm{H} 2$, and $\mathrm{H} 3-\mathrm{n}$, that $\left\{L_{n}\right\}$ satisfies $\mathrm{H} 4-\mathrm{n}$ and $\left\{f_{n}\right\} \subset \mathscr{V}^{\prime}$. Suppose also that assumptions 1, 2 and 3 hold (which is A1 through A7).

Then, if $f \in \mathscr{V}^{\prime}$ and $\left(L_{n}, \mathcal{A}_{n}, f_{n}\right) \stackrel{\boldsymbol{\iota}}{\longrightarrow}(L, \mathcal{A}, f)$ (Definition 3.3), and H5 holds for $R_{n}=\left(\left|f_{n}\right|_{\mathscr{Y}_{n}^{\prime}} / c_{n}\right)^{1 /\left(r_{n}-1\right)}$ (where $\left\{c_{n}\right\}$ and $\left\{r_{n}\right\}$ are the sequences of $\left.\mathrm{H} 3-\mathrm{n}\right)$, there then exists a solution $u \in \mathscr{V}$ to the problem

$$
\text { Find } u \in \mathscr{K}(u):(L v+\mathcal{A}(u)-f, v-u)_{\mathscr{V}} \geq 0 \quad \forall v \in \mathscr{K}(u) \cap \mathscr{D}\left(L ; \mathscr{V}^{\prime}\right) .
$$

Moreover, for each $n \in \mathbb{N}$, there exists an element $u_{n} \in \mathscr{V}_{n}$ that satisfies

$$
u_{n} \in \mathscr{K}_{n}\left(u_{n}\right):\left(L_{n} u_{n}+\mathcal{A}_{n}\left(u_{n}\right)-f_{n}, v-u_{n}\right) \mathscr{V}_{n} \geq 0 \quad \forall v \in \mathscr{K}_{n}\left(u_{n}\right),
$$

and the sequence $\left\{u_{n}\right\}$ fulfills $E_{n} u_{n} \rightarrow u$ in $\mathscr{V}$, along a subsequence.

Proof. Suppose $n \in \mathbb{N}$ is fixed. Let $\mathcal{B}_{n}:=L_{n}+\mathcal{A}_{n}$; then $\mathcal{B}_{n}: \mathscr{V}_{n} \rightarrow \mathscr{V}_{n}^{\prime}$ is uniformly monotone. In order to see this, let $v \in \mathscr{V}_{n}$. Then we have

$$
\left(L_{n} v, v\right)_{\mathscr{V}_{n}}=\left\langle L_{n} v, v\right\rangle_{\mathscr{H}_{n}}=\frac{1}{\rho_{n}}\left(|v|_{\mathscr{H}_{n}}^{2}-\left\langle F\left(\rho_{n}\right) v, v\right\rangle_{\mathscr{H}_{n}}\right) \geq 0
$$


because $\left|F\left(\rho_{n}\right)\right|_{\mathscr{L}\left(\mathscr{H}_{n}, \mathscr{H}_{n}\right)} \leq 1$ by hypothesis H4-n. The uniform monotonicity of $\mathcal{B}_{n}$ then follows from the Gelfand triple structure of $\left(\mathscr{V}_{n}, \mathscr{H}_{n}, \mathscr{V}_{n}^{\prime}\right)$ and the uniform monotonicity of $\mathcal{A}_{n}$ according to hypothesis H1-n. Since $\mathcal{A}_{n}$ is hemicontinuous and bounded by hypotheses $\mathrm{H} 1$ and $\mathrm{H} 2$, and $L_{n}=\left(I-F\left(\rho_{n}\right)\right) / \rho_{n}$ has this property as well since $F\left(\rho_{n}\right)$ is linear and bounded, we have that $\mathcal{B}_{n}$ is hemicontinuous and bounded. For each $w \in \mathscr{C}_{n} \subset \mathscr{V}_{n}$, the set $\mathscr{K}_{n}(w) \subset \mathscr{V}_{n}$ is closed, convex, and nonempty $(0 \in \mathscr{K}(w))$; hence, for each $f_{n} \in \mathscr{V}_{n}$, there exists a unique solution in $\mathscr{V}_{n}$ (see section 2.II in [37]) to the problem

$$
\text { Find } u \in \mathscr{K}_{n}(w):\left(\mathcal{B}_{n}(u)-f_{n}, v-u\right)_{\mathscr{V}_{n}} \geq 0 \quad \forall v \in \mathscr{K}_{n}(w) .
$$

We denote this solution by $S_{n}(w)$. The map $S_{n}: \mathscr{C}_{n} \subset \mathscr{V}_{n} \rightarrow \mathscr{V}_{n}$ is compact and continuous. Indeed, let the sequence $\left\{w_{k}\right\} \subset \mathscr{C}_{n}$ satisfy $w_{k} \rightarrow w$ in $\mathscr{V}_{n}$. Then by assumption A4, $\mathscr{K}_{n}\left(w_{k}\right) \rightarrow \mathscr{K}_{n}(w)$ in the sense of Mosco. Therefore, $S_{n}\left(w_{k}\right) \rightarrow$ $S_{n}(w)$ in $\mathscr{V}_{n}$ (see [29] or section 4:4 in [34]). Hence $S_{n}$ is completely continuous (and, therefore, also continuous) and since $\mathscr{V}_{n}$ is reflexive, $S_{n}$ is compact and continuous. Since $0 \in \mathscr{K}_{n}(w)$ and $\left(\mathcal{B}_{n}(v), v\right)_{\mathscr{V}_{n}} \geq\left(\mathcal{A}_{n}(v), v\right)_{\mathscr{V}_{n}} \geq c_{n}|v|_{\mathscr{V}_{n}}^{r_{n}}$ with $1<r_{n}, 0<c \leq c_{n}$ and $c_{n}, r_{n}$ uniformly bounded for $n \in \mathbb{N}$ (Hypothesis H1-n), then using $v=0$ in (3.8) yields $\left|S_{n}(w)\right|_{\mathscr{V}_{n}} \leq\left(\left|f_{n}\right|_{\mathscr{V}_{n}^{\prime}} / c_{n}\right)^{1 /\left(r_{n}-1\right)}=: R_{n}$. Since $\bar{B}_{R_{n}}\left(0 ; \mathscr{V}_{n}\right) \subset \mathscr{C}_{n}$, we have that $S_{n}\left(\mathscr{C}_{n}\right) \subset \mathscr{C}_{n}$. Hence, by Schauder's fixed-point theorem there exists $u_{n} \in \mathscr{C}_{n} \subset \mathscr{V}_{n}$ such that $u_{n}=S_{n}\left(u_{n}\right)$.

We now apply an argument similar to one usually used in the proof of Minty's lemma (see Lemma 1.5 in [22] or Lemma 4.2 in [34]). Define $\tilde{f}_{n}:=\mathcal{A}_{n}\left(u_{n}\right)-f_{n}$. Since $L_{n}$ is monotone, $0 \leq\left(L_{n} v-L_{n} u_{n}, v-u_{n}\right)_{\mathscr{V}_{n}}$ for all $v \in \mathscr{K}_{n}\left(u_{n}\right)$ and further

$0 \leq\left(L_{n} v+\tilde{f}_{n}-\left(L_{n} u_{n}+\tilde{f}_{n}\right), v-u_{n}\right)_{\mathscr{V}_{n}}=\left(L_{n} v+\tilde{f}_{n}, v-u_{n}\right)_{\mathscr{V}_{n}}-\left(L_{n} u_{n}+\tilde{f}_{n}, v-u_{n}\right)_{\mathscr{V}_{n}}$.

However, $\left(L_{n} u_{n}+\tilde{f}_{n}, v-u_{n}\right)_{\mathscr{V}_{n}}=\left(\mathcal{B}_{n}\left(u_{n}\right)-f_{n}, v-u\right)_{\mathscr{V}_{n}} \geq 0$ for all $v \in \mathscr{K}_{n}\left(u_{n}\right)$ (because of (3.8)) and hence we observe that $u_{n}=S_{n}\left(u_{n}\right)$ satisfies

$$
u_{n} \in \mathscr{K}_{n}\left(u_{n}\right):\left(L_{n} v+\mathcal{A}_{n}\left(u_{n}\right)-f_{n}, v-u_{n}\right) \mathscr{Y}_{n} \geq 0 \quad \forall v \in \mathscr{K}_{n}\left(u_{n}\right) .
$$

Since $\left(L_{n}, \mathcal{A}_{n}, f_{n}\right) \stackrel{\boldsymbol{\Delta}}{\longrightarrow}(L, \mathcal{A}, f)$ and $\left|u_{n}\right|_{\mathscr{V}_{n}} \leq\left(\left|f_{n}\right|_{\mathscr{V}_{n}^{\prime}} / c_{n}\right)^{1 /\left(r_{n}-1\right)},\left|u_{n}\right|_{\mathscr{V}_{n}}$ is uniformly bounded for $n \in \mathbb{N}$ (this follows from $c_{n} \geq \bar{c}>0, r_{n} \geq \bar{r}>1$ by Hypothesis H1-n and from the uniform boundedness of $\left\{\left|f_{n}\right|_{\mathscr{V}_{n}^{\prime}}\right\}$ by Lemma 1) and hence so is $\left|E_{n} u_{n}\right|_{\mathscr{V}}$ (because $\left|E_{n}\right|_{\mathscr{L}\left(\mathscr{V}_{n}, \mathscr{V}\right)} \leq M_{2}$ from A1). Similarly, recalling $\tilde{\mathcal{A}}_{n}(\cdot):=P_{n}^{\prime} \mathcal{A}_{n}\left(P_{n} \cdot\right): \mathscr{V} \rightarrow$ $\mathscr{V}^{\prime}$, we have that $\left|\tilde{\mathcal{A}}_{n}\left(E_{n} u_{n}\right)\right|_{\mathscr{V}^{\prime}}$ is also uniformly bounded in $n \in \mathbb{N}$ (as $\mathcal{A}\left(E_{n} u_{n}\right)$ is bounded due to $\mathrm{H} 3$ and $\lim _{n \rightarrow \infty}\left|\tilde{\mathcal{A}}_{n}\left(E_{n} v_{n}\right)-\mathcal{A}\left(E_{n} v_{n}\right)\right|_{\mathscr{V}^{\prime}}=0$ by iii in Definition 3.3). Because $\mathscr{V}$ is reflexive, it is inferred that

$$
E_{n} u_{n} \rightarrow u \text { in } \mathscr{V} \text { and } \tilde{\mathcal{A}}_{n}\left(E_{n} u_{n}\right) \rightarrow g \text { in } \mathscr{V}^{\prime}
$$

(for subsequences $\left\{E_{n_{k}} u_{n_{k}}\right\}$ and $\left\{\tilde{\mathcal{A}}_{n_{k}}\left(E_{n_{k}} u_{n_{k}}\right)\right\}$ that we denote, for the sake of brevity, as $\left\{E_{n} u_{n}\right\}$ and $\left.\left\{\tilde{\mathcal{A}}_{n}\left(E_{n} u_{n}\right)\right\}\right)$ for some $u \in \mathscr{V}$ and $g \in \mathscr{V}^{\prime}$.

Since $u_{n}=S_{n}\left(u_{n}\right)$ and $P_{n} E_{n}$ is the identity in $\mathscr{V}_{n}$ (Hypothesis A3), we have that

$$
\left(P_{n}^{\prime} L_{n} v+\tilde{\mathcal{A}}_{n}\left(E_{n} u_{n}\right)-P_{n}^{\prime} f_{n}, E_{n} v-E_{n} u_{n}\right)_{\mathscr{V}} \geq 0 \quad \forall v \in \mathscr{K}_{n}\left(u_{n}\right) .
$$

Let $w \in \mathscr{D}\left(L ; \mathscr{V}^{\prime}\right) \cap \mathscr{K}(u)$ be arbitrary. Since $E_{n} u_{n} \rightarrow u$ in $\mathscr{V}$, by A7 there exists a sequence $v_{n}=\eta_{n} P_{n} w \in \mathscr{K}_{n}\left(u_{n}\right)$ with $\lim _{n \rightarrow \infty} \eta_{n}=1$. Moreover, $E_{n} v_{n}=\eta_{n} E_{n} P_{n} w \rightarrow w$ 
as $n \rightarrow \infty$ in $\mathscr{V}$ (because of $\eta_{n} \rightarrow 1$ and $E_{n} P_{n} w \rightarrow w$ in $\mathscr{V}$ as $n \rightarrow \infty$; the latter follows from A2). Using $v=v_{n}$ in (3.9), we have

$$
\begin{aligned}
& \left(\tilde{\mathcal{A}}_{n}\left(E_{n} u_{n}\right), E_{n} u_{n}-u\right)_{\mathscr{V}} \\
& \quad \leq\left(\eta_{n} P_{n}^{\prime} L_{n} P_{n} w-P_{n}^{\prime} f_{n}, \eta_{n} E_{n} P_{n} w-E_{n} u_{n}\right)_{\mathscr{V}}+\left(\tilde{\mathcal{A}}_{n}\left(E_{n} u_{n}\right), \eta_{n} E_{n} P_{n} w-u\right)_{\mathscr{V}} .
\end{aligned}
$$

Therefore, since $\left(L_{n}, \mathcal{A}_{n}, f_{n}\right) \stackrel{\boldsymbol{\Delta}}{\longrightarrow}(L, \mathcal{A}, f), \eta_{n} E_{n} P_{n} w \rightarrow w$ and $E_{n} u_{n} \rightarrow u$ in $\mathscr{V}$. By Lemma 1, we have (from the previous inequality)

$$
\begin{aligned}
\varlimsup_{n \rightarrow \infty}\left(\mathcal{A}\left(E_{n} u_{n}\right), E_{n} u_{n}-u\right)_{\mathscr{V}} \\
=\varlimsup_{n \rightarrow \infty}\left(\tilde{\mathcal{A}}_{n}\left(E_{n} u_{n}\right), E_{n} u_{n}-u\right)_{\mathscr{V}} \leq(L w+g-f, w-u)_{\mathscr{V}} .
\end{aligned}
$$

Since $E_{n} u_{n} \rightarrow u$ and $u_{n} \in \mathscr{K}_{n}\left(u_{n}\right)$, then $u \in \mathscr{K}(u)$ (by A5). Next, choose $w=w_{\alpha}$ which belongs to $\mathscr{D}\left(L ; \mathscr{V}^{\prime}\right) \cap \mathscr{K}(u)$ with $\lim _{\alpha \rightarrow 0} w_{\alpha}=u$ and $\left(L w_{\alpha}, w_{\alpha}-u\right) \leq 0$ (which is possible due to the compatibility of $S(\tau)$ and $\mathscr{K}$ given in A6). Then, from the previous inequality, we obtain

$$
\varlimsup_{n \rightarrow \infty}\left(\mathcal{A}\left(E_{n} u_{n}\right), E_{n} u_{n}-u\right)_{\mathscr{V}} \leq 0 .
$$

Since $\mathcal{A}$ satisfies $\mathrm{H} 1$ and $\mathrm{H} 3$, it is also pseudomonotone (see the paragraph that follows the definitions of H1-H3 and see Proposition 2.3, Chapter II in [37] for a proof), and we have that

$$
(\mathcal{A}(u), u-v)_{\mathscr{V}} \leq \varliminf_{n \rightarrow \infty}\left(\mathcal{A}\left(E_{n} u_{n}\right), E_{n} u_{n}-v\right)_{\mathscr{V}} \quad \forall v \in \mathscr{V} .
$$

Again, let $w \in \mathscr{D}\left(L ; \mathscr{V}^{\prime}\right) \cap \mathscr{K}(u)$ be arbitrary; then there is a sequence $v_{n}=\eta_{n} P_{n} w \in$ $\mathscr{K}_{n}\left(u_{n}\right)$ and $E_{n} v_{n}=\eta_{n} E_{n} P_{n} w \rightarrow w$ as $n \rightarrow \infty$ in $\mathscr{V}$. Using $v=v_{n}$ in (3.9) and by applying Lemma 1 we get

$\varliminf_{n \rightarrow \infty}\left(\mathcal{A}\left(E_{n} u_{n}\right), E_{n} u_{n}-w\right)_{\mathscr{V}}=\varliminf_{n \rightarrow \infty}\left(\tilde{\mathcal{A}}_{n}\left(E_{n} u_{n}\right), E_{n} u_{n}-E_{n} v_{n}\right)_{\mathscr{V}} \leq(f-L w, u-w)_{\mathscr{V}}$.

Consequently, together with (3.12), this implies that $u \in \mathscr{V}$ satisfies

$$
u \in \mathscr{K}(u):(L w+\mathcal{A}(u)-f, w-u)_{\mathscr{V}} \geq 0 \quad \forall w \in \mathscr{D}\left(L ; \mathscr{V}^{\prime}\right) \cap \mathscr{K}(u) .
$$

Now, the uniform monotonicity of $\mathcal{A}$ (hypothesis H1) yields

$$
c\left|E_{n} u_{n}-u\right|_{\mathscr{V}}^{r} \leq\left(\mathcal{A}\left(E_{n} u_{n}\right)-\mathcal{A}(u), E_{n} u_{n}-u\right)_{\mathscr{V}} .
$$

Since $\left(\mathcal{A}(u), E_{n} u_{n}-u\right)_{\mathscr{V}} \rightarrow 0$ (because $E_{n} u_{n} \rightarrow u$ in $\left.\mathscr{V}\right)$, then $\overline{\lim }_{n \rightarrow \infty}\left(\mathcal{A}\left(E_{n} u_{n}\right)-\right.$ $\left.\mathcal{A}(u), E_{n} u_{n}-u\right)_{\mathscr{V}}=\varlimsup_{\lim _{n \rightarrow \infty}}\left(\mathcal{A}\left(E_{n} u_{n}\right), E_{n} u_{n}-u\right)_{\mathscr{V}}$, and by the inequality in $(3.11)$ we have

$$
c \varlimsup_{n \rightarrow \infty}\left|E_{n} u_{n}-u\right|_{\mathscr{V}}^{r} \leq \varlimsup_{n \rightarrow \infty}\left(\mathcal{A}\left(E_{n} u_{n}\right), E_{n} u_{n}-u\right)_{\mathscr{V}} \leq 0,
$$

i.e., $E_{n} u_{n} \rightarrow u$ in $\mathscr{V}$, along a subsequence.

Remark. Note that for the previous proof to hold true, the compatibility condition A6 in (3.5) can be replaced by the following weaker condition:

$$
\exists a>0 \text { such that } e^{-a \tau} S(\tau)\left(\mathscr{K} \cap \bar{B}_{\rho}(0 ; \mathscr{V})\right) \subset \mathscr{K} \quad \forall \tau \geq 0,
$$

where $\rho:=\sup _{n \in \mathbb{N}} R_{n}=\sup _{n \in \mathbb{N}}\left(\left|f_{n}\right|_{\mathscr{V}_{n}^{\prime}} / c_{n}\right)^{1 /\left(r_{n}-1\right)}$. Also, since $0 \in \mathscr{K}, \lambda \mathscr{K} \subset \mathscr{K}$ for $0 \leq \lambda \leq 1$; then if (3.13) holds, the same is true when " $\mathscr{K}$ " is replaced by " $\lambda \mathscr{K}$."

Copyright $@$ by SIAM. Unauthorized reproduction of this article is prohibited. 
Similarly, the conclusions of Lemma 2 hold true with the same replacements. This is later used in section 5.3.

Although the above consideration provides an approximation scheme to a solution of (3.6), this does not yet yield a solution to $\left(\mathrm{P}_{\mathrm{QVI}}\right)$. In order for this to be the case, " $L v$ " in (3.6) should be replaced by " $L u$." This requires characterizing $u$ as a more regular object. For that matter, extra assumptions need to be invoked (unless $\mathscr{K} \equiv \mathscr{V}$ where the Brézis-Bardos theorem holds; see [8] or Theorem 5.1 (Chapter III) in [37]). In this case, the following known result (see Corollary 9.1, Chapter 2 in [25]) can be established; the proof is included for the sake of completeness.

Corollary 3.4. Let the assumptions of Theorem 1 hold and suppose that $u$ satisfies

$$
u \in \mathscr{K}(u):(L v+\mathcal{A}(u)-f, v-u)_{\mathscr{V}} \geq 0 \quad \forall v \in \mathscr{K}(u) \cap \mathscr{D}\left(L ; \mathscr{V}^{\prime}\right),
$$

and $u \in \mathscr{D}\left(L ; \mathscr{V}^{\prime}\right)$. Then $u$ also satisfies

$$
(L u+\mathcal{A}(u)-f, v-u)_{\mathscr{V}} \geq 0 \quad \forall v \in \mathscr{K}(u) .
$$

Proof. Let $w \in \mathscr{K}(u) \cap \mathscr{D}\left(L ; \mathscr{V}^{\prime}\right)$, and let $w_{\lambda}=\lambda w+(1-\lambda) u$ with $0<\lambda<1$. Then $w_{\lambda} \in \mathscr{K}(u) \cap \mathscr{D}\left(L ; \mathscr{V}^{\prime}\right)$ (because of the assumption $u \in \mathscr{D}\left(L ; \mathscr{V}^{\prime}\right)$ ). Using $v=w_{\lambda}$ in (3.14), then dividing by $\lambda$, and subsequently taking the limit $\lambda \downarrow 0$, we observe that $u \in \mathscr{K}(u) \cap \mathscr{D}\left(L ; \mathscr{V}^{\prime}\right)$ satisfies

$$
(L u+\mathcal{A}(u)-f, w-u)_{\mathscr{V}} \geq 0 \quad \forall w \in \mathscr{K}(u) \cap \mathscr{D}\left(L ; \mathscr{V}^{\prime}\right) .
$$

By the compatibility condition A6, for arbitrary $v \in \mathscr{K}(u)$ there is a sequence $\left\{v_{k}\right\} \subset$ $\mathscr{K}(u) \cap \mathscr{D}\left(L ; \mathscr{V}^{\prime}\right)$ such that $v_{k} \rightarrow v$ in $\mathscr{V}$. Using $w=v_{k}$ in the above inequality and taking the limit as $k \rightarrow \infty$, we obtain (3.15).

Remark. Note that, as in Theorem 1, assumption A6 may be relaxed by the assumption described in the remark at the end of the proof of Theorem 1. The last step of the proof in Corollary 3.4 reads in this case: for arbitrary $v \in \mathscr{K}(u), \exists \lambda>0$ : $\lambda v \in\left(\lambda \mathscr{K}(u) \cap \bar{B}_{\rho}(0 ; \mathscr{V})\right)$; then $\exists\left\{\tilde{v}_{k}\right\} \subset \lambda \mathscr{K}(u) \cap \mathscr{D}\left(L ; \mathscr{V}^{\prime}\right)$ such that $\frac{\tilde{v}_{k}}{\lambda} \rightarrow v$ (and $\left.\left\{\tilde{v}_{k} / \lambda\right\} \subset \mathscr{K}(u) \cap \mathscr{D}\left(L ; \mathscr{V}^{\prime}\right)\right)$ in $\mathscr{V}$. Using $w=\frac{\tilde{v}_{k}}{\lambda}$ in (3.16) and taking the limit, the desired result holds.

Suppose that $\mathscr{V}=L^{p}(\mathrm{I} ; V), \mathcal{A}(v)(t)=A(v(t))$ as in Proposition 2.1 for all $v \in \mathscr{V}$ and $t \in \mathrm{I}$ with $|\mathrm{I}|<\infty,(f, v)_{\mathscr{V}}:=\int_{\mathrm{I}}(f(t), v(t))_{V} \mathrm{~d} t$ for $f \in L^{p^{\prime}}\left(\mathrm{I} ; V^{\prime}\right)$, and $\mathscr{K}(v)=$ $\{w \in \mathscr{V}: w(t) \in \mathbf{K}(v, t)$ a.e. $t \in \mathrm{I}\}$, where $\mathbf{K}: \mathscr{V} \times \mathrm{I} \rightarrow 2^{V}$, and for each $w \in \mathscr{V}$ and $t \in \mathrm{I}, 0 \in \mathbf{K}(w, t)$, with the latter a closed, convex subset of $V$. Then, in this case, if $u \in \mathscr{K}(u) \cap \mathscr{D}\left(L ; \mathscr{V}^{\prime}\right)$ satisfies (3.15), then equivalently it satisfies

$$
((L u)(t)+\mathcal{A}(u)(t)-f(t), v-u(t))_{V} \geq 0 \quad \forall v \in \mathbf{K}(u, t) \text { a.e. } t \in \mathrm{I} .
$$

This is sometimes called the instantaneous form of (3.15) and it is used later in our numerical tests.

4. Regularity of solutions of VIs. Throughout this section we assume that the map $v \mapsto \mathscr{K}(v)$ is constant and equal to some closed and convex $\mathscr{K} \subset 2^{\mathscr{V}}$ such that $0 \in \mathscr{K}$. From Corollary 3.4, we know that if $u$ satisfies (3.14) and $u$ is more regular (i.e., $\left.u \in \mathbf{d}\left(L ; \mathscr{V}^{\prime}\right)\right)$, then $u$ solves $\left(\mathrm{P}_{\mathrm{QVI}}\right)$. In what follows, we want to address the following question:

- Which conditions on $\mathscr{K} \subset \mathscr{V}, S(\tau)$, and $f \in \mathscr{V}^{\prime}$ guarantee that $u \in \mathscr{D}\left(L^{m} ; \mathscr{V}\right)$ for $m \geq 1$ ? 
An answer for the case $m=1$ was given by Brézis and Lions in their seminal work on parabolic VIs (see [7] and [25]). In this case, if

a. $\mathcal{A}: \mathscr{V} \rightarrow \mathscr{V}^{\prime}$ satisfies hypothesis $\mathrm{H} 1$ with $r=2$;

b. $S(\tau) \mathcal{A}(v)=\mathcal{A}(S(\tau) v) \forall v \in \mathscr{V}, \tau \geq 0$;

c. $\exists \alpha>0: S(\tau) v+S^{*}(\tau) v-S^{*}(\tau) S(\tau) v+(\alpha-1) v \in\left(\alpha+O\left(\tau^{2}\right)\right) \mathscr{K} \forall v \in \mathscr{K}$;

d. $f \in \mathscr{D}\left(L ; \mathscr{V}^{\prime}\right)$.

Then it can be shown that the solution to (3.14) satisfies $u \in \mathscr{D}(L ; \mathscr{V})$ (for a proof, see Proposition 7.3, Chapter III in [37] or [7]) and solves $\left(\mathrm{P}_{\mathrm{QVI}}\right)$ (as proven in Corollary 3.4). Although we are assuming in this section that $\mathscr{K}$ is constant, it should be noted that in the case when it is not, and when the map $v \mapsto \mathscr{K}(v)$ satisfies that every evaluation $\mathscr{K}(v)$ fulfills conditions a-d, then the solution to (3.14) satisfies $u \in \mathscr{D}(L ; \mathscr{V})$ and hence solves $\left(\mathrm{P}_{\mathrm{QVI}}\right)$. A detailed analysis for a nonconstant $v \mapsto \mathscr{K}(v)$ that determines the regularity required to have a solution to $\left(\mathrm{P}_{\mathrm{QVI}}\right)$ is given later in section 5.3.

In order to extend the result to $m>1$, stronger conditions are required as we shall see in the following. For the sake of simplicity, we also assume in this section that $S(\tau)$ is also a semigroup of contractions on $\mathscr{V}$.

TheOREM 2. Let $\mathcal{A}: \mathscr{V} \rightarrow \mathscr{V}^{\prime}$ satisfy hypotheses $\mathrm{H} 1$ with $r=2$, i.e.,

$$
(\mathcal{A}(v)-\mathcal{A}(w), v-w)_{\mathscr{V}} \geq c|v-w|_{\mathscr{V}}^{2} \forall v, w, \in \mathscr{V}
$$

$\mathrm{H} 2$ and $\mathrm{H} 3$, and let $S(\tau)$ and $\mathscr{K}$ satisfy $\mathrm{A} 6$. In addition, suppose

i. for all $v, w \in \mathscr{V}$ and $\tau \geq 0$ it holds that

$$
\left(\mathcal{A}(v),\left(S^{*}(\tau)-I\right) w\right)_{\mathscr{V}}=(\mathcal{A}((S(\tau)-I) v), w)_{\mathscr{V}} .
$$

ii. there exists $0<R \leq \infty$ such that for all $v \in \mathscr{K} \cap \bar{B}_{R}(0 ; \mathscr{V})$ and for all $\tau \in\left(0, \tau_{1}\right)$,

$$
\left(-\left(S^{*}(\tau)-I\right)^{m}(S(\tau)-I)^{m}+\alpha\right) v \in(\alpha+\theta(\tau)) \mathscr{K}
$$

for some $\tau_{1}>0,2 \leq m \in \mathbb{N}, \alpha>0$, and $0 \leq \theta(\tau)=O\left(\tau^{2 m}\right)$ as $\tau \downarrow 0$;

iii. $f \in \mathscr{D}\left(L^{m} ; \mathscr{V}^{\prime}\right):=\left\{g \in \mathscr{D}\left(L^{m-1} ; \mathscr{V}^{\prime}\right): \lim _{\tau \downarrow 0} \frac{(S(\tau)-I)}{\tau} L g\right.$ exists $\}$ and $|f|_{\mathscr{V}^{\prime}} \leq c R$.

Then, the solution to the problem

$$
\text { Find } u \in \mathscr{K}:(L v+\mathcal{A}(u)-f, v-u)_{\mathscr{V}} \geq 0 \quad \forall v \in \mathscr{K} \cap \mathscr{D}\left(L ; \mathscr{V}^{\prime}\right)
$$

satisfies $u \in \mathscr{D}\left(L^{m} ; \mathscr{V}\right)$ and

$$
(L u+\mathcal{A}(u)-f, v-u)_{\mathscr{V}} \geq 0 \quad \forall v \in \mathscr{K} .
$$

Proof. Let $\mathscr{V}_{n}=\mathscr{V}, \mathcal{A}_{n}=\mathcal{A}, f_{n}=f, \mathscr{K}_{n}(\cdot)=\mathscr{K}, P_{n}=E_{n}=I$, and $L_{n}=$ $(I-S(1 / n)) /(1 / n)$ for $n \in \mathbb{N}$. Then, by Theorem 1 , there exists a sequence $\left\{u_{n}\right\}$ such that for each $n \in \mathbb{N}, u_{n} \in \mathscr{K}$, and

$$
\left(\mathcal{B}_{n}\left(u_{n}\right)-f, v-u_{n}\right)_{\mathscr{V}} \geq 0 \quad \forall v \in \mathscr{K},
$$

where $\mathcal{B}_{n}(\cdot):=L_{n}(\cdot)+\mathcal{A}(\cdot), u_{n} \rightarrow u$ in $\mathscr{V}$ (along a subsequence), and $u \in \mathscr{K}$ solves

Find $u \in \mathscr{K}:(L v+\mathcal{A}(u)-f, v-u)_{\mathscr{V}} \geq 0 \quad \forall v \in \mathscr{K} \cap \mathscr{D}\left(L ; \mathscr{V}^{\prime}\right)$.

Since $0 \in \mathscr{K}, L_{n}$ is monotone, and $\mathcal{A}$ is uniformly monotone (hypothesis H1), we have that $\mathcal{B}_{n}$ is also uniformly monotone and $\left(\mathcal{B}_{n}(w), w\right) \geq c|w|_{\mathscr{V}}^{2}$. Hence, taking $v=0$ in 
(4.4), we have that $\left|u_{n}\right|_{\mathscr{V}} \leq|f|_{\mathscr{V}^{\prime}} / c \leq R$, since $|f|_{\mathscr{V}^{\prime}} \leq c R$ (because of iii). Therefore, $u_{n} \in \mathscr{K} \cap \bar{B}_{R}(0 ; \mathscr{V})$ for all $n \in \mathbb{N}$ and, by ii, for each $\tau \in\left(0, \tau_{1}\right)$ there exists a $v \in \mathscr{K}$ such that

$$
(\alpha+\theta(\tau)) v=-\left(S^{*}(\tau)-I\right)^{m}(S(\tau)-I)^{m} u_{n}+\alpha u_{n} .
$$

Therefore, multiplying (4.4) by $\alpha+\theta(\tau) \geq 0$, we obtain

$$
\left(\mathcal{B}_{n}\left(u_{n}\right)-f,-\left(S^{*}(\tau)-I\right)^{m}(S(\tau)-I)^{m} u_{n}-\theta(\tau) u_{n}\right)_{\mathscr{V}} \geq 0
$$

or, equivalently,

$$
\begin{aligned}
\left(\mathcal{B}_{n}\left(u_{n}\right),\left(S^{*}(\tau)-I\right)^{m}(S(\tau)-I)^{m} u_{n}\right) \mathscr{V}+\theta(\tau)\left(\mathcal{B}_{n}\left(u_{n}\right), u_{n}\right)_{\mathscr{V}} \\
\leq\left((S(\tau)-I)^{m} f,(S(\tau)-I)^{m} u_{n}\right)_{\mathscr{V}}+\theta(\tau)\left(f, u_{n}\right)_{\mathscr{V}}
\end{aligned}
$$

Since $L_{n}=(I-S(1 / n)) /(1 / n)$, it commutes with $(S(\tau)-I)$ and hence $\left(L_{n}(v)\right.$, $\left.\left(S^{*}(\tau)-I\right) w\right)=\left(L_{n}((S(\tau)-I) v), w\right)$, i.e., $L_{n}$ satisfies the same condition as $\mathcal{A}$ in i. Therefore, by applying successively hypothesis i, we obtain

$$
\begin{aligned}
\left(\mathcal{B}_{n}\left((S(\tau)-I)^{m} u_{n}\right)\right. & \left.,\left((S(\tau)-I)^{m} u_{n}\right)\right)_{\mathscr{V}}+\theta(\tau)\left(\mathcal{B}_{n}\left(u_{n}\right), u_{n}\right)_{\mathscr{V}} \\
\leq & \left((S(\tau)-I)^{m} f,(S(\tau)-I)^{m} u_{n}\right)_{\mathscr{V}}+\theta(\tau)\left(f, u_{n}\right)_{\mathscr{V}}
\end{aligned}
$$

Using the uniform monotonicity of $\mathcal{B}_{n}$ and that $\theta(\tau) \geq 0$, we have that

$$
c\left|(S(\tau)-I)^{m} u_{n}\right|_{\mathscr{V}}^{2} \leq\left|(S(\tau)-I)^{m} f\right|_{\mathscr{V}^{\prime}}\left|(S(\tau)-I)^{m} u_{n}\right|_{\mathscr{V}}+\theta(\tau)|f|_{\mathscr{V}^{\prime}}\left|u_{n}\right|_{\mathscr{V}} .
$$

Dividing by $\tau^{2 m}$ and since $f \in \mathscr{D}\left(L^{m} ; \mathscr{V}^{\prime}\right)$ and $\theta(\tau)=O\left(\tau^{2 m}\right)$, we observe that (for some $M>0$ )

$$
c \sup _{\tau \in\left(0, \tau_{1}\right)}\left|\frac{(S(\tau)-I)^{m}}{\tau^{m}} u_{n}\right|_{\mathscr{V}}^{2} \leq C_{f} \sup _{\tau \in\left(0, \tau_{1}\right)}\left|\frac{(S(\tau)-I)^{m}}{\tau^{m}} u_{n}\right|_{\mathscr{V}}+M|f|_{\mathscr{V}^{\prime}}\left|u_{n}\right|_{\mathscr{V}},
$$

where $C_{f}:=\sup _{\tau \in\left(0, \tau_{1}\right)}\left|\frac{(S(\tau)-I)^{m}}{\tau^{m}} f\right|_{\mathscr{V}^{\prime}}<\infty$. The latter holds true due to

$$
\lim _{\tau \downarrow 0}\left(\frac{S(\tau)-I}{\tau}\right)^{m} f=L^{m} f
$$

and the fact that $\mathscr{D}\left(L^{m} ; \mathscr{V}^{\prime}\right)=\left\{g \in \mathscr{D}\left(L^{m-1} ; \mathscr{V}^{\prime}\right): L^{m-1} g \in \mathscr{D}\left(L ; \mathscr{V}^{\prime}\right)\right\}$ is equivalently characterized as

$$
\mathscr{D}\left(L^{m} ; \mathscr{V}^{\prime}\right)=\left\{g \in \mathscr{V}^{\prime}: \lim _{\tau \downarrow 0}\left(\frac{S(\tau)-I}{\tau}\right)^{m} g \text { exists }\right\},
$$

as is proven in [6]. Then, since $\left|u_{n}\right|_{\mathscr{V}} \leq|f|_{\mathscr{V}^{\prime}} / c$ and $\left|\frac{(S(\tau)-I)^{m}}{\tau^{m}} u_{n}\right|_{\mathscr{V}} \leq \frac{2^{m}}{\tau_{1}^{m}}\left|u_{n}\right|_{\mathscr{V}}$ for $\tau \geq \tau_{1}$ (due to $|S(\tau)|_{\mathscr{L}(\mathscr{V}, \mathscr{V})} \leq 1$ for all $\tau>0$ ), we obtain

$$
\sup _{n \in \mathbb{N}}\left(\sup _{\tau>0}\left|\frac{(S(\tau)-I)^{m}}{\tau^{m}} u_{n}\right|_{\mathscr{V}}\right)<\infty .
$$

For a fixed $n \in \mathbb{N}$, an application of the generalized Landau-Kolmogorov inequality for semigroups of contractions (see [11]) also yields

$$
\sup _{n \in \mathbb{N}}\left(\sup _{\tau>0}\left|\frac{(S(\tau)-I)^{j}}{\tau^{j}} u_{n}\right|_{\mathscr{V}}\right)<\infty \text { for } j=1,2, \ldots, m-1 .
$$

Copyright $@$ by SIAM. Unauthorized reproduction of this article is prohibited. 
From elementary properties of $C_{0}$-semigroups (see, for example, [31]), we have that $\int_{0}^{\tau} S(t) u_{n} \mathrm{~d} t \in \mathscr{D}(L ; \mathscr{V}), \lim _{\tau \downarrow 0} \frac{1}{\tau} \int_{0}^{\tau} S(t) u_{n} \mathrm{~d} t=u_{n}$, and $-L\left(\frac{1}{\tau} \int_{0}^{\tau} S(t) u_{n} \mathrm{~d} t\right)=$ $\frac{S(\tau)-I}{\tau} u_{n}$. Since the right-hand sides above are bounded and $\mathscr{V}$ is reflexive, we have that

$$
\frac{1}{\tau_{i}} \int_{0}^{\tau_{i}} S(t) u_{n} \mathrm{~d} t \rightarrow u_{n} \quad \text { and } \quad-L\left(\frac{1}{\tau_{i}} \int_{0}^{\tau_{i}} S(t) u_{n} \mathrm{~d} t\right) \rightarrow y,
$$

both in $\mathscr{V}$, as $i \rightarrow \infty$ for some sequence such $\tau_{i} \downarrow 0$. Therefore, as $-L$ is closed (for being the infinitesimal generator of $S(\tau))$ and $\mathscr{V}$ is a Banach space, $-L$ is weakly closed and thus $u_{n} \in \mathscr{D}(L ; \mathscr{V})$ as well as $-L\left(\frac{1}{\tau_{i}} \int_{0}^{\tau_{i}} S(t) u_{n} \mathrm{~d} t\right) \rightarrow-L u_{n}$.

Suppose now that $u_{n} \in \mathscr{D}\left(L^{q-1} ; \mathscr{V}\right)$ for some $1 \leq q-1 \leq m-1$. Then, $w_{n}^{\tau}:=$ $\left(\frac{S(\tau)-I}{\tau}\right)^{q-1} u_{n}$ satisfies $\lim _{\tau \downarrow 0} w_{n}^{\tau}=(-L)^{q-1} u_{n}$ (see [6]). Hence, $\int_{0}^{\tau} S(t) w_{n}^{\tau} \mathrm{d} t \in$ $\mathscr{D}(L ; \mathscr{V})$, and

$$
\left|\frac{1}{\tau} \int_{0}^{\tau} S(t) w_{n}^{\tau} \mathrm{d} t-\frac{1}{\tau} \int_{0}^{\tau} S(t)(-L)^{q-1} u_{n} \mathrm{~d} t\right|_{\mathscr{V}} \leq\left|w_{n}^{\tau}-(-L)^{q-1} u_{n}\right|_{\mathscr{V}}
$$

implies that $\lim _{\tau \downarrow 0} \frac{1}{\tau} \int_{0}^{\tau} S(t) w_{n}^{\tau} \mathrm{d} t=(-L)^{q-1} u_{n}$. Also, $-L\left(\frac{1}{\tau} \int_{0}^{\tau} S(t) w_{n}^{\tau} \mathrm{d} t\right)=\frac{S(\tau)-I}{\tau} w_{n}^{\tau}$ $=\left(\frac{S(\tau)-I}{\tau}\right)^{q} u_{n}$, with the latter term bounded by (4.7) because of $2 \leq q \leq m$. Therefore,

$$
\frac{1}{\tau_{i}} \int_{0}^{\tau_{i}} S(t) w_{n}^{\tau_{i}} \mathrm{~d} t \rightarrow(-L)^{q-1} u_{n} \quad \text { and } \quad-L\left(\frac{1}{\tau_{i}} \int_{0}^{\tau_{i}} S(t) w_{n}^{\tau_{i}} \mathrm{~d} t\right) \rightarrow z
$$

both in $\mathscr{V}$, as $i \rightarrow \infty$ for some sequence $\tau_{i} \downarrow 0$. Again using the weak-closedness of $(-L)$, we have $(-L)^{q-1} u_{n} \in \mathscr{D}(L ; \mathscr{V})$ or, equivalently, $u_{n} \in \mathscr{D}\left(L^{q} ; \mathscr{V}\right)$ and $z=$ $(-L)(-L)^{q-1} u_{n}=(-L)^{q} u_{n}$.

Therefore, by induction, since $u_{n} \in \mathscr{D}(L ; \mathscr{V})$, we have $L u_{n}, L^{2} u_{n}, \ldots, L^{m-1} u_{n} \in$ $\mathscr{D}(L ; \mathscr{V})$, i.e., $u_{n} \in \mathscr{D}\left(L^{m} ; \mathscr{V}\right)$. Consequently, by $(4.6)$ and $(4.7)$, there exists a constant $C>0$ such that

$$
\left|(-L)^{j} u_{n}\right|_{\mathscr{V}} \leq C \quad \forall n \in \mathbb{N} \text { and } j=1,2, \ldots, m .
$$

Thus, using the weak-closedness of $-L$ and the reflexivity of $\mathscr{V}$, as before we have that if $(-L)^{k} u_{n} \rightarrow(-L)^{k} u$, then $u \in \mathscr{D}(L ; \mathscr{V})$ and $(-L)^{k+1} u_{n} \rightarrow(-L)^{k+1} u$ for $k=$ $0,1, \ldots, m-1$ ( where $(-L)^{0} \equiv I$ ), respectively. Since $u_{n} \rightarrow u$ along a subsequence (by Theorem 1 and where $u$ is the solution to (4.2)) we have that $L u, L^{2} u, \ldots, L^{m-1} u \in$ $\mathscr{D}(L ; \mathscr{V})$, that is, $u \in \mathscr{D}\left(L^{m} ; \mathscr{V}\right)$. Finally, Corollary 3.4 proves that $u$ solves $(4.3)$.

Remark. The previous theorem also holds in the case $m=1$, but the conditions required by its hypotheses are stronger than those invoked by Brézis and Lions (see [7] and section 9.2, Chapter 2 of [25]). In fact, condition ii, for the case $m=1$, is simply proven to hold for $R=\infty, \mathscr{V}=L^{p}(\mathrm{I} ; V), \mathcal{K}=\{v \in \mathscr{V}: v(t) \in \mathbf{K}$ a.e. $\}$, i.e., when $\mathbf{K}$ does not depend on $t$ (see [7, 25]). However, for $m \geq 2$ condition ii fails to hold even in the simplest cases if $R=\infty$.

4.1. Applications of the regularity theorem. Condition i is satisfied for linear "translation invariant" operators $\mathcal{A}: \mathscr{V} \rightarrow \mathscr{V}^{\prime}$; i.e., if $\mathcal{A}(v+h w)=\mathcal{A}(v)+h \mathcal{A}(w)$ for all $h \in \mathbb{R}, v, w \in \mathscr{V}$, and $S(\tau) \mathcal{A}(v)=\mathcal{A}(S(\tau) v)$ for all $\tau \geq 0$ and $v \in \mathscr{V}$. For example, consider $\mathscr{V}=L^{2}(\mathrm{I} ; V)$ and $\mathscr{H}=L^{2}(\mathrm{I} ; H)$, where $\left(V, H, V^{\prime}\right)$ is a Gelfand triple, with $S(\tau)$ as in Example 2.3. If $A: V \rightarrow V^{\prime}$ is a linear operator, then 
$(\mathcal{A} v)(t):=A v(t)$ with $t \in \mathrm{I}$ and $\mathrm{i}$ is satisfied. If $V=H_{0}^{1}(\Omega), H=L^{2}(\Omega)$, then $A$ can be taken as $-\Delta$, for instance.

Example 4.1. Let $\mathscr{K}=\mathscr{V}$. In this case, condition ii is trivially satisfied with $R=\infty$, for any $m \in \mathbb{N}$ and any $\alpha>0$. Moreover, the VI in (4.3) becomes an equation in $\mathscr{V}^{\prime}$; i.e., if $f \in \mathscr{D}\left(L^{m} ; \mathscr{V}^{\prime}\right)$, we observe that there exists $u \in \mathscr{D}\left(L^{m} ; \mathscr{V}\right)$ satisfying

$$
L u+\mathcal{A}(u)-f=0
$$

in the sense of $\mathscr{V}^{\prime}$. In this case, $f \in \mathscr{D}\left(L^{m} ; \mathscr{V}^{\prime}\right)$ implies $u \in \mathscr{D}\left(L^{m} ; \mathscr{V}\right)$ without additional assumptions on $f$ and on $S(\tau)$.

Example 4.2. Let $\mathscr{V}=L^{2}(\mathrm{I} ; V)$ with $\mathrm{I}=(0, \infty)$ and $\left(V, H, V^{\prime}\right)$ a Gelfand triple with $V$ a reflexive separable Banach space, $k_{i} \in L^{2}\left(\mathrm{I} ; V^{\prime}\right), h_{i}>0$ for $i=1,2, \ldots, N$, and let $\mathscr{K}$ be given by

$$
\mathscr{K}=\left\{v \in \mathscr{V}: \int_{\mathrm{I}}\left|\left(k_{i}(t), v(t)\right)_{V}\right| \mathrm{d} t \leq h_{i} \text { for } i=1,2, \ldots, N\right\} .
$$

We have that $\mathscr{K}$ is closed since the map $v \mapsto \int_{\mathrm{I}}\left|\left(k_{i}, v\right)_{V}\right| \mathrm{d} t$ is continuous in $\mathscr{V}$ and convex since $\int_{\mathrm{I}}\left|\left(k_{i}, \lambda v_{1}+(1-\lambda) v_{2}\right)_{V}\right| \mathrm{d} t \leq \lambda \int_{\mathrm{I}}\left|\left(k_{i}, v_{1}\right)_{V}\right| \mathrm{d} t+(1-\lambda) \int_{\mathrm{I}}\left|\left(k_{i}, v_{2}\right)_{V}\right| \mathrm{d} t$ for $\lambda \in[0,1]$ and $0 \in \mathscr{K}$.

Let $S(\tau)$ be as in Example 2.3. Then for $i=1,2, \ldots, N$, if there exists $a_{i}>0$ such that $\int_{\mathrm{I}}\left|\left(k_{i}(t+\tau), v(t)\right)_{V}\right| \mathrm{d} t \leq e^{a_{i} \tau} \int_{\mathrm{I}}\left|\left(k_{i}(t), v(t)\right)_{V}\right| \mathrm{d} t$ for all $v \in \mathscr{K}$ and $\tau \geq 0$, then the compatibility condition $e^{-a \tau} S(\tau) \mathscr{K} \subset \mathscr{K}$ is satisfied with $a=\max \left(a_{i}\right)$. For example, consider $k_{i}(t)=e^{-\eta_{i} t} \varphi$, with $\eta_{i}>0$ and $\varphi \in V^{\prime}$.

Suppose that $\alpha=6$ and $\theta(\tau)=0$. Then, since $\left(S^{*}(\tau) v\right)(t)=v(t+\tau)$ we have that $S^{*}(\tau) S(\tau)=I$ and further

$$
\begin{aligned}
w & :=\left(-\left(S^{*}(\tau)-I\right)^{2}(S(\tau)-I)^{2}+\alpha\right) v \\
& =\left(-\left(S^{*}(\tau)\right)^{2}-(S(\tau))^{2}+4\left(S^{*}(\tau)+S(\tau)\right)\right) v .
\end{aligned}
$$

Hence, $|w|_{\mathscr{V}} \leq 10|v|_{\mathscr{V}}$ and if $|v|_{\mathscr{V}} \leq \frac{6 \min \left(h_{i}\right)}{10 \max \left(\left|k_{i}\right|_{\mathscr{V}^{\prime}}\right)}$, we obtain

$$
\int_{\mathrm{I}}\left|\left(k_{i}(t), w(t)\right)_{V}\right| \mathrm{d} t \leq\left|k_{i}\right|_{\mathscr{V}^{\prime}}|w|_{\mathscr{V}} \leq 6 h_{i} .
$$

Consequently, if $v \in \mathscr{K} \cap\left\{v \in \mathscr{V}:|v|_{\mathscr{V}} \leq R\right\}$, with $R=\frac{6 \min \left(h_{i}\right)}{10 \max \left(\left|k_{i}\right|_{\mathscr{V}^{\prime}}\right)}$, we then observe

$$
\left(-\left(S^{*}(\tau)-I\right)^{2}(S(\tau)-I)^{2}+6\right) v \in 6 \mathscr{K} .
$$

Finally, if $f \in \mathscr{D}\left(L^{2} ; \mathscr{V}^{\prime}\right)$ and $|f|_{\mathscr{V}^{\prime}} \leq c R$ with the previously defined $R$, the hypotheses of Theorem 2 are met and $u \in \mathscr{D}(L ; \mathscr{V}) \cap \mathscr{K}$, the solution to the problem

$$
\text { Find } u \in \mathscr{K} \cap \mathscr{D}\left(L ; \mathscr{V}^{\prime}\right):(L u+\mathcal{A}(u)-f, v-u)_{\mathscr{V}} \geq 0 \quad \forall v \in \mathscr{K},
$$

which also satisfies $u \in \mathscr{D}\left(L^{2} ; \mathscr{V}\right)$.

5. Applications of the general theory. Let $\mathrm{I}=(0, T)$ with $0<T \leq \infty$ and $\mathscr{V}=L^{p}(\mathrm{I} ; V)$, where $2 \leq p<\infty$ and $V$ is a reflexive separable Banach space, and let $\left(V, H, V^{\prime}\right)$ be a Gelfand triple with some separable Hilbert space $H$. Since $I$ is $\sigma$-finite, $\mathscr{V}^{\prime}=L^{p^{\prime}}\left(\mathrm{I} ; V^{\prime}\right)$ (see Theorem 1.5, Chapter III in [37]) and we have the Gelfand triple $\left(\mathscr{V}, \mathscr{H}, \mathscr{V}^{\prime}\right)($ if $T<\infty)$, where $\mathscr{H}:=L^{2}(\mathrm{I} ; H)$. In the case $T=\infty$, the previous holds true for $p=p^{\prime}=2$. 
5.1. Characterization of $\mathscr{D}(L ; \mathscr{V}), \mathscr{D}(L ; \mathscr{H})$, and $\mathscr{D}\left(L ; \mathscr{V}^{\prime}\right)$. This characterization is based on a proof similar to the one in [17, Chapter XIX] for translations on $L^{p}(\mathrm{I} ; \mathbb{C})$ spaces. If $f \in \mathscr{V}^{\prime}$, let $S(t)$ be the $C_{0}$-semigroup over $\mathscr{V}^{\prime}$ defined by

$$
(S(\tau) f)(t)= \begin{cases}f(t-\tau), & \tau<t<T \\ 0 & \text { otherwise }\end{cases}
$$

Clearly, $S(0)=I, S\left(\tau_{1}+\tau_{2}\right)=S\left(\tau_{1}\right) S\left(\tau_{2}\right)$ and $\lim _{\tau \downarrow 0} S(\tau) f=f$ for $f \in \mathscr{V}^{\prime}$. We also observe that $S(\tau)$ is a $C_{0}$-semigroup of contractions over $\mathscr{V}, \mathscr{H}$, and $\mathscr{V}^{\prime}$. Denote the infinitesimal generator of $S(t)$ over $\mathscr{V}^{\prime}$ by $-L$ and its domain by $\mathscr{D}\left(L ; \mathscr{V}^{\prime}\right)$. If $f \in \mathscr{D}\left(L ; \mathscr{V}^{\prime}\right)$, then

$$
\lim _{\tau \downarrow 0} \frac{S(\tau) f-f}{\tau} \text { exists in } \mathscr{V}^{\prime}
$$

and is defined as $-L f:=g$. Since $f \in L_{l o c}^{1}\left(\mathrm{I} ; V^{\prime}\right) \subset \mathscr{V}^{\prime}$ for $0<\tau \leq \alpha \leq \beta<T$, we have

$$
\frac{1}{\tau} \int_{\alpha-\tau}^{\alpha} f(t) \mathrm{d} t-\frac{1}{\tau} \int_{\beta-\tau}^{\beta} f(t) \mathrm{d} t=\int_{\alpha}^{\beta} \frac{f(t-\tau)-f(t)}{\tau} \mathrm{d} t \rightarrow \int_{\alpha}^{\beta} g(t) \mathrm{d} t
$$

as $\tau \downarrow 0$. Also, since $f \in L_{\text {loc }}^{1}\left(\mathrm{I} ; V^{\prime}\right)$, almost all $\gamma \in \mathrm{I}$ are Lebesgue points, i.e., $\lim _{\tau \downarrow} \frac{1}{\tau} \int_{\gamma}^{\gamma+\tau} f(t) \mathrm{d} t=f(\gamma)$ for almost all $\gamma \in[0, \infty)$ (see Proposition 1.2.4 in [2]). Hence, if $\alpha \in[0, \infty)$ is a Lebesgue point, for almost all $\beta \in[0, \infty)$, we have

$$
f(\alpha)-f(\beta)=\int_{\alpha}^{\beta} g(t) \mathrm{d} t .
$$

Since $g \in L_{l o c}^{1}\left(\mathrm{I} ; V^{\prime}\right) \subset \mathscr{V}^{\prime}$, the map $\alpha \mapsto \int_{\alpha}^{\beta} g(t) \mathrm{d} t$ is continuous for $\alpha \in[0, \infty)$. This fact follows from applying Lebesgue's bounded convergence theorem to $\int_{I} \chi_{(\alpha, \beta)}(t) g(t)$ $\mathrm{d} t$. Hence, we define $f(0)$ as $f(\beta)+\int_{0}^{\beta} g(t) \mathrm{d} t$ for some $\beta$ which is a Lebesgue point, and redefine $f$ on the measure-zero-set of non-Lebesgue points, which yields $f(t)=f(0)+$ $\int_{0}^{t}(-g(s)) \mathrm{d} s$ for $t \in[0, \infty)$. Also, $\left.\left|\frac{1}{\tau}\left(\int_{0}^{\tau}|f|_{V^{\prime}}^{p^{\prime}}\right)^{1 / p^{\prime}}-\right| g\right|_{L^{p^{\prime}}\left((0, \tau) ; V^{\prime}\right)}|\leq| \frac{S(\tau) f-f}{\tau}-\left.g\right|_{\mathscr{V}^{\prime}}$ and the right-hand side goes to zero as $\tau \downarrow 0$, which implies $f(0)=0$ and hence

$$
f(t)=\int_{0}^{t}(-g(s)) \mathrm{d} s
$$

Therefore, if $f \in \mathscr{D}\left(L ; \mathscr{V}^{\prime}\right)$, then $f(0)=0$ and $f \in A C^{1, r^{\prime}}\left(\mathrm{I} ; V^{\prime}\right)$, the space of $V^{\prime}$ valued, absolutely continuous functions whose strong derivatives $-g$ exist a.e. (we refer to the existence of $\lim _{h \rightarrow 0} \frac{f(t+h)-f(t)}{h}$ in $V^{\prime}$ ) and belong to $L^{r^{\prime}}\left(\mathrm{I} ; \mathscr{V}^{\prime}\right.$ ) (for a proof that absolutely continuous functions $f: \mathrm{I} \rightarrow \mathscr{V}^{\prime}$ are differentiable a.e., even if $|\mathrm{I}|=\infty$, see [2] and note that $\mathscr{V}^{\prime}$ satisfies the Radon-Nikodym property for being reflexive). Furthermore, if $f \in A C^{1, r^{\prime}}\left(\mathrm{I} ; V^{\prime}\right)$, then

$$
\frac{f(t-h)-f(t)}{h}=\frac{1}{h} \int_{0}^{h}(-g(t-\tau)) \mathrm{d} \tau=-\frac{1}{h} \int_{0}^{h} S(\tau) g(t) \mathrm{d} \tau
$$

and the right-hand side converges to $g$ in $\mathscr{V}^{\prime}$ as $h \downarrow 0$ (see Theorem 2.4. in [31]). From this, we infer the characterization

$$
\mathscr{D}\left(L ; \mathscr{V}^{\prime}\right):=\left\{f \in \mathscr{V}^{\prime}: f(0)=0, f \text { is absolutely continuous and } f^{\prime} \in \mathscr{V}^{\prime}\right\} ;
$$

in this sense $-L=-\frac{\mathrm{d}}{\mathrm{d} t}$. A similar characterization can be obtained for $\mathscr{D}(L ; \mathscr{V})$ and $\mathscr{D}(L ; \mathscr{H})$. 
5.2. Constant $\boldsymbol{v} \mapsto \mathscr{K}(\boldsymbol{v})$. We assume in this section that $\mathscr{V} \ni v \mapsto \mathscr{K}(v) \in$ $2^{\mathscr{V}}$ does not depend on $v$, i.e., $\mathscr{K}(v)=\mathscr{K}$ for all $v \in \mathscr{V}$, where

$$
\mathscr{K}=\{v \in \mathscr{V}: v(t) \in \mathbf{K}(t) \text { a.e. } t \in \mathrm{I}\} .
$$

Let $G \in \mathscr{L}(V, W)$, a bounded linear operator with domain in $V$ and image in $W$, and a Banach space of functions over some domain $\Omega \subset \mathbb{R}^{N}$ and range in $\mathbb{R}^{l}$ for which $f_{n} \rightarrow f$ in $W$ imply $f_{n}(\mathbf{x}) \rightarrow f(\mathbf{x})$ a.e. in $\mathbb{R}^{l}$ (along a subsequence) be given. Let $\varphi: I \rightarrow L^{\infty}(\Omega)$ with $\varphi(t, \mathbf{x}) \geq \nu>0$ a.e. $t \in \mathrm{I}, \mathbf{x} \in \Omega$, and

$$
\mathbf{K}(t):=\left\{y \in V:|(G y)(\mathbf{x})|_{\mathbb{R}^{l}} \leq \varphi(t, \mathbf{x}) \text { a.e. in } \Omega\right\} .
$$

Then, $0 \in \mathbf{K}(t)$ for all $t \in \mathrm{I}$ and hence $0 \in \mathscr{K}$. Each $\mathbf{K}(t)$ for $t \in \mathrm{I}$ is a convex subset of $V$, and hence $\mathscr{K}$ is a convex subset of $\mathscr{V}$, and if $\left\{v_{n}\right\} \subset \mathscr{K}$ such that $v_{n} \rightarrow v$ in $\mathscr{V}$, then by elementary properties of the Bochner integral, $G v_{n} \rightarrow G v$ on $L^{1}\left(\left(t_{0}, t_{1}\right) ; W\right)$ for any $0<t_{0}<t_{1}<\infty$. Hence $G v_{n}(t) \rightarrow G v(t)$ on $W$ a.e. (along a subsequence) and (for fixed $t)\left(G v_{n}(t)\right)(\mathbf{x}) \rightarrow(G v(t))(\mathbf{x})$ a.e. in $\mathbb{R}^{l}$ (also along a subsequence), i.e., $|(G v(t))(\mathbf{x})| \leq \varphi(t, \mathbf{x})$ holds a.e., which implies the closedness of $\mathscr{K}$.

The previous type of nonempty, closed, and convex set $\mathscr{K}$ is then determined only by $t \mapsto \varphi(t)$. Using this mapping we determine the compatibility condition of $S(\tau)$ and $\mathscr{K}$ and conditions to increase regularity of solutions to the VI.

The prototypical example is given by considering $V \equiv W_{0}^{1, r}(\Omega)$, with $\Omega$ some open domain in $\mathbb{R}^{N}, W \equiv L^{p}(\Omega)^{N}$, and $G \equiv \nabla$ the gradient, i.e.,

$$
\mathscr{K}=\left\{v \in L^{p}\left(\mathrm{I} ; W_{0}^{1, r}(\Omega)\right):|(\nabla w(t))(\mathbf{x})|_{\mathbb{R}^{l}} \leq \varphi(t, \mathbf{x}) \text { a.e. } t \in \mathrm{I}, \mathbf{x} \in \Omega\right\} .
$$

5.2.1. Compatibility and regularity conditions. Let $\mathrm{I}=(0, \infty)$ and $S(\tau)$ be the semigroup described in section 5.1. In this case $\left(S^{*}(\tau) f\right)(t)=f(t+\tau)$, and hence $S^{*}(\tau) S(\tau)=I$ for all $\tau \geq 0$ (i.e., $S(\tau)$ is pseudounitary in particular in $\mathscr{H}$ ). Since, as already stated, $0 \in \mathbf{K}(t)$ for all $t \geq 0$, which implies that $0 \in \mathscr{K}=\{v \in$ $\mathscr{V}: v(t) \in \mathbf{K}(t)$ a.e. $\}, S(\tau)$ and $\mathscr{K}$ are compatible (see [7]). Also, in order to observe that $u \in \mathscr{D}(L ; \mathscr{V})$, it is enough to satisfy

$$
\left(-\left(S^{*}(\tau)-I\right)(S(\tau)-I)+\alpha\right) v \in \alpha \mathscr{K}
$$

for each $v \in \mathscr{K}$, and $\tau \in\left(0, \tau_{1}\right)$ for some $\tau_{1}>0$. For $\alpha=2$, the previous relation reduces to $\left(S^{*}(\tau)+S(\tau)\right) v \in 2 \mathscr{K}$, which is satisfied, for example, if

$$
\begin{array}{rlrl}
\varphi(t+\tau, \mathbf{x})+\varphi(t-\tau, \mathbf{x}) & \leq 2 \varphi(t, \mathbf{x}) & & \text { if } \tau<t, \\
\varphi(t+\tau, \mathbf{x}) \leq 2 \varphi(t, \mathbf{x}) & & \text { if } 0 \leq t \leq \tau .
\end{array}
$$

A large variety of (nonconstant) mappings $\varphi: I \times \Omega \rightarrow(\nu, \infty)$ satisfies the previous set of equations. For example, for $\varphi(t, \mathbf{x})=\left(r_{1}-r_{2} e^{-r_{3} t}\right) h(\mathbf{x})$ with $r_{1}>r_{2} \geq 0$, $r_{3} \geq 0$, and $h(\mathbf{x}) \in L_{\nu}^{\infty}(\Omega)$, the previous equations reduce to

$$
\begin{aligned}
\cosh \left(r_{3} \tau\right) & \geq 1 & & \text { if } \tau<t, \\
-e^{-r_{3} \tau} & \leq \frac{r_{1}}{r_{2}} e^{r_{3} t}-2 & & \text { if } 0 \leq t \leq \tau .
\end{aligned}
$$

The first equation holds for all $\tau$ and the second holds if $r_{1} \geq r_{2}$.

If $\mathrm{I}=(0, T)$ with $T<\infty$, the compatibility condition (for $\varphi(t, \mathbf{x}) \geq \nu>0$ a.e. $t \in \mathrm{I}, \mathbf{x} \in \Omega)$ reduces to the growth condition: $\exists a>0: e^{-a \tau} \varphi(t-\tau, \mathbf{x}) \leq \varphi(t, \mathbf{x})$ for $t>\tau$. The regularity condition

$$
\left(-\left(S^{*}(\tau)-I\right)(S(\tau)-I)+\alpha\right) v \in\left(\alpha+M \tau^{2}\right) \mathscr{K},
$$


for $\alpha=2$, reduces to the three inequalities

$$
\begin{aligned}
\frac{\varphi(t+\tau, \mathbf{x})-\varphi(t, \mathbf{x})}{\tau} & \leq \frac{\varphi(t, \mathbf{x})}{\tau}+M \tau \varphi(t, \mathbf{x}) & & \text { if } t \in(0, \tau) \\
\frac{\varphi(t+\tau, \mathbf{x})-2 \varphi(t, \mathbf{x})+\varphi(t-\tau, \mathbf{x})}{\tau^{2}} & \leq M \varphi(t, \mathbf{x}) & & \text { if } t \in(\tau, T-\tau) \\
\frac{\varphi(t-\tau, \mathbf{x})-\varphi(t, \mathbf{x})}{\tau} & \leq M \tau \varphi(t, \mathbf{x}) & & \text { if } t \in(T-\tau, T) .
\end{aligned}
$$

Then, if $\varphi(t, \mathbf{x})=\phi(t) \eta(\mathbf{x})$, where $\eta \in L_{\nu}^{\infty}(\Omega)$, and if $\phi: \mathrm{I} \rightarrow(\delta, \infty)$, with $\delta>0$, is decreasing in some interval $(0, \epsilon)$, increasing in $(T-\epsilon, T)$, and the second order finite difference is uniformly bounded on $(\tau, T-\tau)$, then the above inequalities are satisfied for some $M>0$ and for $0<\tau<\epsilon$. Indeed, as an example consider $\phi(t)=$ $r 1-r 2(t(t-T))^{2 n}$ for $r_{1} \gg r_{2}>0$ and $n \in \mathbb{N}$. The case when the constraint set depends on the state variable is more complicated and is analyzed next.

5.3. Nonconstant $\boldsymbol{v} \mapsto \mathscr{K}(\boldsymbol{v})$. Now, suppose that $\mathscr{V} \ni v \mapsto \mathscr{K}(v) \in 2^{\mathscr{V}}$ depends on $v$ such that $\mathscr{K}(v)=\{v \in \mathscr{V}: v(t) \in \mathbf{K}(v, t)$ a.e. $t \in \mathrm{I}\}, \mathrm{I}=(0, T)$, with $T<\infty$, and

$$
\mathbf{K}(v, t):=\left\{y \in V:|(G y)(\mathbf{x})|_{\mathbb{R}^{l}} \leq(\Phi(v, t))(\mathbf{x}) \text { a.e. in } \Omega\right\},
$$

with $\Phi: \mathscr{V} \times \mathrm{I} \rightarrow L_{\nu}^{\infty}(\Omega)$. Consider first that $(\Phi(v, t))(\mathbf{x})=\Phi(v, t)$, i.e., $\Phi: \mathscr{V} \times \mathrm{I} \rightarrow$ $(\nu, \infty)$. Moreover, if $v_{k} \rightarrow v$ in $\mathscr{V}$, then assume that for all $\epsilon>0$ there exists $N$ such that $\left|\Phi(v, t)-\Phi\left(v_{k}, t\right)\right|<\epsilon$ for all $t \in \mathrm{I}$ if $k>N$. Then, the constraint set analogous to the previous situation is given by

$$
\mathscr{K}(v)=\left\{w \in L^{p}\left(\mathrm{I} ; W_{0}^{1, r}(\Omega)\right):|(\nabla w(t))(\mathbf{x})|_{\mathbb{R}^{l}} \leq \Phi(v, t) \text { a.e. } t \in \mathrm{I}, \mathbf{x} \in \Omega\right\},
$$

and if $v_{k} \rightarrow v$ in $\mathscr{V}$, we have that

$$
\mathscr{K}\left(v_{k}\right) \rightarrow \mathscr{K}(v) \text { in the Mosco sense (see Definition 1.1), as } k \rightarrow \infty .
$$

Indeed, items i and ii from Definition 1.1 hold true:

i. Let $w \in \mathscr{K}(v)$; then the uniform convergence of $\Phi\left(v_{k}, t\right)$ to $\Phi(v, t)$ and the fact that $\Phi(w)(t) \geq \nu>0$ for all $w \in \mathscr{V}$ and $t \in \mathrm{I}$ implies that $w_{k}:=\frac{\Phi\left(v_{k}, t\right)}{\Phi(v, t)} w$ belongs to $\mathscr{K}\left(v_{k}\right)$ and $w_{k} \rightarrow w$ in $L^{p}\left(\mathrm{I} ; W_{0}^{1, r}(\Omega)\right)$ as $k \rightarrow \infty$ (this follows from the fact that $\frac{\Phi\left(v_{k}, t\right)}{\Phi(v, t)}$ converges uniformly to 1$)$.

ii. Let $w_{k} \in \mathscr{K}\left(v_{k}\right)$ and $w_{k} \rightarrow w$ in $L^{p}\left(\mathrm{I} ; W_{0}^{1, r}(\Omega)\right)$ along a subsequence (that we also denote by $\left.\left\{w_{k}\right\}\right)$. Since $\Phi\left(v_{k}, t\right) \rightarrow \Phi(v, t)$ uniformly, we have $\left|\Phi\left(v_{k_{i}}, t\right)-\Phi(v, t)\right|<$ $1 / i$ for some subsubsequence $\left\{v_{k_{i}}\right\}$ and all $t \in \mathrm{I}$. Mazur's lemma implies that there exists a convex combination $\tilde{w}_{i}:=\sum_{n=i}^{N(i)} \lambda(i)_{n} w_{k_{n}}, \lambda(i)_{n} \geq 0$, and $\sum_{n=i}^{N(i)} \lambda(i)_{n}=1$ such that $\tilde{w}_{k} \rightarrow w$ in $L^{p}\left(\mathrm{I} ; W_{0}^{1, r}(\Omega)\right)$. Hence, $\tilde{w}_{k}(t) \rightarrow w(t)$ in $W_{0}^{1, r}(\Omega)$ a.e. $t \in \mathrm{I}$ (along a subsequence), and in turn $\left(\nabla \tilde{w}_{k}(t)\right)(\mathbf{x}) \rightarrow(\nabla w(t))(\mathbf{x})$ a.e. $\mathbf{x} \in \Omega$ (along another subsequence), i.e., since $\left|\left(\nabla \tilde{w}_{i}(t)\right)(\mathbf{x})\right| \leq \Phi(v, t)+\frac{1}{i}$, then $|(\nabla w(t))(\mathbf{x})| \leq \Phi(v, t)$, a.e. $t \in \mathrm{I}, \mathbf{x} \in \Omega$, or $w \in \mathscr{K}(v)$.

In the case where $\mathscr{V}_{n} \equiv \mathscr{V}, \mathscr{H}_{n} \equiv \mathscr{H}$, and $\mathscr{K}_{n}(\cdot) \equiv \mathscr{K}(\cdot)$, conditions A4, A5, and $\mathrm{A} 7$ are fulfilled. We also note that the previous considerations can be extended to more general operators of the form $(\Phi(v, t))(\mathbf{x})=\phi(\mathbf{x}) \Phi_{0}(v, t)$, where $\Phi_{0}$ is of the type described above and $\phi(\mathbf{x}) \in L_{\nu}^{\infty}(\Omega)$. 
When the constraint set is determined by (5.3), compatibility and regularity conditions are difficult to enforce beyond rather simple examples. In fact, suppose that $\Psi$ is a bounded linear functional over $L^{p}(\mathrm{I} ; V)$; then the compatibility and regularity conditions are satisfied if $\Phi(v)=|\Psi(v)|+\nu$, with $\nu>0$. However, if $\psi$ is a bounded linear functional over $V$, then for $\Phi(v, t)=|\psi(v(t))|+\nu$, in general, neither of those conditions are satisfied. Due to the nature of the problem, we are interested in operators $\Phi$ that satisfy the following: If $v \in \mathscr{V} \equiv L^{p}(\mathrm{I} ; V)$, then, for $t \in \mathrm{I}$ fixed, $(\Phi(v, t))(\mathbf{x}) \equiv\left(\Phi\left(v \chi_{(0, t)}, t\right)\right)(\mathbf{x})$, i.e., the expression of $(\Phi(v, t))(\mathbf{x})$ depends only on the values of $v=v(s)$ for $0 \leq s \leq t$ and $t$. Further, we consider

$$
\Phi(v, t)=\theta(t, \Gamma(v, t)), \quad \text { where } \quad \Gamma(v, t)=\int_{0}^{t} k(t-s)(g, v(s))_{V} \mathrm{~d} s,
$$

with $k \in C^{m}(\overline{\mathrm{I}} ; \mathbb{R})$ for $m \geq 1, g \in V^{\prime}$, and $\theta: \mathrm{I} \times \mathbb{R} \rightarrow \mathbb{R}$. In this case, $\Gamma$ : $L^{p}(\mathrm{I} ; V) \rightarrow C(\overline{\mathrm{I}} ; \mathbb{R})$ is a linear bounded operator and a simple application of the Arzelà-Ascoli theorem shows that $\Gamma$ is a compact operator and, hence, it is also completely continuous, i.e., $\Gamma\left(v_{k}, t\right) \rightarrow \Gamma(v, t)$ uniformly in $t \in \overline{\mathrm{I}}$ whenever $v_{k} \rightarrow v$ in $L^{p}(\mathrm{I} ; V)$. Consequently, if $\theta$ is uniformly continuous, then $\theta\left(t, \Gamma\left(v_{k}, t\right)\right) \rightarrow \theta(t, \Gamma(v, t))$ uniformly in $t \in \overline{\mathrm{I}}$. Therefore, in these circumstances, we observe Mosco convergence $\mathscr{K}\left(v_{k}\right) \rightarrow \mathscr{K}(v)$ when $v_{k} \rightarrow v$ in $L^{p}(\mathrm{I} ; V)$ by the first part of this section. A straightforward generalization of this and what follows can be carried out when $g \in L^{p^{\prime}}$ (I; $V^{\prime}$ ) and $(\Phi(v, t))(\mathbf{x})=\theta(t, \Gamma(v, t)) \eta(\mathbf{x})$ with $\eta \in L_{\nu}^{\infty}(\Omega)$.

The compatibility condition is satisfied for certain forms of $k$ and $\theta$. For example, if $\theta(t, \beta)=|\beta|+\nu$ and $k(\alpha)=\alpha^{2} / 2$, then $\int_{0}^{t} k(t-s)(g, v(s))_{V} \mathrm{~d} s=$ $\int_{0}^{t} \int_{0}^{s} \int_{0}^{r}(g, v(q))_{V} \mathrm{~d} q \mathrm{~d} r \mathrm{~d} s$ (by integration by parts). Define $F(s)=\int_{0}^{s} \int_{0}^{r}(g, v(q))_{V} \mathrm{~d} q \mathrm{~d} r$ for a fixed $v \in L^{p}(\mathrm{I} ; V)$, which implies $|F(s)| \leq M_{v}$ for some $M_{v}>0$. Now, if $w \in$ $\mathscr{K}(v)$, then we have that $e^{-a \tau} S(\tau) w$ is bounded by $e^{-a \tau}\left(\int_{0}^{t-\tau} F(s) \mathrm{d} s+\nu\right)($ for $t>\tau)$, and if we assume that $a>M_{v} / \nu$ (which implies that $M_{v} \tau \leq\left(e^{a \tau}-1\right) \nu$ ), then we have $e^{-a \tau}\left(\left|\int_{0}^{t-\tau} F(s) \mathrm{d} s\right|+\nu\right) \leq\left|\int_{0}^{t} F(s) \mathrm{d} s\right|+\nu$ and consequently $e^{-a \tau} S(\tau) w \in \mathscr{K}(v)$. As a result, the compatibility condition holds true.

Since compatibility conditions need only hold in a ball (see the remark at the end of the proof of Theorem 1), the same analysis can be applied (and hence the compatibility condition is satisfied), for example, to $\theta(t, \beta)=\epsilon \beta+\nu$ for some $0<$ $\epsilon \ll 1$. If $t \mapsto \phi(t) \eta(\mathbf{x})$ satisfies the regularity condition from the previous section, it can be proven that for $\theta(t, \beta)=\phi(\epsilon \beta+t)$, we have that $(\Phi(v, t))(\mathbf{x})=\theta(t, \Gamma(v, t)) \eta(\mathbf{x})$ satisfies compatibility and regularity conditions for some small enough $\epsilon>0$ and $k(\alpha)=(\alpha)^{m}$ with $m \geq 2$ (notice that in this case the map $t \mapsto \Gamma(v, t)$ is at least twice differentiable). These same ideas can be generalized to $\Gamma$ operators for which the kernel " $k(t-s)$ " is replaced by " $k(t, s)$ " with $k: \mathrm{I} \times \mathrm{I} \rightarrow \mathbb{R}$ producing sufficiently regular $\Gamma$.

5.4. Nonzero initial conditions. Our study above shows that there exists a $u \in \mathscr{D}(L ; \mathscr{V})$ that solves the problem

$$
\begin{gathered}
\text { Find } u \in \mathscr{K} \cap \mathscr{D}\left(L ; \mathscr{V}^{\prime}\right):\left(u^{\prime}+\mathcal{A}(u)-f, v-u\right)_{\mathscr{V}} \geq 0 \quad \forall v \in \mathscr{K} \text { and } \\
u(0)=0 .
\end{gathered}
$$

Let $u_{0} \in \mathbf{K}(0) \subset V$, where $t \mapsto \mathbf{K}(t)$ is the mapping needed in $\mathscr{K}=\{v \in \mathscr{V}: v(t) \in$ $\mathbf{K}(t)$ a.e. $t \in \mathrm{I}\}$, and let $q_{\eta}(t)=e^{-\eta t^{2}}, \mathcal{A}^{*}(u):=\mathcal{A}\left(u+q_{\eta}(t) u_{0}\right)$, and $f^{*}=f-q_{\eta}^{\prime}(t) u_{0}$. Clearly, if $\mathcal{A}$ satisfies H1, H2, and H3, so does $\mathcal{A}^{*}$ (and vice versa). Similarly, if 
$f \in \mathscr{D}\left(L ; \mathscr{V}^{\prime}\right)$, then the same is true for $f^{*}$ (note that $q_{\eta}^{\prime}(0)=0$ ). Introduce the variables $u^{*}=u-q_{\eta}(t) u_{0}$ and $\mathscr{K}^{*}=\mathscr{K}-q_{\eta}(t) u_{0}$. Then, $u^{*}$ satisfies the problem

Find $u \in \mathscr{K}^{*}:\left(u^{\prime}+\mathcal{A}^{*}(u)-f^{*}, v^{*}-u^{*}\right)_{\mathscr{V}} \geq 0 \quad \forall v \in \mathscr{K}^{*}$ and

$$
u(0)=u_{0}
$$

Clearly, the solution $u^{*}$ depends on the parameter $\eta$, i.e., $u^{*} \equiv u_{\eta}^{*}$ and although taking the limit $\eta \rightarrow \infty$ might be problematic $\left(f^{*}=f-q_{\eta}^{\prime}(t) u_{0}\right.$ becomes singular and the set $\mathscr{K}^{*}=\mathscr{K}-q_{\eta}(t) u_{0}$ would suffer a "discontinuity" at the origin and as a consequence regularity and compatibility conditions might get lost), we can take $\eta$ large enough such that $\mathscr{K}^{*} \simeq \mathscr{K}$, in the sense that if $v \in \mathscr{K}$, then there exists some $v_{\eta} \in \mathscr{K}^{*}$ such that $v_{\eta} \rightarrow v$ as $\eta \rightarrow \infty$.

6. Algorithm. Theorem 1 provides an approximation scheme suitable for implementation. For this purpose we introduce an algorithm based on semidiscretization that falls inside the scope of the aforementioned theorem. Let $\mathscr{V}=L^{p}(\mathrm{I} ; V)$ (with $p \geq 2)$, where $\mathrm{I}=(0, T)$ with $0<T<\infty$ and $\mathscr{V}_{n}=V^{n}:=V \times V \times \cdots \times V(n$ copies of $V)$ with norm $|w|_{\mathscr{V}_{n}}=\left(k \sum_{m=1}^{n}\left|w^{m}\right|_{V}^{p}\right)^{1 / p}, k=\frac{T}{n}$, and where $w=\left\{w^{m}\right\}_{m=1}^{n} \in \mathscr{V}_{n}$. We assume that $\left(V, H, V^{\prime}\right)$ is a Gelfand triple and hence $\left(\mathscr{V}, \mathscr{H}, \mathscr{V}^{\prime}\right)$ and $\left(\mathscr{V}_{n}, \mathscr{H}_{n}, \mathscr{V}_{n}^{\prime}\right)$ are as well, with $\mathscr{H}=L^{2}(\mathrm{I} ; H)$ and $\mathscr{H}_{n}=H^{n}$. Then, consider $P_{n} \in \mathscr{L}\left(\mathscr{V}, \mathscr{V}_{n}\right)$ and $E_{n} \in \mathscr{L}\left(\mathscr{V}_{n}, \mathscr{V}\right)$ defined as

$$
P_{n} v=\left\{\frac{1}{k} \int_{\mathrm{I}_{m}} v(t) \mathrm{d} t\right\}_{m=1}^{n}, \quad\left(E_{n} w\right)(t)=\sum_{m=1}^{n} w^{m} \chi_{\mathrm{I}_{m}}(t),
$$

where $v \in \mathscr{V}, w=\left\{w^{m}\right\}_{m=1}^{n} \in \mathscr{V}_{n}$, and $\mathrm{I}_{m}=((m-1) k, m k)$ for $m=1, \ldots, n$ (we also extend the latter to $m \in \mathbb{Z}$ ).

An application of Hölder's inequality and the definition of the norm in $\mathscr{V}_{n}$ proves $\left|P_{n} v\right|_{\mathscr{V}_{n}} \leq|v|_{\mathscr{V}}$ and also $\left|E_{n} w\right|_{\mathscr{V}}=|w|_{\mathscr{V}_{n}}$. Thus A1 holds true. Assumption A2 holds for step functions and by density of step functions (and because $\left|P_{n}\right|_{\mathscr{L}\left(\mathscr{V}, \mathscr{V}_{n}\right)}$ and $\left|E_{n}\right|_{\mathscr{L}\left(\mathscr{V}_{n}, \mathscr{V}\right)}$ are uniformly bounded), so we can generalize this to all of $\mathscr{V}_{n}$. Finally, A3 is proven just by using the definition of $P_{n}$ and $E_{n}$. Hence, Assumption 1 holds true for our semidiscrete scheme. Also, it is useful to note that from the definition of $P_{n}$ and $E_{n}$, we observe that the restriction of the adjoints $P_{n}^{\prime}\left(\right.$ to $\left.\mathscr{V}_{n}\right)$ and $E_{n}^{\prime}$ (to $\mathscr{V}$ ) are given by $\left.P_{n}^{\prime}\right|_{\mathscr{V}_{n}}=E_{n}$ and $\left.E_{n}^{\prime}\right|_{\mathscr{V}}=P_{n}$.

We will make use of Theorem 1 (and Corollary 3.4) in order to prove convergence of our numerical scheme. For that purpose, we first show that if we have $\left(L_{n}, \mathcal{A}_{n}, f_{n}\right) \stackrel{\boldsymbol{\Delta}}{\longrightarrow}(L, \mathcal{A}, f)$ (Definition 3.3) for some sequences $\left\{L_{n}\right\},\left\{\mathcal{A}_{n}\right\}$, and $\left\{f_{n}\right\}$ and some $(L, \mathcal{A}, f)$, then each of the approximate problems (3.7) reduces to a finite number of well-posed elliptic-type QVI problems equivalent to the ones studied in [19]. Although the spectrum of choices for $\left\{\mathcal{A}_{n}\right\}$ and $\left\{f_{n}\right\}$ is abundant, a useful choice of the sequence $\left\{L_{n}\right\}$ relies heavily on the choice of the extension operators $E_{n}$ and the projection operators $P_{n}$, and the approximation properties of this sequence require careful examination as we observe in Proposition 6.1. For example, given $f \in \mathscr{V} \subset \mathscr{V}^{\prime}$, the sequence $\left\{f_{n}\right\}$ can be determined by $f_{n}=P_{n} f$. Then, by the proof of A2 in the previous paragraph, we infer that $E_{n} P_{n} f \rightarrow f$ as $n \rightarrow \infty$, and as noted above we have $\left.P_{n}^{\prime}\right|_{\mathscr{V}_{n}}=E_{n}$ and $P_{n} f \in \mathscr{V}_{n}$. Therefore, $P_{n}^{\prime} f_{n} \rightarrow f$ in $\mathscr{V}$ (and hence in $\mathscr{V}^{\prime}$ ) for $n \rightarrow \infty$ as required in Definition 3.3.

We assume throughout this section that $\mathcal{A}: \mathscr{V} \rightarrow \mathscr{V}^{\prime}$ is of the form $(\mathcal{A}(w))(t)=$ $A(w(t))$ for $w \in \mathscr{V}$ and $A: V \rightarrow V^{\prime}$ as in Proposition 2.1, and that the sequence 
$\left\{\mathcal{A}_{n}\right\}$ of the form $\mathcal{A}_{n}(v)=\left\{A_{n}\left(v^{m}\right)\right\}_{m=1}^{n}$ for $v=\left\{v^{m}\right\}_{m=1}^{n}$ satisfies the convergence condition iii in Definition 3.3 for some $A_{n}: V \rightarrow V^{\prime}$ with $n \in \mathbb{N}$.

We suppose that the constraint set is given by $\mathscr{V} \ni v \mapsto \mathscr{K}(v) \in 2^{\mathscr{V}}$, thus depending on $v$, such that $\mathscr{K}(v)=\{v \in \mathscr{V}: v(t) \in \mathbf{K}(v, t)$ a.e. $t \in \mathrm{I}\}$, and that $\mathbf{K}$ is determined as in (5.3), i.e.,

$$
\mathbf{K}(v, t):=\left\{y \in V:|(G y)(\mathbf{x})|_{\mathbb{R}^{l}} \leq(\Phi(v, t))(\mathbf{x}) \text { a.e. in } \Omega\right\},
$$

where $G \in \mathscr{L}(V, W)$ is given as in section 5.2. Our approach relies heavily on the assumption that if $v \in \mathscr{V} \equiv L^{p}(\mathrm{I} ; V)$, then, for $t \in \mathrm{I}$ fixed, $(\Phi(v, t))(\mathbf{x}) \equiv$ $\left(\Phi\left(v \chi_{(0, t)}, t\right)\right)(\mathbf{x})$, that is, the expression of $(\Phi(v, t))(\mathbf{x})$ depends only on the values of $v=v(s)$ for $0 \leq s \leq t$.

The time-discrete approximation

$$
\mathscr{K}_{n}(v)=\left\{v \in \mathscr{V}_{n}: v^{1}=0 \quad \text { and } \quad v^{m} \in \mathbf{K}_{n}(v, m) \text { for } m=2, \ldots, n\right\}
$$

is defined by

$$
\mathbf{K}_{n}(v, m):=\left\{y \in V:|(G y)(\mathbf{x})|_{\mathbb{R}^{l}} \leq\left(\Phi_{n}(v, m)\right)(\mathbf{x}) \text { a.e. in } \Omega\right\} .
$$

We assume also that the assumptions A4-A7 are satisfied. For example, consider

$$
\Phi(v, t)=\left|\int_{0}^{t} K(t-s)(g, v(s))_{V} \mathrm{~d} s\right|+\nu,
$$

for $\nu>0$ fixed, with $K \in C^{1}(\overline{\mathrm{I}})$ and $g \in V^{\prime}$ for which the compatibility condition A6 is satisfied. Let

$$
\Phi_{n}\left(\left\{v^{m}\right\}_{m=1}^{n}, m\right):=\left|\sum_{j=1}^{m} K(k m-k j)\left(g, v^{j}\right)_{V} k\right|+\nu .
$$

In this case, if $v_{i} \rightarrow v$ in $\mathscr{V}_{n}=V^{n}$, then we have that $v_{i}^{m} \rightarrow v^{m}$ in $V$ for $m=1,2, \ldots, n$. This implies the uniform convergence of $\Phi_{n}\left(\left\{v_{i}^{m}\right\}_{m=1}^{n}, m\right)$ to $\Phi_{n}\left(\left\{v^{m}\right\}_{m=1}^{n}, m\right)$. Now, the same proof as in section 5.3 shows that $\mathscr{K}_{n}\left(v_{i}\right) \rightarrow \mathscr{K}_{n}(v)$ in the Mosco sense as $i \rightarrow$ $\infty$, i.e., assumption A4 is satisfied. To prove A5 we simply observe that if $E_{n} v_{n} \rightarrow v$ in $\mathscr{V}$, then $\Phi\left(E_{n} v_{n}, t\right) \rightarrow \Phi(v, t)$ and $\Phi_{n}\left(v_{n}, m\right) \rightarrow \Phi(v, t)$ (actually $E_{n}\left(\Phi_{n}\left(v_{n}, m\right)\right) \rightarrow$ $\Phi(v, t))$, both uniformly, and hence, if $v_{n} \in \mathscr{K}_{n}\left(v_{n}\right)$, then an analogous proof to the one in b of section 5.3 shows the desired result. Finally, A7 follows from using similar ideas as in the previous statement and the proof of section 5.3.

For our further analysis we make use of the semigroup of translations $S(\tau)$ as defined in (5.1), with generator $-L=-\frac{\mathrm{d}}{\mathrm{d} t}$ and with domain $\mathscr{D}\left(L ; \mathscr{V}^{\prime}\right)$ characterized as in (5.2). From now on we assume that if $v \in \mathscr{D}\left(L ; \mathscr{V}^{\prime}\right) \cap \mathscr{V}$, then (for the sake of simplicity) its derivative $v^{\prime}$ is defined to be zero outside the interval $(0, T)$. Note that this extension does not change the absolute continuity of $v$. Hence $v(t)=0$ for $t<0$, since $v(0)=0$ and $v(t)=\int_{0}^{T} v^{\prime}(s) \mathrm{d} s$ for $t \geq T$. We now prove the crucial result needed for the convergence of $\left\{L_{n}\right\}$ to $L$ in the sense of Definition 3.3.

Proposition 6.1. Let $L_{n}=\frac{I-F(1 / n)}{1 / n}$ (the structure required in (3.1)), where $F(1 / n) w=\left\{0, w_{1}, w_{2}, \ldots, w_{n-1}\right\} \in \mathscr{V}_{n}$ for $w=\left\{w_{m}\right\}_{m=1}^{n} \in \mathscr{V}_{n}$. Then, for $v \in$ $\mathscr{D}\left(L ; \mathscr{V}^{\prime}\right) \cap \mathscr{V}, P_{n}^{\prime} L_{n} P_{n} v$ is given by

$$
\left(P_{n}^{\prime} L_{n} P_{n} v\right)(t)=\sum_{j=1}^{n} \frac{1}{k^{2}}\left(\int_{\mathrm{I}_{j}} v(s) \mathrm{d} s-\int_{\mathrm{I}_{j-1}} v(s) \mathrm{d} s\right) \chi_{\mathrm{I}_{j}}(t),
$$

Copyright $@$ by SIAM. Unauthorized reproduction of this article is prohibited. 
and it satisfies

$$
\lim _{n \rightarrow \infty} P_{n}^{\prime} L_{n} P_{n} v=v^{\prime} \text { in } \mathscr{V}^{\prime}
$$

Proof. If $v \in \mathscr{D}\left(L ; \mathscr{V}^{\prime}\right)$, then it is absolutely continuous, $v(0)=0$, and it admits the representation $v(t)=\int_{0}^{t} v^{\prime}(t) \mathrm{d} t$ with $v^{\prime} \in \mathscr{V}^{\prime}$ (and $v^{\prime}(t)=0$ if $t \notin(0, T)$ ). Using this, by a change of variables, $P_{n}^{\prime} L_{n} P_{n} v$ is given by

$$
\left(P_{n}^{\prime} L_{n} P_{n} v\right)(t)=\sum_{j=1}^{n} \frac{1}{k^{2}} \int_{\mathrm{I}_{j-1}}\left(\int_{s}^{s+k} v^{\prime}(r) \mathrm{d} r\right) \mathrm{d} s \chi_{\mathrm{I}_{j}}(t)
$$

Then, by elementary properties of the Bochner integral and Hölder's inequality, we observe

$$
\begin{aligned}
\left|P_{n}^{\prime} L_{n} P_{n} v-v^{\prime}\right|_{L^{p^{\prime}\left(I ; V^{\prime}\right)}}^{p^{\prime}} & =\sum_{j=1}^{n} \int_{\mathrm{I}_{j}}\left|\frac{1}{k^{2}} \int_{\mathrm{I}_{j-1}}\left(\int_{s}^{s+k} v^{\prime}(r) \mathrm{d} r\right) \mathrm{d} s-v^{\prime}(t)\right|_{V^{\prime}}^{p^{\prime}} \\
& \leq \sum_{j=1}^{n} \int_{\mathrm{I}_{j}}\left(\frac{1}{k} \int_{\mathrm{I}_{j-1}}\left(\frac{1}{k} \int_{s}^{s+k}\left|v^{\prime}(r)-v^{\prime}(t)\right|_{V^{\prime}} \mathrm{d} r\right) \mathrm{d} s\right)^{p^{\prime}} \\
& \leq \sum_{j=1}^{n} \int_{\mathrm{I}_{j}} \frac{1}{k} \int_{\mathrm{I}_{j-1}}\left(\frac{1}{k} \int_{s}^{s+k}\left|v^{\prime}(r)-v^{\prime}(t)\right|_{V^{\prime}} \mathrm{d} r\right)^{p^{\prime}} \mathrm{d} s .
\end{aligned}
$$

Now, let $M$ denote the Hardy-Littlewood maximal function; that is, $(M f)(x):=$ $\sup _{Q \ni x} \frac{1}{|Q|} \int_{Q}|f(r)| \mathrm{d} r$ when $f \in L^{1}(\mathbb{R})$. It is a well-known fact that if $f \in L^{p}(\mathbb{R})$ for $p>1$, then $M f \in L^{p}(\mathbb{R})$, and even more $|M f|_{L^{p}(\mathbb{R})} \leq C_{p}|f|_{L^{p}(\mathbb{R})}$ for some $C_{p}$ (not depending on $f$ ). Moreover, the previous inequality is strengthened by the FeffermanStein inequality (see Theorem 2.12 in [15] or [13]), which guarantees the existence of a constant $C$ (depending only on $1<p<\infty$ ) such that for any measurable functions $f, w: \mathbb{R}^{n} \rightarrow \mathbb{R}$ and with $w$ nonnegative, we have

$$
\int_{\mathbb{R}}|(M f)(s)|^{p} w(s) \mathrm{d} s \leq C \int_{\mathbb{R}}|f(s)|^{p}(M w)(s) \mathrm{d} s .
$$

Hence, for a fixed $t \in \mathrm{I}$, let $f(s):=\left|v^{\prime}(s)-v^{\prime}(t)\right| \chi_{\left(\mathrm{I}_{j-1} \cup \mathrm{I}_{j}\right)}(s)$ and $w(s):=\chi_{\mathrm{I}_{j-1}}(s)$; then

$$
\int_{\mathrm{I}_{j-1}}\left(\frac{1}{k} \int_{s}^{s+k}\left|v^{\prime}(r)-v^{\prime}(t)\right|_{V^{\prime}} \mathrm{d} r\right)^{p^{\prime}} \mathrm{d} s \leq C \int_{\mathrm{I}_{j-1} \cup \mathrm{I}_{j}}\left|v^{\prime}(s)-v^{\prime}(t)\right|^{p^{\prime}} \mathrm{d} s
$$

where we have used $|(M w)(s)| \leq 1$. We also note that $C>0$ depends only on $1<$ $p^{\prime}<\infty$ and does not depend on $t \in \mathrm{I}$ nor $v$. Hence, recalling that $\mathrm{I}_{j}=(k(j-1), k j)$, 
by a change of variables and Fubini's theorem, we have

$$
\begin{aligned}
\left|P_{n}^{\prime} L_{n} P_{n} v-v^{\prime}\right|_{L^{p^{\prime}\left(I ; V^{\prime}\right)}}^{p^{\prime}} & \leq C \sum_{j=1}^{n} \int_{t_{j-1}}^{t_{j}} \frac{1}{k} \int_{t_{j-2}}^{t_{j}}\left|v^{\prime}(s)-v^{\prime}(t)\right|_{V^{\prime}}^{p^{\prime}} \mathrm{d} s \mathrm{~d} t \\
& \leq C \sum_{j=1}^{n} \int_{t_{j-1}}^{t_{j}} \frac{1}{k} \int_{t-2 k}^{t+k}\left|v^{\prime}(s)-v^{\prime}(t)\right|_{V^{\prime}}^{p^{\prime}} \mathrm{d} s \mathrm{~d} t \\
& =C \int_{0}^{T} \frac{1}{k} \int_{-2 k}^{k}\left|v^{\prime}(t+\sigma)-v^{\prime}(t)\right|_{V^{\prime}}^{p^{\prime}} \mathrm{d} \sigma \mathrm{d} t \\
& =C \frac{1}{k} \int_{-2 k}^{k} \int_{0}^{T}\left|v^{\prime}(t+\sigma)-v^{\prime}(t)\right|_{V^{\prime}}^{p^{\prime}} \mathrm{d} t \mathrm{~d} \sigma \\
& \leq 3 C \sup _{|\sigma| \leq 2 k} \int_{0}^{T}\left|v^{\prime}(t+\sigma)-v^{\prime}(t)\right|_{V^{\prime}}^{p^{\prime}} \mathrm{d} t .
\end{aligned}
$$

Since we have assumed that $v^{\prime} \in L^{p^{\prime}}\left((0, T) ; V^{\prime}\right)$ is zero outside $(0, T)$, it also belongs to $L^{p^{\prime}}\left((-2 k, T+2 k) ; V^{\prime}\right)$. Therefore, the last expression in the previous inequality is $3 C\left(\omega\left(v^{\prime} ; 2 k\right)\right)^{p^{\prime}}$, where $\omega\left(v^{\prime} ; k\right)$ refers to the modulus of continuity (in $L^{p^{\prime}}((-2 k, T+$ $\left.2 k) ; V^{\prime}\right)$ ), which tends to zero as $k \downarrow 0$.

The approximating problems in (3.7) require us to find $u_{n}=\left\{u^{m}\right\}_{m=1}^{n} \in \mathscr{V}_{n}$ (that is, each $u_{m}$ is in $V$ ) that solves

$$
\begin{aligned}
& u^{m} \in \mathbf{K}_{n}\left(u_{n}, m\right) \\
\left(\mathrm{N}_{\mathrm{QVI}}\right) \quad & :\left(\frac{u^{m}-u^{m-1}}{k}+A_{n}\left(u^{m}\right)-f^{m}, v^{m}-u^{m}\right)_{V} \geq 0 \quad \forall v^{m} \in \mathbf{K}_{n}\left(u_{n}, m\right) ; \\
& u^{1}=0
\end{aligned}
$$

for $m=2, \ldots, n$, where $v=\left\{v^{m}\right\}_{m=1}^{n}$.

Since $u^{1}$ is given, and the constraint set $\mathbf{K}_{n}\left(u_{n}, m\right)$ for each $m=2,3, \ldots, n$ does not involve $u^{m+1}, u^{m+2}, \ldots, u^{n}$, finding $u^{m}$ reduces to the resolution of an elliptic QVI. In fact, let $n \in \mathbb{N}$ and $m=2,3, \ldots, n$ be fixed, and given $u^{1}, u^{2}, \ldots, u^{m-1}$, then $u^{m}$ is a fixed point of the mapping $V \ni w \mapsto S_{m}(B, g, \mathbf{K}(w))$, where $u=S_{m}(B, g, \mathbf{K}(w))$ is the unique solution to

$$
\text { Find } u \in \mathbf{K}(w):(B(u)-g, v-u)_{V} \geq 0 \quad \forall v \in \mathbf{K}(w),
$$

where $\mathbf{K}(w) \equiv \mathbf{K}_{n}(w, m):=\mathbf{K}_{n}\left(\left\{u^{1}, u^{2}, \ldots, u^{m-1}, w, 0, \ldots\right\}, m\right), B(u) \equiv B_{n}(u):=$ $A_{n}(u)+u / k$, and $g \equiv g_{n}^{m}=f^{m}-u^{m-1} / k$. We observe that $B: V \rightarrow V^{\prime}$ is strongly monotone, continuous on finite dimensional spaces, and coercive. Moreover, if $v_{j} \rightarrow v$ in $V$, then $\mathbf{K}\left(v_{j}\right) \rightarrow \mathbf{K}(v)$ in the Mosco sense, and hence the solution mapping $w \mapsto S(B, g, \mathbf{K}(w)) \equiv S\left(B_{n}, g_{n}^{m}, \mathbf{K}_{n}(w, m)\right)$ of this elliptic-type QVI is continuous and compact (and there is also some ball invariant to this mapping). Hence, Schauder's fixed point theorem guarantees the existence of a solution to $u=S_{m}(B, g, \mathbf{K}(u))$. However, the well posedness of the elliptic problem (in the sense of the existence of a unique solution) is not obvious. In [19], it was proven that under certain hypotheses on the regularity and norm of the forcing term, the uniform monotonicity of $B$ and the properties of the upper bound mapping for the gradient, the elliptic QVI has a unique solution. Moreover, in the case of linear mappings $A_{n}: V \rightarrow V$, scalability gives uniqueness under even weaker conditions. The uniqueness of solutions of $u=S_{m}(B, g, \mathbf{K}(u))$ is determined by the contraction property of the map 
$w \mapsto S_{m}(B, g, \mathbf{K}(w))$ and the $S_{m}(B, g, \mathbf{K}(\cdot))$-invariance of a ball of a certain positive radius. This implies not only the existence of a unique fixed point of the solution mapping but also that the iteration $w^{\ell+1}=S_{m}\left(B, g, \mathbf{K}\left(w^{\ell}\right)\right)$ with $w^{0}$ chosen small enough converges to this fixed point (for the proof of the contraction property and general conditions by which the latter iteration converges, at least along a subsequence, to a solution of the elliptic-type QVI, see [19]). Hence, under these circumstances, the resolution of each step in $\mathrm{N}_{\mathrm{QVI}}$ is well posed and by a direct application of Theorem 1 we have the following result.

Corollary 6.2. Let $n \in \mathbb{N}, n>1$. Suppose that the problems $\left(\mathrm{N}_{\mathrm{QVI}}\right)$ are well posed (in the sense described above) for all $m=2, \ldots, n$. Let $u_{n}=\left\{u^{m}\right\}_{m=1}^{n}$ be the sequence obtained from $\left(\mathrm{N}_{\mathrm{QVI}}\right)$. Then, $E_{n} u_{n} \rightarrow u$ along a subsequence, with $u$ the solution to $\left(\mathrm{P}_{\mathrm{QVI}}\right)$.

This result, together with the facts discussed in the previous paragraph, determine Algorithm 1 below. The term "Suitable Convergence Criteria" refers to a stopping criterion associated to the search of an approximate solution of $u=S\left(B_{n}, g_{n}^{m}, \mathbf{K}_{n}(u, m)\right)$. In our case, we use a criterion based on the linear convergence of the approximate sequence defined in step 5 of Algorithm 1. This approach follows the one in [19].

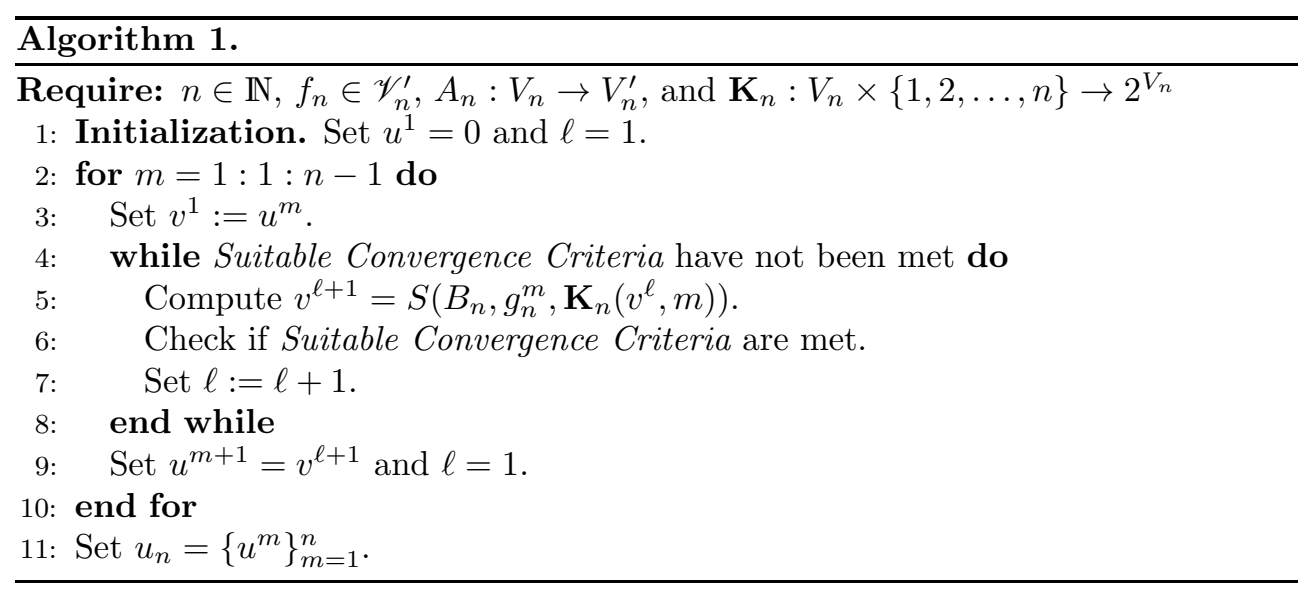

7. Numerics. We consider the approximation of the problem $\left(\mathrm{P}_{\mathrm{QVI}}\right)$ associated with the time realization of the $p$-Laplacian operator (for $p=2$ and $p=3$ ) by $\left(\mathrm{N}_{\mathrm{QVI}}\right)$ and always use $\mathrm{I}:=(0,1)$ and $\Omega:=(0,1) \times(0,1)$. The state space $\mathscr{V}$ is given by $L^{p}(\mathrm{I} ; V)$ with $V:=W_{0}^{1, p}(\Omega)$. It should be noted that the $p$-Laplacian is a potential operator, i.e., the functional $W_{0}^{1, p}(\Omega) \ni u \mapsto \frac{1}{p} \int_{\Omega}|\nabla u|^{p}$ is Gâteaux differentiable and its derivative is given by the $p$-Laplacian. This also implies that an elliptic VI, with the $p$-Laplacian as the associated monotone operator, is equivalent to a constrained optimization problem; this fact is later exploited in the numerical implementation. In this case the Gelfand triple structure associated to the problem is given by $\left(L^{p}(\mathrm{I} ; V), L^{2}\left(\mathrm{I} ; H_{0}^{1}(\Omega)\right), L^{p^{\prime}}\left(\mathrm{I} ; V^{\prime}\right)\right)$, where $V^{\prime}=W^{-1, p^{\prime}}(\Omega)$ is the topological dual of $V$.

Our numerical tests focus on a class of QVIs falling into our general framework. We are, in particular, interested in studying the solution dynamics and the time dependence of the associated active sets. 
The numerical tests are based on the discretization of the approximating problems $\left(\mathrm{N}_{\mathrm{QVI}}\right)$ : Find $u_{n}=\left\{u^{m}\right\}_{m=1}^{n}$, with $u^{m} \in W_{0}^{1, p}(\Omega)$ for $m=1,2, \ldots, n$, that solves

$$
\begin{aligned}
& u^{m} \in \mathbf{K}_{n}\left(u_{n}, m\right) \\
& :\left(\frac{u^{m}-u^{m-1}}{k}-\delta \Delta_{p}\left(u^{m}\right)-f^{m}, v^{m}-u^{m}\right)_{V} \geq 0 \quad \forall v^{m} \in \mathbf{K}_{n}\left(u_{n}, m\right), \\
& u^{1}=0,
\end{aligned}
$$

for $m=2, \ldots, n$, where $v=\left\{v^{m}\right\}_{m=1}^{n}, \delta>0$ is a fixed parameter, and

$$
\mathbf{K}_{n}(v, m):=\left\{y \in V:|(\nabla y)(\mathbf{x})|_{\mathbb{R}^{l}} \leq\left(\Phi_{n}(v, m)\right)(\mathbf{x}) \text { a.e. in } \Omega\right\} .
$$

The discretization scheme in time is determined by $\left(\mathrm{N}_{\mathrm{QVI}}\right)$; that is, $k=\frac{T}{n}$ is the mesh size of the uniform mesh on $\mathrm{I}=(0, T)=(0,1)$. Our finite difference approximation scheme in space has $M^{2}$ uniformly distributed nodes implying the mesh size $h=$ $1 /(M+1)$ in each coordinate direction. At a node $\mathbf{x}_{i j}=\left(x_{i}, x_{j}\right)$, with $x_{i}=i h$ and $x_{j}=j h$ for $1 \leq i, j \leq M$, we approximate $w\left(\mathbf{x}_{i j}\right)$, for $w \in V$, by $w_{i j}=w\left(x_{i}, x_{j}\right)$ and denote by $w^{h}$ the corresponding discrete approximation of $w$ on the given mesh. We approximate the $V$-norm by $\left|w^{h}\right|_{V}^{p}:=\sum_{i, j=1}^{M}\left|\left(D_{-} w^{h}\right)\right|_{i j}^{p} h^{2}$ with $\left(D_{-} w^{h}\right)_{i j}=\frac{1}{h}\left(w_{i j}-\right.$ $\left.w_{(i-1) j}, w_{i j}-w_{i(j-1)}\right)^{\top}$ and $\left|\left(u^{h}, v^{h}\right)^{\top}\right|_{i j}^{2}=u_{i j}^{2}+v_{i j}^{2}$. The discretization of the second order elliptic operator $-\Delta_{p}: W_{0}^{1, p}(\Omega) \rightarrow W^{-1, p^{\prime}}(\Omega)$ is the one given in [19] and it is also made explicit in section 7.1 below. Also, for fixed $t \in \mathrm{I}$, whenever the pointwise evaluation of $f(t)$ in $\Omega$ is well defined, then $\left(f(t)^{h}, w^{h}\right):=\sum_{i, j=1}^{M} f(t)_{i j} w_{i j} h^{2}$. This is the case when $f(t) \in W_{0}^{1, p}(\Omega) \subset W^{-1, p^{\prime}}(\Omega)$, for instance. In all our examples, $f(t)=g(t) \varphi$, where $g \in C^{1}(\overline{\mathrm{I}})$ with $g(0)=1$ and $\varphi \in W_{0}^{1, p}(\Omega)$; specifically, the forcing term $f(t)=f(t, x, y)$ is parametrized by $r_{1}, r_{2}, r_{3}$, and $r_{4}$ as

$$
f(t, x, y)=r_{1} \chi_{\left(r_{4}, T\right)}(t)\left(1-e^{-r_{3}\left(t-r_{4}\right)^{r_{2}}}\right) \varphi(x, y),
$$

with $\varphi(x, y)=(x y(x-1)(y-1))^{2}$, where $r_{1}>0, r_{2} \geq 2$. This warrants that $\left.f \in \mathscr{D}\left(L^{2} ; \mathscr{V}\right)\right), r_{3}>0$, and $0<r_{4}<T$. The map $t \mapsto f(t)$ is monotonically increasing, i.e., $f\left(t_{1}, x, y\right) \leq f\left(t_{2}, x, y\right)$ for $t_{1} \leq t_{2}$, and we observe $\lim _{t \rightarrow \infty} f(t)=r_{1} \varphi$. Thus, it seems possible to obtain certain limiting behavior, in time, of the solution to the QVI (for some classes of upper bound operators $\Phi$ ).

For fixed $1 \leq m \leq n-1$, given the sequence $\left\{v^{\ell}\right\}$ generated as in step 5 of Algorithm 1, we define the linear convergence coefficient sequence $\left\{\mu^{\ell}\right\}$ by $\mu^{\ell}:=$ $\left|v^{\ell+2}-v^{\ell+1}\right|_{V} /\left|v^{\ell+1}-v^{\ell}\right|_{V}$. The convergence criteria of step 6 in Algorithm 1 are declared as met as soon as for some $\ell>\ell_{0}$,

( $\left.\mathrm{SP}_{\text {convergence }}\right)$

$$
\left.\begin{array}{l}
\max _{\ell-\ell_{0} \leq r, s \leq \ell}\left|\mu^{r}-\mu^{s}\right|<\epsilon_{1}, \\
\frac{\left|v^{2}-v^{1}\right|_{V}}{1-\mu^{\ell}} \prod_{j=1}^{\ell} \mu^{j}<\epsilon_{2},
\end{array}\right\}
$$

with some prescribed $\ell_{0} \in \mathbb{N}, \epsilon_{1}>0$, and $\epsilon_{2}>0$. Then, Algorithm 1 is stopped. In our numerical tests for examples 2 and 3 , using $\ell_{0}=4, \epsilon_{1}=1 \mathrm{e}-3$, and $\epsilon_{2}=1 \mathrm{e}-4$, the conditions in $\left(\mathrm{SP}_{\text {convergence }}\right)$ are satisfied for $6 \leq \ell \leq 12$. For a detailed explanation of these convergence criteria, see [19].

7.1. Step 5 of Algorithm 1. The computation in step 5 of Algorithm 1 is based on a penalty-method combined with a semismooth Newton iteration. This approach 
has been successfully applied in [19] and a very brief summary is included here for the sake of completeness and to keep this section self-contained. Let $u$ be the unique solution to the problem

$$
\text { Find } u \in \mathbf{K}:(B(u)-g, v-u)_{V} \geq 0 \quad \forall v \in \mathbf{K},
$$

where $\mathbf{K}=\{v \in V:|\nabla v(\mathbf{x})| \leq \varphi(\mathbf{x})$ a.e. in $\Omega\}, \varphi \in L_{\nu}^{\infty}(\Omega)$, and $B(v)=-\Delta_{p}(v)+\frac{v}{k}$ for some $k>0$ and $g \in V^{\prime}$. Then, by classical optimization results, $u \in V$ is also a solution to

$$
\left.\begin{array}{l}
\min J(u):=-\frac{1}{p}\left(\Delta_{p}(u), u\right)_{V}+\frac{1}{2 k}(u, u)_{V}-(g, u)_{V} \text { over } u \in V \\
\text { subject to } u \in \mathbf{K}
\end{array}\right\} .
$$

The penalized problem associated with $(\mathrm{P})$ is given by

$$
\min J_{\gamma}(u):=J(u)+\frac{\gamma}{2}\left|(|\nabla u|-\varphi)^{+}\right|_{L^{2}(\Omega)}^{2} \text { over } u \in V,
$$

where $\gamma \geq 0$ is the penalty parameter and $(\cdot)^{+}$denotes $\max (0, \cdot)$ in the pointwise sense. For each $\gamma \geq 0$, let $u_{\gamma}$ be a solution to $\left(\mathrm{P}_{\gamma}\right)$ (its existence is shown in [19]), and let $\left\{\gamma_{n}\right\}$ be a nonnegative sequence tending to infinity. Then $u_{\gamma_{j}} \rightarrow u$ in $V$, along a subsequence, where $u \in V$ is a solution to (P) (see [19]). The choice $\left\{\gamma_{n}\right\}$ is delicate (especially in the case of $p>2$ due to the nonlinearity of $-\Delta_{p}$, but also when $\phi(\mathbf{x})$ is not constant) and requires a continuation technique that is fully described in [19].

First order optimality conditions for $\left(\mathrm{P}_{\gamma}\right)$ are determined by $F_{\gamma}(u)=0$, where

$$
F_{\gamma}(u)=-\Delta_{p}(u)+\frac{u}{k}-g+\gamma \mathfrak{P}(u),
$$

with $\mathfrak{P}(u)=\nabla^{*}(|\nabla u|-\varphi)^{+} q(\nabla u) \nabla$, where $q(v)(\mathbf{x})=\frac{v(\mathbf{x})}{|v(\mathbf{x})|}$ if $|v(\mathbf{x})|>0$ and $q(v)(\mathbf{x}) \in$ $\bar{B}_{1}(0)^{N}$ otherwise. Then, $F_{\gamma}$ is Newton (or slantly) differentiable (see $[9,18]$ ), i.e., semismooth, as a mapping from $W_{0}^{1, p}(\Omega)$ to $W^{-1, s}(\Omega)$ for some $s$ restricted by $p$. This suggests that a semismooth Newton method can be used to obtain a solution to $F_{\gamma}(u)=0$ in function space; for a full discussion on advantages and restrictions for the method, see [19]. Otherwise if $s$ does not satisfy such restrictions, the method still has a locally superlinear convergence behavior in finite dimensions, but this convergence behavior possibly depends on the mesh size of the discretization.

The discretization of the mapping $F_{\gamma}$ defined in (7.4) is done using the following discretization scheme for the second order elliptic operator $u \mapsto B(a, u):=$ $\nabla^{*}(a(x, y) \nabla u)$ with $a(x, y)>0$ in $\Omega$. For a uniform mesh of size $h>0$, the discretization of $B$ is determined by $[B]^{h}$ defined as

$$
\begin{aligned}
-[B]^{h}\left(a^{h}, u^{h}\right)_{i j} & =\left(\alpha_{i j}+\alpha_{(i+1) j}\right)\left(u_{(i+1) j}-u_{i j}\right)-\left(\alpha_{i j}+\alpha_{(i-1) j}\right)\left(u_{i j}-u_{(i-1) j}\right) \\
& +\left(\alpha_{i j}+\alpha_{i(j+1)}\right)\left(u_{i(j+1)}-u_{i j}\right)-\left(\alpha_{i j}+\alpha_{i(j-1)}\right)\left(u_{i j}-u_{i(j-1)}\right),
\end{aligned}
$$

with $\alpha_{i j}=a\left(x_{i}, x_{j}\right) /\left(2 h^{2}\right), 1 \leq i, j \leq M$. This scheme is second order accurate and the linear map $u^{h} \mapsto B\left(a^{h}, u^{h}\right)$ is symmetric and positive definite (see [21]).

Let $u_{\gamma}^{h}$ be the solution to $\left[F_{\gamma}\right]^{h}(u)=0$, the discretized version of $F_{\gamma}(u)=0$. Then, under appropriate conditions on $u_{\gamma}^{h}$ (for a detailed explanation, see [19]), we have that $\left[F_{\gamma}\right]^{h}$ is semismooth at $u_{\gamma}^{h}$ (see [33]) and that the Newton iteration associated to the resolution of $\left[F_{\gamma}\right]^{h}(u)=0$ converges superlinearly provided that the iteration is started sufficiently close to $u_{\gamma}^{h}$ (see [33]). In our examples, we stop the Newton iteration when 
the norm of the distance between two successive iterates is below NewtonTol $=1 \mathrm{e}-5$ and the Newton iteration associated to $\left[F_{\gamma_{i}}\right]^{h}(u)=0$ is started using $u_{\gamma_{i-1}}^{h}$ (i.e., the solution to $\left.\left[F_{\gamma_{i-1}}\right]^{h}(u)=0\right)$.

In our examples, for $\gamma \geq 500$ the map $\gamma \mapsto u_{\gamma}$ does not change significantly. When $\gamma=500$, conditions that guarantee feasibility in the $L^{2}(\Omega)$ sense, which is measured by the value of $\frac{\gamma}{2}\left|\left(\left|\nabla u_{\gamma}\right|-\varphi\right)^{+}\right|_{L^{2}(\Omega)}^{2}$, a small variation with respect to the previous iterate, i.e., the value of $\left|u_{\gamma_{i}}-u_{\gamma_{i-1}}\right|_{V}$, and a negligible variation in the subsequent iterates are met; for a detailed explanation of these criteria, see [19]. Hence, it is enough to consider $\gamma_{\text {Final }}=1000$, which is the upper bound of the $\gamma$-sequence generated by our algorithm. Henceforth, the resolution of step 5 of Algorithm 1 (i.e., the computation of $\left.v^{\ell+1}=S\left(B_{n}, g_{n}^{m}, K_{n}\left(v^{\ell}, m\right)\right)\right)$ is determined as $v^{\ell+1}=u_{\gamma_{\text {Final }}}$ for the associated penalized problem.

7.2. Example 1. We consider $p=3, \delta=1$. The computational domain consists of $M^{2}$ uniformly distributed nodes in $\Omega=(0,1) \times(0,1)$, where $M=128$ and the mesh size is $h=1 /(\mathrm{M}+1)$. The time interval $\mathrm{I}=(0,1)$ is discretized uniformly with mesh size $k=1 / 100$. The parameters for the forcing term are $r_{1}=0.1, r_{2}=2$, $r_{3}=10$, and $r_{4}=0$ and the upper bound operator is taken to be $\Phi=0.03$.

The forcing term $t \mapsto f(t, \mathbf{x})$ at $t=0.02,0.39,1$ is shown in Figures 1(a), 1(b), and 1 (c), respectively; the approximate solution to the parabolic QVI $t \mapsto u(t, \mathbf{x})$ at the same time instances is given in Figures 1(d), 1(e), and 1(f). Also, the approximate behavior of $t \mapsto|\nabla u(t, \mathbf{x})|-\Phi(t, \mathbf{x})$ at the time steps $t=0.02,0.39,1$ is shown in Figures $1(\mathrm{~g}), 1(\mathrm{~h})$, and $1(\mathrm{i})$. Finally, the approximation to the active set $t \mapsto \mathbb{A}(t)=$ $\{\mathbf{x} \in \Omega:|\nabla u(t, \mathbf{x})|-\Phi(t, \mathbf{x})=0\}$ at the time steps $t=0.39,1$ is observed in Figures $1(\mathrm{j})$ and $1(\mathrm{k})$, respectively.

The limiting behavior of the approximate solution to the QVI $\left(\lim _{t \rightarrow \infty} u(t)=u^{\infty}\right)$ seems to satisfy $\left(-\Delta_{p}\left(u^{\infty}\right)-f^{\infty}, v-u^{\infty}\right)_{V} \geq 0$ for all $v \in K(\Phi)$, where $f^{\infty}=$ $\lim _{t \rightarrow \infty} f(t)$. We refer to [19] for further aspects of this similarity between the timedependent and stationary cases.

7.3. Example 2. We consider $p=2, \delta=0.01$. As above, the computational domain consists of $M^{2}$ uniformly distributed nodes on $\Omega=(0,1) \times(0,1)$, where $M=128$ and the mesh size is $h=1 /(\mathrm{M}+1)$. The time interval $\mathrm{I}=(0,1)$ is discretized into a uniform mesh with mesh size $k=1 / 100$. The parameters for the forcing term are $r_{1}=0.001, r_{2}=2, r_{3}=10^{4}$, and $r_{4}=0$ and the upper bound operator taken to be

$$
\Phi(v, t)=100\left|\int_{0}^{t}\left(\int_{\Omega} v(s, \mathbf{x}) \mathrm{d} \mathbf{x}\right) \mathrm{d} s\right|+0.001 .
$$

The forcing term $t \mapsto f(t, \mathbf{x})$ at $t=0.02,0.10,0.15$ is shown in Figures 2(a), 2(b), and 2(c), respectively; the approximate solution to the parabolic QVI $t \mapsto u(t, \mathbf{x})$ at the same time steps is given in Figures 2(d), 2(e), and 2(f). Also, the approximate behavior of $t \mapsto|\nabla u(t, \mathbf{x})|-\Phi(t, \mathbf{x})$ at the time steps $t=0.02,0.10,0.15$ is shown in Figures 2(g), 2(h), and 2(i). Finally, the approximation to the active set $t \mapsto \mathbb{A}(t)=$ $\{\mathbf{x} \in \Omega:|\nabla u(t, \mathbf{x})|-\Phi(t, \mathbf{x})=0\}$ at the time steps $t=0.10,0.15$ is observed in Figures $2(\mathrm{j})$ and $2(\mathrm{k})$, respectively.

In this example, for $t \gtrsim 1.8$, the approximate solution achieves a steady state and no significant variation is observed subsequently. This is simply explained by the rapid convergence of the forcing term and continuous growth of $t \mapsto \Phi(u, t)$ when $u$ is the approximate solution. In fact, once the active set is empty, no significant variation 


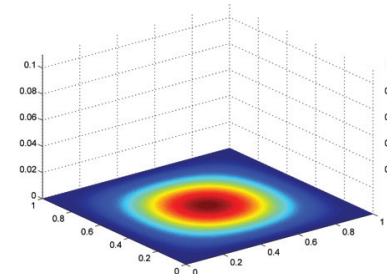

(a)

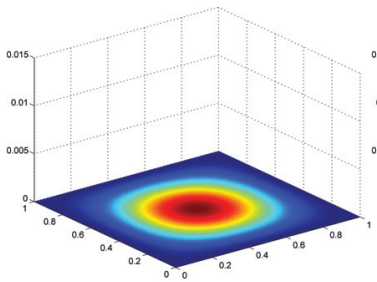

(d)

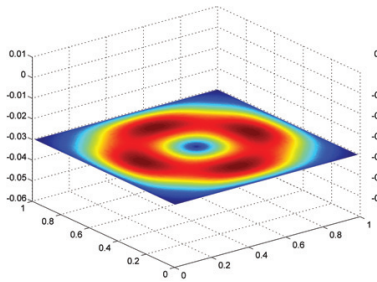

$(\mathrm{g})$

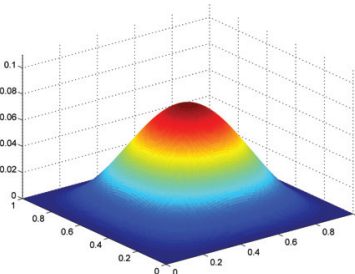

(b)

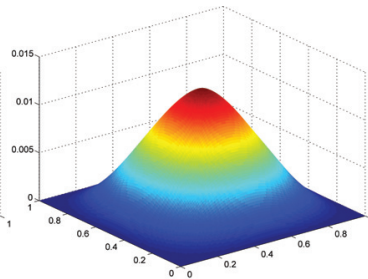

(e)

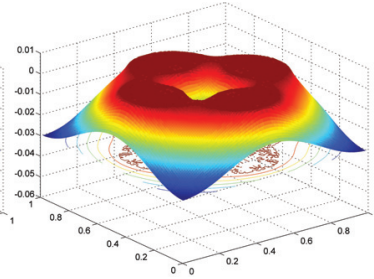

(h)

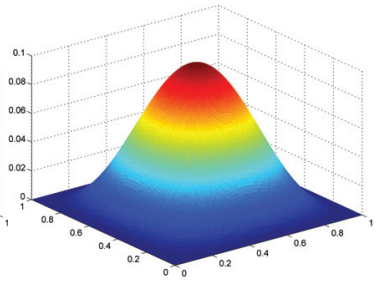

(c)

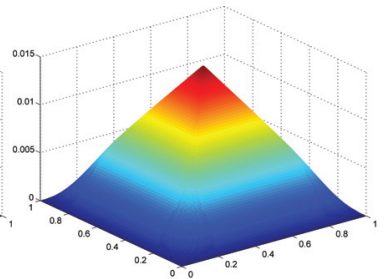

(f)

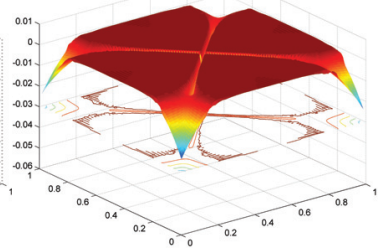

(i)

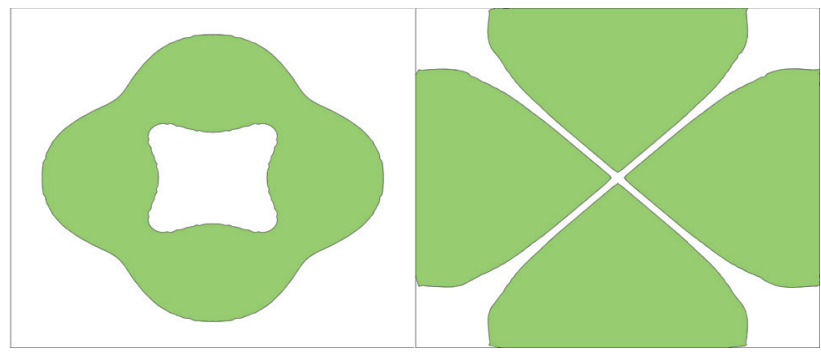

$(\mathrm{j})$

$(\mathrm{k})$

FIG. 1. The forcing term $t \mapsto f(t, \mathbf{x})$ at $t=0.02,0.39,1$ is shown in Figures $1(\mathrm{a}), 1(\mathrm{~b})$, and $1(\mathrm{c})$, respectively. $(t, \mathbf{x}) \mapsto u(t, \mathbf{x})$ for $t=0.02, t=0.39$, and $t=1$ in $1(\mathrm{~d}), 1(\mathrm{e})$, and $1(\mathrm{f})$, respectively. $\Phi(t, \mathbf{x})-|\nabla u(t, \mathbf{x})|$ for $t=0.02, t=0.39$, and $t=1$ in $1(\mathrm{~g}), 1(\mathrm{~h})$, and $1(\mathrm{i})$, respectively. $1(\mathrm{j}):$ Active set at time $t=0.39 .1(\mathrm{k})$ : Active set at time $t=1$.

occurs. The asymptotic behavior of the approximate solution to the QVI, i.e., $u^{\infty}=$ $\lim _{t \rightarrow \infty} u(t)$ seems, in this case, to satisfy the Poisson equation $-\delta \Delta u^{\infty}=r_{1} \varphi(x, y)$.

For each of the time steps within $m \in(1,18)$ (this corresponds to the time interval $0 \leq t \leq 0.18)$, the behavior of the linear convergence coefficient associated with the fixed-point type iteration $\mu^{\ell}:=\left|v^{\ell+2}-v^{\ell+1}\right|_{V} /\left|v^{\ell+1}-v^{\ell}\right|_{V}$, where $v^{\ell}$ is determined in step 5 of Algorithm 1, shows regions of linear convergence as predicted by our theory. As the size of the mesh decreases, the number of iterations for which linear convergence is observed also increases. This is consistent with the described results, 


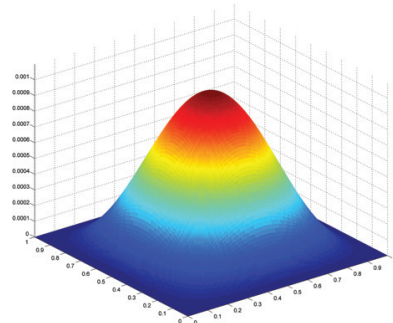

(a)

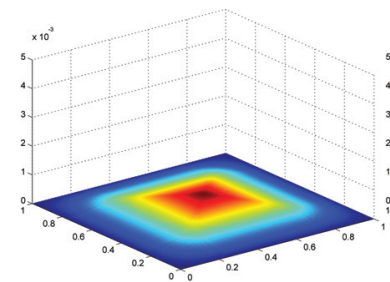

(d)

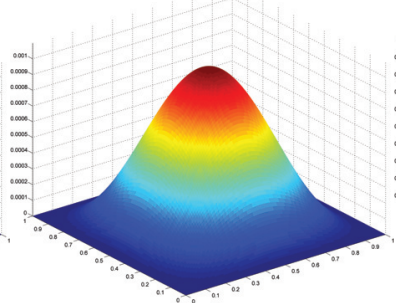

(b)

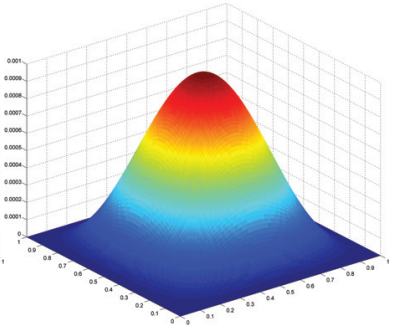

(c)

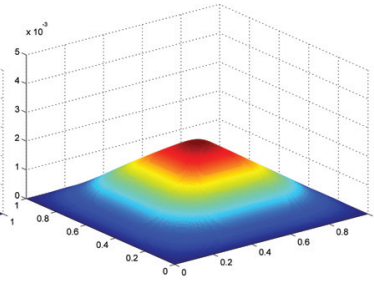

(e)

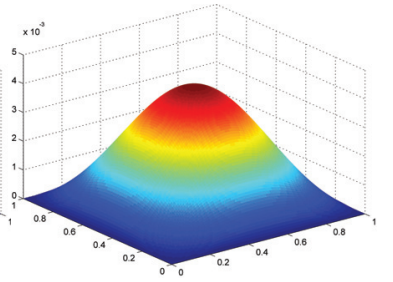

(f)

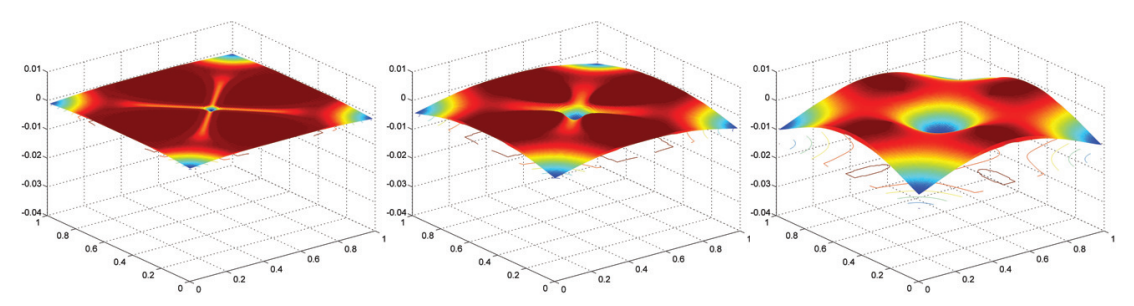

(g)

(h)

(i)

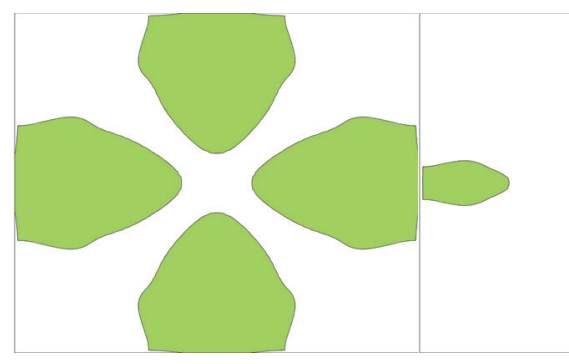

(j)

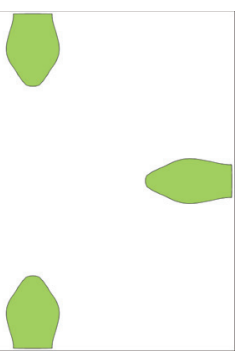

(k)

Fig. 2. The forcing term $t \mapsto f(t, \mathbf{x})$ at $t=0.02,0.10,0.15$ is shown in Figures 2(a), 2(b), and $2(\mathrm{c})$, respectively. $(t, \mathbf{x}) \mapsto u(t, \mathbf{x})$ for $t=0.02, t=0.10$, and $t=0.15$ in $2(\mathrm{~d}), 2(\mathrm{e})$, and $2(\mathrm{f})$, respectively. $\Phi(t, \mathbf{x})-|\nabla u(t, \mathbf{x})|$ for $t=0.02, t=0.10$, and $t=0.15$ in $2(\mathrm{~g}), 2(\mathrm{~h})$, and $2(\mathrm{i})$, respectively. Figure $2(\mathrm{j})$ shows the approximation of the active set at time $t=0.10$ and Figure $2(\mathrm{k})$ at time $t=0.15$.

as linear convergence in each time step is expected when $V=H_{0}^{1}(\Omega)$. For $k=1 / 128$, this is observed up to $\ell=13$. For a larger iteration index, the difference between two consecutive iterations is below $1 \mathrm{e}-9$ and the iteration stagnates.

7.4. Example 3. We consider $p=3$. As in the previous examples, the computational domain consists of $M^{2}$ uniformly distributed nodes on $\Omega=(0,1) \times(0,1)$, 
where $M=128$ and the mesh size is $h=1 /(\mathrm{M}+1)$. The time interval $\mathrm{I}=(0,1)$ consists of a uniform mesh of mesh size $k=1 / 100$. The parameters for the forcing term are $r_{1}=0.1, r_{2}=2, r_{3}=100$, and $r_{4}=0.5$ and the upper bound operator is taken to be

$$
\begin{aligned}
& \Phi(v, t)=\theta(t, \Gamma(v, t)) \eta(\mathbf{x}), \quad \text { where } \\
& \Gamma(v, t)=100 \chi_{\left(r_{4}, 1\right)}(t) \int_{0}^{t}(t-s)^{2} \chi_{\left(r_{4}, 1\right)}(s)\left(\int_{\Omega} v(s, \mathbf{x}) \mathrm{d} \mathbf{x}\right) \mathrm{d} s
\end{aligned}
$$

with $\theta(t, \beta)=0.01\left(1-((\beta+t)(\beta+t-1))^{2}\right)$ and $\eta(x, y)=1+(x y(x-1)(y-1))^{2}$.

The forcing term $t \mapsto f(t, \mathbf{x})$ in this example is zero until $t=0.5$ snapshots at $t=0.52,0.62,0.90$ are shown in Figures 3(a), 3(b), and 3(c), respectively. Since the initial condition for the problem is zero, and zero remains a feasible state, the approximate solution to the parabolic QVI $t \mapsto u(t, \mathbf{x})$ remains zero until $t=0.5$ and then starts to change. Its behavior for $t=0.52,0.62,0.90$ is displayed in Figures 3(d), $3(\mathrm{e})$, and $3(\mathrm{f})$, respectively. It is interesting to observe that the approximate solution at $t=0.90$ in Figure 3(f) does not have flat sides, but instead the values of the norm of the gradient, i.e., $\mathbf{x} \mapsto|\nabla u(0.9, \mathbf{x})|$, become larger the closer $\mathbf{x}$ gets to the center of the unit square. The reason for the latter behavior is due to the fact that the upper bound of the gradient constraint (for a fixed $v \in \mathscr{V}$ and $t$ ) is proportional to $\eta(x, y)=\left(1+(x y(x-1)(y-1))^{2}\right)$ and at $t=0.9$ the solution is active in most of the unit square. The latter can be seen in the approximation of the active set $t \mapsto \mathbb{A}(t)$ in Figure 3(l).

8. Discussion and further research. In Theorem 1, a general scheme for approximation of a solution (and its existence) of $\left(\mathrm{P}_{\mathrm{QVI}}\right)$ is developed. This result is implemented in section 6 with the aid of the results in [19] which state that, under certain hypotheses, the resolution of certain elliptic QVI problems is well posed. For an implementation based on a Euler-type method for discretization in time, the previous also requires a "causal" form of the set $\mathbf{K}(v, t)$, that is, $\mathbf{K}(v, t)$ depends only on the historical values $\{v(s) \in V: s \in[0, t]\}$. This somehow restricts the scope of Theorem 1. On the other hand, there seems to be a clear extension of the results in [19] to parabolic problems, which would allow an application of Theorem 1 to much more general forms of $\mathscr{K}(v)=\{w \in \mathscr{V}: w(t) \in \mathbf{K}(v, t)\}$. This constitutes a primary area of future research.

The resolution of step 5 of Algorithm 1 uses a continuation technique (on the discretized version of the penalized problem (7.4)) and requires a careful selection of the sequence of penalty parameters $\left\{\gamma_{i}\right\}_{i=1}$ for the Newton iterations to converge (for a fixed $\gamma_{i}$ ). The strategy for this has been fully described in [19] and it is determined by three parameters: MinGammaStep, MaxGammaStep, and $\gamma_{t o l}$; the first two control the minimum and the maximum for $\left|\gamma_{i+1}-\gamma_{i}\right|$ with $i \in \mathbb{N}$, and $\gamma_{t o l}$ controls the size of the step adaptively (for a full description, see section 6.2 in [19]). The smaller these three parameters are chosen, the more conservative the continuation technique is. Such a conservative setting is especially required in the case when $p=3$, which implies that the problem is essentially nonlinear due to $A=-\Delta_{p}$, and when the upper bound of the gradient constraint is spatially variant.

In our examples, the comparison among the number of Newton iterations (for each $\gamma$ ) with respect to the (spatial) mesh size $k=1 / 16,1 / 32,1 / 64,1 / 128$ suggests mesh independent behavior of the semismooth Newton solver used for the discretized version of (7.4). In fact, the number of Newton iterations shows little variation with 


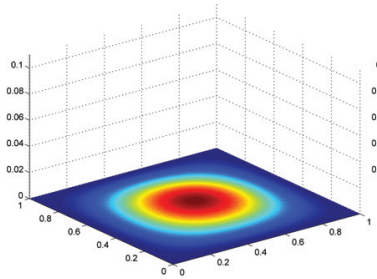

(a)

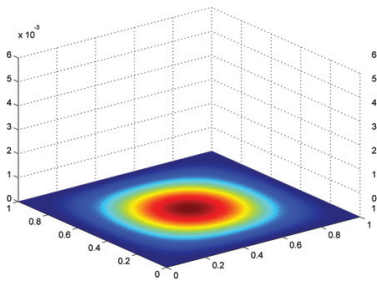

(d)

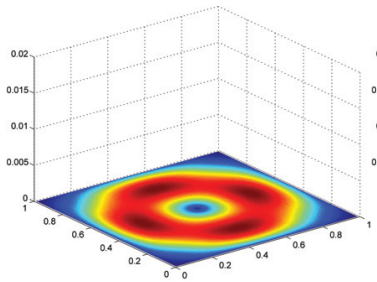

$(\mathrm{g})$

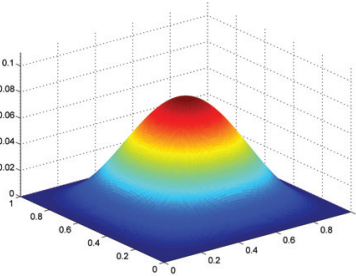

(b)

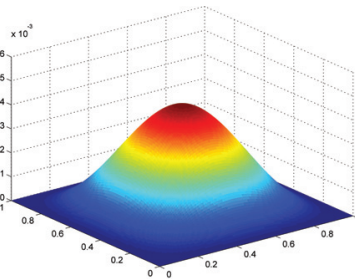

(e)

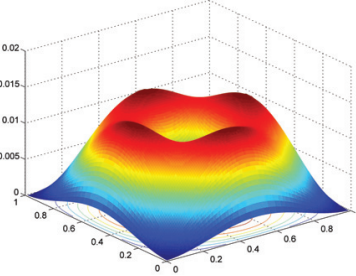

(h)

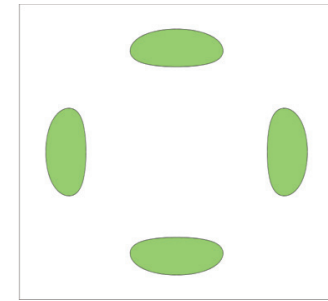

(j)

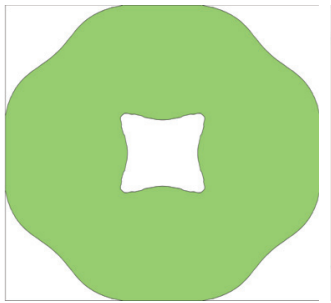

(k)

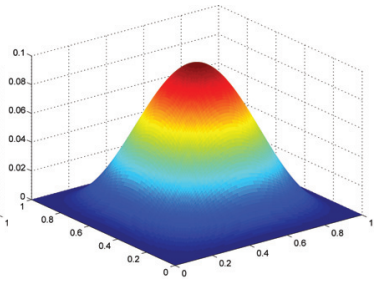

(c)

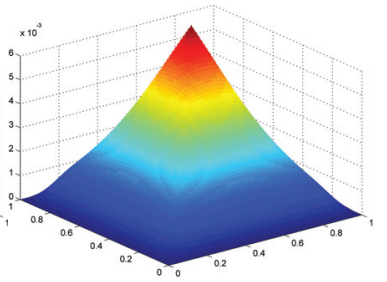

(f)

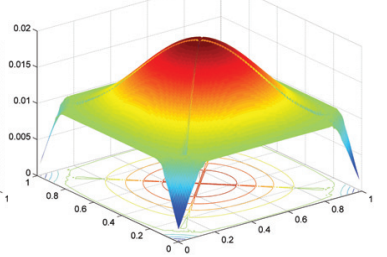

(i)

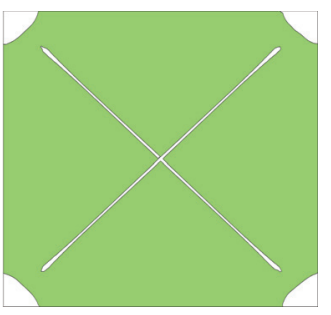

(1)

Fig. 3. Figures $3(\mathrm{a}), 3(\mathrm{~b})$, and 3(c) show the approximation of $(t, \mathbf{x}) \mapsto f(t, \mathbf{x})$ at times $t=$ $0.52,0.62,0.90$, respectively. Figures $3(\mathrm{~d}), 3(\mathrm{e})$, and $3(\mathrm{f})$ show the approximation of $(t, \mathbf{x}) \mapsto u(t, \mathbf{x})$ at times $t=0.52,0.62,0.90$, respectively. Figures $3(\mathrm{~g}), 3(\mathrm{~h})$, and $3(\mathrm{i})$ show the approximation of $(t, \mathbf{x}) \mapsto|\nabla u(t, \mathbf{x})|$ at times $t=0.52,0.62,0.90$, respectively. Figures $3(\mathrm{j}), 3(\mathrm{k})$, and $3(\mathrm{l})$ show the approximation of the active sets at times $t=0.62,0.65,0.90$, respectively.

respect to $k$ and for $\gamma \geq 300$ (in each of the examples, for each $\gamma$ ) this number is below 3. It should be noted that although it can be proven that $F_{\gamma}$ is Newton (or slantly) differentiable, in order to prove that the Newton type iteration converges in function space, it is required to prove that $F_{\gamma}$ has a bounded inverse in some neighborhood of $u_{\gamma}$ such that $F_{\gamma}\left(u_{\gamma}\right)=0$. This latter fact - to the best of our knowledge - has not been proven yet and remains a challenge for future research. 


\section{REFERENCES}

[1] R. A. Adams and J. J. F. Fournier, Sobolev Spaces, 2nd ed., Academic Press, Amsterdam, 2003.

[2] W. Arendt, C. J. K. Batty, M. Hieber, and F. Neubrander, Vector-valued Laplace Transforms and Cauchy Problems, 2nd ed., Birkhäuser, Basel, 2011.

[3] C. Baiocchi And A. CAPelo, Variational and Quasivariational Inequalities, John Wiley \& Sons, New York, 1984.

[4] A. Bensoussan and J.-L. Lions, Controle impulsionnel et inéquations quasi-variationnelles d'évolutions, C. R. Acad. Sci. Paris Sér. A-B, 276 (1974), pp. A1333-A1338.

[5] P. Beremlijski, J. Haslinger, M. KoČvara, and J. Outrata, Shape optimization in contact problems with Coulomb friction, SIAM J. Optim., 13 (2002), pp. 561-587.

[6] H. BERENS, Equivalent representations for the infinitesimal generator of higher orders in semigroup theory, Nederl. Akad. Wetensch. Proc. Ser. A, 27 (1965), pp. 497-512.

[7] H. BRÉZIS, Équations et inéquations non linéaires dans les espaces vectoriels en dualité, Ann. Inst. Fourier, 18 (1968), pp. 115-175.

[8] H. Brézis and C. Bardos, Sur une classe de problèmes d'évolution non linéaires, J. Diff. Equ., 6 (1969), pp. 345-394.

[9] X. Chen, Z. NAShed, AND L. QI, Smoothing methods and semismooth methods for nondifferentiable operator equations, SIAM J. Numer. Anal., 38 (2000), pp. 1200-1216.

[10] Z. Denkowski, S. Migórski, and N. S. Papageorgiou, An Introduction to Nonlinear Analysis: Applications, Kluwer, Boston, 2003.

[11] Z. Ditzian, A new fractional form of the Landau-Kolmogorov inequality, J. Math. Anal. Appl., 148 (1990), pp. 528-537.

[12] G. Duvaut and J.-L. Lions, Les Inéquations en Mécanique et en Physique, Dunod, Paris, 1972.

[13] C. Fefferman and E. Stein, Some maximal inequalities, Amer. J. Math., 93 (1971), pp. 107115.

[14] G. Fichera, Problemi elastotatici con vincoli unilaterali: Il problema di signorini con ambigue condizioni al contorno, Atti. Accad. Naz. Lincei Mem., Mem. Cl. Sci. Fis. Mat. Natur. Sez. I (8), 7 (1964), pp. 91-140.

[15] J. García-Cuerva and J. L. Rubio de Francia, Weighted Norm Inequalities and Related Topics, North-Holland, Amsterdam, 1985.

[16] P. T. HARKer, Generalized Nash games and quasi-variational inequalities, European J. Oper. Res., 54 (1991), pp. 81-94.

[17] E. Hille ANd R. S. Phillips, Functional Analysis and Semi-groups, American Mathematical Society, Providence, RI, 1957.

[18] M. Hintermüller, K. Ito, And K. Kunisch, The primal-dual active set strategy as a semismooth Newton method, SIAM J. Optim., 13 (2002), pp. 865-888.

[19] M. Hintermüller and C. N. Rautenberg, A sequential minimization technique for elliptic quasi-variational inequalities with gradient constraints, SIAM J. Optim., 22 (2012), pp. $1224-1257$.

[20] K. Ito And F. KAppel, Evolution Equations and Approximations, World Scientific, River Edge, NJ, 2002.

[21] C. T. Kelley, Iterative Methods for Linear and Nonlinear Equations, SIAM, Philadelphia, 1995.

[22] D. Kinderlehrer and G. Stampacchia, An Introduction to Variational Inequalities and Their Applications, SIAM, Philadelphia, 2000.

[23] A. S. Kravchuk and P. J. NeittaanmäKI, Variational and Quasi-variational Inequalities in Mechanics, Springer, Dordrecht, 2007.

[24] M. Kunze And J.F. Rodrigues, An elliptic quasi-variational inequality with gradient constraints and some of its applications, Math. Methods Appl. Sci., 23 (2000), pp. 897-908.

[25] J.-L. Lions, Quelques Méthodes de Résolutions des Problémes aux Limites non Linéaires, Dunod, Gauthier-Villars, Paris, 1969.

[26] J.-L. Lions, Sur le côntrole optimal des systemes distribuées, Enseignement Math. (2), 19 (1973), pp. 125-166.

[27] J.-L. Lions, Asymptotic behaviour of solutions of variational inequalitites with highly oscillating coefficients, Applications of Methods of Functional Analysis to Problems in Mechanics, Proc. Joint Symp. IUTAM/IMU. Lecture Notes in Mathematics, Springer, Berlin, 503 (1975).

[28] J.-L. Lions and G. Stampacchia, Variational inequalities, Comm. Pure Appl. Math., 20 (1967), pp. 493-519.

Copyright (C) by SIAM. Unauthorized reproduction of this article is prohibited. 
[29] U. Mosco, Convergence of convex sets and of solutions of variational inequalities, Advances in Math., 3 (1969), pp. 510-585.

[30] J.-S. PAng And M. Fukushima, Quasi-variational inequalities, generalized Nash equilibria, and multi-leader-follower games, Comput. Manag. Sci., 6 (2009), pp. 373-375.

[31] A. PAZY, Semigroups of Linear Opertors and Applications to Partial Differential Equations, Springer-Verlag, New York, 1983.

[32] L. Prigozhin, On the Bean critical-state model in superconductivity, European J. Appl. Math., 7 (1996), pp. 237-247.

[33] L. QI AND D. Sun, A survey of some nonsmooth equations and smoothing Newton methods, in Progress in Optimization, Appl. Optim. 30, Kluwer, Dordrecht, 1969, pp. 121-146.

[34] J.-F. Rodrigues, Obstacle Problems in Mathematical Physics, North-Holland, Amsterdam, 1987.

[35] J.-F. Rodrigues and L. Santos, A parabolic quasi-variational inequality arising in a superconductivity model, Ann. Scuola Norm. Sup. Pisa Cl. Sci. (4), 29 (2000), pp. 153-169.

[36] L. Santos, Variational problems with non-constant gradient constraints, Port. Math. (N.S.), 59 (2002), pp. 205-248.

[37] R. E. Showalter, Monotone Operators in Banach Space and Nonlinear Partial Differential Equations, American Mathematical Society, Providence, RI, 1997.

[38] E. ZeIDler, Nonlinear Functional Analysis and Applications, Vol. II/B: Nonlinear Monotone Operators, Springer-Verlag, New York, 1990.

Copyright $@$ by SIAM. Unauthorized reproduction of this article is prohibited. 